\title{
Minimal composite Higgs models at the LHC
}

\author{
Marcela Carena, ${ }^{a, b, c}$ Leandro Da Rold ${ }^{d}$ and Eduardo Pontón ${ }^{e}$ \\ ${ }^{a}$ Fermi National Accelerator Laboratory, \\ P.O. Box 500, Batavia, IL 60510, U.S.A. \\ ${ }^{b}$ Enrico Fermi Institute, University of Chicago, \\ Chicago, IL 6063\%, U.S.A. \\ ${ }^{c}$ Kavli Institute for Cosmological Physics, University of Chicago, \\ Chicago, IL 60637, U.S.A. \\ ${ }^{d}$ CONICET, Centro Atómico Bariloche and Instituto Balseiro, \\ Av. Bustillo 9500, 8400, S. C. de Bariloche, Argentina \\ e ICTP South American Institute for Fundamental Research and Instituto de Física Teórica, \\ Universidade Estadual Paulista (UNESP), \\ Rua Dr. Bento Teobaldo Ferraz 271, 01140-070, São Paulo, SP Brazil \\ E-mail: carena@fnal.gov, daroldl@cab.cnea.gov.ar, eponton@ift.unesp.br
}

ABstract: We consider composite Higgs models where the Higgs is a pseudo-Nambu Goldstone boson arising from the spontaneous breaking of an approximate global symmetry by some underlying strong dynamics. We focus on the $\mathrm{SO}(5) \rightarrow \mathrm{SO}(4)$ symmetry breaking pattern, assuming the "partial compositeness" paradigm. We study the consequences on Higgs physics of the fermionic representations produced by the strong dynamics, that mix with the Standard Model (SM) degrees of freedom. We consider models based on the lowest-dimensional representations of $\mathrm{SO}(5)$ that allow for the custodial protection of the $Z \bar{b} b$ coupling, i.e. the 5, 10 and 14. We find a generic suppression of the gluon fusion process, while the Higgs branching fractions can be enhanced or suppressed compared to the SM. Interestingly, a precise measurement of the Higgs boson couplings can distinguish between different realizations in the fermionic sector, thus providing crucial information about the nature of the UV dynamics.

Keywords: Higgs Physics, Spontaneous Symmetry Breaking, Beyond Standard Model, Global Symmetries

ARXIV EPRINT: 1402.2987 


\section{Contents}

1 Introduction 1

2 A minimal pNGB Higgs 3

2.1 Effective description: 2-site model 5

2.2 Bosonic sector 6

2.3 Fermionic sector 9

$\begin{array}{lll}2.4 & \text { The low-energy effective theory } & 10\end{array}$

3 Models based on the 1, 5, 10 and 14 reps. of $\mathrm{SO}(5) \quad 12$

$\begin{array}{lll}3.1 & \mathrm{MCHM}_{5} & 14\end{array}$

$\begin{array}{lll}3.2 & \mathrm{MCHM}_{10} & 15\end{array}$

$\begin{array}{lll}3.3 \mathrm{MCHM}_{10-5-10} & 16\end{array}$

$\begin{array}{ll}3.4 \mathrm{MCHM}_{5-5-10} & 17\end{array}$

$\begin{array}{ll}3.5 \mathrm{MCHM}_{5-10-10} & 18\end{array}$

$\begin{array}{lll}3.6 & \mathrm{MCHM}_{14-14-10} & 19\end{array}$

$3.7 \mathrm{MCHM}_{14-1-10} 20$

3.8 Other models based on the lowest-dimensional reps. of $\mathrm{SO}(5) \quad 21$

4 Corrections to low-energy observables in the MCHM 22

5 Higgs potential $\quad \mathbf{2 6}$

5.1 Finiteness of the 1-loop Higgs potential 26

6 Higgs phenomenology 28

$\begin{array}{lll}6.1 & \text { Numerical scan } & 29\end{array}$

6.2 Corrections to the gauge and Yukawa couplings 32

6.3 Higgs production and decay 34

$\begin{array}{lll}6.4 h \rightarrow Z \gamma & 39\end{array}$

$\begin{array}{lll}7 & \text { Tuning in the MCHM } & 41\end{array}$

8 Conclusions $\quad 43$

A Representations of SO(5) 45

$\begin{array}{ll}\text { B Bosonic mass matrices } & 47\end{array}$

$\begin{array}{ll}\text { C Correlators } & \mathbf{4 8}\end{array}$

$\begin{array}{lll}\text { C.1 } & \mathrm{MCHM}_{5} & 48\end{array}$

$\begin{array}{lll}\text { C.2 } & \mathrm{MCHM}_{10} & 49\end{array}$

$\begin{array}{ll}\text { C.3 } \text { MCHM }_{10-5-10} & 49\end{array}$

$\begin{array}{ll}\text { C.4 } \mathrm{MCHM}_{5-5-10} & 49\end{array}$ 
$\begin{array}{lll}\text { C.5 } \text { MCHM }_{5-10-10} & 49\end{array}$

$\begin{array}{ll}\text { C. } 6 \mathrm{MCHM}_{14-1-10} & 50\end{array}$

$\begin{array}{lll}\text { C.7 } & \mathrm{MCHM}_{14-14-10} & 50\end{array}$

$\begin{array}{ll}\text { D Loop-level processes } & 50\end{array}$

\section{Introduction}

The discovery of a SM-like Higgs boson with a mass of about $126 \mathrm{GeV}[1,2]$ represents a fundamental step towards a better understanding of the origin of Electroweak Symmetry Breaking (EWSB). Measuring its couplings with higher precision will be one of the priorities in the $14 \mathrm{TeV}$ run of the $\mathrm{LHC}$, and is one of the main motivations for building a future lepton collider. The phenomenological description of EWSB within the SM framework provides a benchmark against which any deviations in the Higgs boson couplings should be compared, as such deviations could contain the key to a more fundamental understanding of this phenomenon.

A currently open question is whether this particle is elementary (i.e. pointlike), down to distance scales much shorter than the EW scale, or if, on the contrary, it is a composite bound state of more fundamental degrees of freedom, whose physics should be revealed at energies not far above the weak scale. In either case the discovery of this scalar particle is truly remarkable. If it turns out to be elementary it would be the first and only known example of this kind in nature. Its existence at energies low compared to e.g. the Planck scale could indicate that the universe as we know it results from a rather perplexing finetuning, or perhaps more plausibly that there is a symmetry at work as exemplified by supersymmetric scenarios. If it turns out that the Higgs boson is a composite state arising from some underlying strong dynamics, we would be in a situation that also presents new characteristics compared to other known composite scalars. For instance, unlike the pions of QCD, the dynamics of the Higgs boson must lead to EWSB by generating a non-vanishing vacuum expectation value (vev) for the composite scalar.

The fact that the LHC has not observed any major deviation from the SM in its 7-8 TeV run indicates that any new physics should be roughly above $1 \mathrm{TeV}$ (although one can think of specific examples that are less constrained, and also examples that are significantly more constrained). In the context of Higgs compositeness, this means that there must exist a scalar resonance much lighter than the other strong resonances. It is then natural to interpret the Higgs as a pseudo-Nambu Goldstone boson (pNGB) arising from the spontaneous breaking of an approximate global symmetry of the new strong sector [3-7]. This idea has received considerable attention lately [8]. A question of special importance centers on the type of deviations in the Higgs properties that would be expected in such scenarios. This has been studied to some extent within specific realizations of a Higgs as a pNGB, and also in the context of an effective low-energy parametrization such as the SILH [9] and similar approaches [10-16]. 
We will focus here on the minimal case ${ }^{1}$ based on the $\mathrm{SO}(5) \rightarrow \mathrm{SO}(4)$ symmetry breaking pattern [18], which leads to exactly four Nambu-Goldstone bosons and contains a custodial symmetry that ensures that the corrections to certain electroweak observables are sufficiently suppressed. Although the embedding of the SM gauge sector is fixed by the above assumption, there is still a considerable arbitrariness in how the SM fermionic sector is embedded into the framework. This depends, in particular, on which $\mathrm{SO}(5)$ representations for the fermionic resonances are generated by the strong dynamics and would therefore be sensitive to further details of the specific UV realization of the idea. Our aim is to study in detail the implications for the properties of the Higgs boson. In particular, we will show that if one were to measure a robust deviation from the SM in the rates $h \rightarrow \gamma \gamma, h \rightarrow Z Z$ and $h \rightarrow Z \gamma$ and to a lesser extent in $h \rightarrow \tau \tau$, one could gather indirect information regarding the quantum numbers of the fermionic resonances. One also expects a generic reduction of the Higgs production cross section (in particular through gluon fusion), as well as a suppression of all Yukawa couplings w.r.t. the SM.

There have been a number of studies on the phenomenology of a pNGB Higgs as well as partial compositeness. Since the pioneer work of ref. [19] studying Higgs production by gluon fusion, many works have considered the deviations of the Higgs couplings in this setup (i.e. for a pNGB Higgs), exploring the dependence on the degree of compositeness of the fermions, the scale of compositeness and their relation with the spectrum of resonances, among other important variables [20-22]. However most of them have considered generic regions of the parameter space, that could be unphysical, in the sense that either there is no EWSB, or the decay constant of the Higgs and its vev are not separated enough to guarantee compatibility with EW precision measurements, or the spectrum of the lightest level of states does not reproduce the SM one, to cite a few examples. To ensure that these conditions are satisfied and therefore make a realistic study of the Higgs phenomenology, in general requires a full study of the Higgs potential that can only be performed in a well defined model, with the risk of loosing some generality. One of the purposes of this work is to make a step in that direction. We consider a family of well defined models, with the same pattern of symmetry breaking for the pNGB Higgs but allowing different representations for the fields of the theory. This still represents considerable freedom and for this reason we make some restrictive assumptions that ensure calculability of the Higgs potential within the framework of a two site model. We will also assume that at high energies the symmetry behind the pNGB is linearly realized for the massive resonances, and for that reason we will include massive resonances in complete $\mathrm{SO}(5)$ representations. It is possible to relax some of these assumptions, for example by considering models with more sites, by allowing for non-linear realizations, or even by allowing for logarithmic divergences of the potential. ${ }^{2}$ Nevertheless, we hope that our setup can still capture generic features of minimal pNGB

\footnotetext{
${ }^{1}$ The terminology "Minimal Composite Higgs Model (MCHM)" was actually introduced in a slightly different context in [17]. Our study is limited to more recent models based on the pNGB idea which have also been named MCHM [18]. Since we consider a variety of fermionic realizations, here the "minimality" refers specifically to the (common) bosonic sector.

${ }^{2}$ L.D. thanks Gilad Perez for discussions on this topic.
} 
models. ${ }^{3}$ We will show that it can give information on the size of the corrections that one can expect on the Higgs phenomenology as well as on the wealth and direction of corrections that follow by allowing for different representations of the fields.

This paper is organized as follows. In section 2 we review the basic aspects of the effective two-site description of the composite Higgs scenario. In section 3 we present the details of the specific models we study in this work, which differ in the realization of the fermionic sector. In section 4 we describe the low-energy consequences of the pNGB nature of the Higgs and the presence of the composite resonances, while in section 5 we discuss the properties of the Higgs potential. Section 6 contains our numerical results, while section 7 contains some remarks on the tuning of the phenomenologically viable models. We summarize and conclude in section 8. We also include four appendices: appendix A summarizes several useful group theoretical results, appendix B contains the mass matrices of the gauge sector of the models, appendix $\mathrm{C}$ contains all the correlators for the low-energy limit of the various models, and finally appendix D summarizes how we compute the 1-loop processes $h \rightarrow \gamma \gamma, h \rightarrow Z Z$ and $h \rightarrow Z \gamma$.

\section{A minimal pNGB Higgs}

We are interested in the minimal model that can deliver the Higgs as a pNGB resonance arising from the spontaneous breaking of a global symmetry in a strongly coupled sector (SCFT). We will assume that the SCFT has an exact global symmetry that is spontaneously broken to a subgroup by effects of the strong dynamics, with the Higgs being the associated Nambu-Goldstone boson (NGB). The interactions of the fields in the SCFT with the SM fields explicitly break the global symmetry, leading to a Higgs potential at loop level. In this case the degeneracy of the vacuum is uplifted and the Higgs becomes a pNGB, leading to a natural separation between the scale of the resonances and the Higgs mass. Usually the gauge contributions to the 1-loop Coleman-Weinberg potential are aligned with the EW gauge group. However the fermion contributions, that are expected to be large because of the large top mass, can induce a missalignement of the vacuum triggering EW symmetry breaking dynamically.

Ref. [18] has shown that the minimal group containing the SM EW gauge symmetry and an unbroken custodial symmetry that can lead to a pNGB Higgs is $\mathrm{SO}(5)$. This group is spontaneously broken to $\mathrm{SO}(4) \simeq \mathrm{SU}(2)_{L} \times \mathrm{SU}(2)_{R}$, with the Higgs being the NGB in the coset $\mathrm{SO}(5) / \mathrm{SO}(4)$ that transforms as a 4 of $\mathrm{SO}(4)$. Besides the Higgs, the SCFT is assumed to lead to vector resonances in the adjoint representation of the global group (these are created by the Noether currents of this symmetry). In addition, one assumes the existence of fermion resonances, some of which can mix with the SM degrees of freedom. We will consider that all the massive composite resonances are in complete irreducible representations of $\mathrm{SO}(5)$, realizing the symmetry in a linear way. All the composite states are taken to interact with typical couplings $g_{\rho} \gg g_{S M}$. The SM gauge and fermion fields

\footnotetext{
${ }^{3}$ Recently, another class of pNGB models based on four-fermion interactions has been discussed in [23]. Although they rely on a different breaking pattern, in principle they could be extended to $\mathrm{SO}(5) / \mathrm{SO}(4)$, following the analysis of [24].
} 
can be considered as external sources probing the SCFT, i.e.: elementary fields. The SM particles do not interact with the Higgs at leading order, but these interactions are mediated by the resonances of the SCFT that mix with the elementary fields.

The gauge fields of the SM weakly gauge a subgroup of the SCFT global symmetry. The conserved currents of the SCFT associated to this subgroup couple linearly with the SM gauge fields, explicitly breaking the global symmetry. The masses of the EW vector bosons arises from mixing between the vector resonances created by the SCFT currents and the SM gauge fields, as well as from the Higgs interactions.

We are also interested in partial compositeness of the SM fermions, that can be realized if the elementary fermions couple linearly with operators of the SCFT: $\mathcal{L} \supset \lambda \bar{\psi} \mathcal{O}_{\psi}$. The low energy scaling of the coupling $\lambda$ is controlled by the dimension of the corresponding SCFT operator $D=\operatorname{dim}\left[\mathcal{O}_{\psi}\right][18,25]$. For $D>5 / 2$ the coupling is irrelevant leading to small mixing between the elementary fermions and the fermionic resonances created by the SCFT operator. For $D<5 / 2$ the coupling is relevant leading to large mixing between the elementary fermion and the resonances, and thus to a large Yukawa coupling. The former case leads to light states that are mainly elementary, whereas the latter one can lead to large fermion masses, as for the top quark, which is associated with a large degree of compositeness.

The proper normalization of hypercharge for fermions requires the introduction of an extra $\mathrm{U}(1)_{X}$ symmetry in the composite sector, with the identification $Y=T_{R}^{3}+X$, where $T_{R}^{3}$ is the diagonal generator of $\mathrm{SU}(2)_{R}$. The $\mathrm{SU}(2)_{R}$ charge of the composite operators $\mathcal{O}_{\psi}$ is not fixed, allowing for different representations $\mathbf{r}_{\mathcal{O}}$ under $\mathrm{SO}(5)$. However, the stringent constraints on the corrections to the $Z b \bar{b}$ couplings arising from LEP and SLC require a nontrivial protection of the $Z b_{L} \bar{b}_{L}$ coupling. Ref. [26] has shown that there is a subgroup of the custodial symmetry $\mathrm{O}(3)$ that can ensure that the corrections to this coupling are indeed sufficiently suppressed. This symmetry requires that the representation $\mathbf{r}_{\mathcal{O}_{q}}$, where $\mathcal{O}_{q}$ is coupled to the doublet of the third generation $q_{L}$, decompose under $\mathrm{SO}(4)$ as: $\mathbf{r}_{\mathcal{O}_{q}} \simeq \mathbf{4} \oplus \ldots$ The smallest representations satisfying this condition are: $\mathbf{r}=\mathbf{5 , 1 0}, \mathbf{1 4}$. On the other hand, invariance of the SCFT under $\mathrm{SO}(5) \times \mathrm{U}(1)_{X}$ restricts the representations of the operators $\mathcal{O}_{u}$ and $\mathcal{O}_{d}$, coupled with $t_{R}$ and $b_{R}$ respectively. In this work we will consider several representations $\mathbf{r}_{\mathcal{O}}$ subject to the above restrictions, and we will study their impact in the Higgs phenomenology at the LHC.

We also note that, although a generic strongly coupled theory is expected to yield resonances with spins other than $1 / 2$ and 1 , the contributions of such states to the lowenergy physics are expected to be small. This is mainly because they cannot mix with the SM fields, which as we will see leads to the dominant deviations from the SM. Also, as illustated by our analysis of the direct impact of the heavy spin-1 resonances, all such resonances are expected to be relatively heavy and lead to suppressed effects. The fermionic resonances are special in that one often finds parametrically lighter states that can give a non-negligible contribution (apart from the effects due to mixing). Thus, we will be content with including only the spin-1 (which are common to all the models we consider) and spin- $1 / 2$ resonances in our anaysis.

The scenario described in the previous paragraphs can be realized by considering a theory in a slice of a warped five dimensional space-time, with the metric being $\mathrm{AdS}_{5}$ near 
the UV. The elementary fields and resonances can be identified with degrees of freedom on the UV boundary and Kaluza-Klein states, respectively. However it is possible to capture most of the essential ingredients by considering a theory with the first level of resonances only, as in the elementary/composite description of ref. [27]. At low energies one considers an effective description with elementary fields, one level of resonances and linear mixing between them. This description has more freedom than the full 5D theory, allowing for new terms [28] as well as a lack of correlation between some parameters, such as the masses of the different resonances. It also has a cut-off of order a few TeV. However it is able to parametrize a family of realistic theories with a pNGB Higgs and it is still predictive enough to explore, at the LHC, the consequences of the symmetries protecting the Higgs potential. In the next subsections we will summarize a realization of this effective theory.

\subsection{Effective description: 2-site model}

We consider the effective description of the Higgs as a pNGB arising from a strongly coupled sector, as introduced in ref. [28] (see also [29]). The simplest model has two sites: one called site- 0 that describes elementary fields, and another called site- 1 describing the first level of resonances arising from the strongly coupled sector (the composite sector). Site-0 contains a set of gauge and fermion fields with the same symmetry group and fermionic representations as the SM. We will call $G_{0}$ the gauge symmetry of this site: $G_{0}=\mathrm{SU}(2)_{L} \times \mathrm{U}(1)_{Y}{ }^{4}$ Note that there are no elementary scalar fields. On site- 1 we consider a gauge symmetry $G_{1}=\mathrm{SO}(5) \times \mathrm{U}(1)_{X}$, which allows to describe effectively the lowest lying spin-1 resonances of the strong dynamics. Site-1 also contains several multiplets of fermion fields in various representations of $G_{1}$, which will be described in detail later. The two sites are connected by a $\sigma$-model field $\Omega,{ }^{5}$ transforming as $\Omega \rightarrow g_{0} \Omega g_{1}^{\dagger}$, with $g_{0,1} \in G_{0,1}$. In figure 1 we show the Moose diagram corresponding to this theory. We use lower case letters for fields on site- 0 and upper case letters for fields on site- 1.

It turns out to be very convenient to extend $G_{0}$ to a spurious $G_{0}^{\prime}=\mathrm{SO}(5) \times \mathrm{U}(1)_{x}$. This is achieved by introducing non-dynamical gauge and fermion fields on site- 0 that, together with the dynamical fields that fill representation of $G_{0} \subset G_{0}^{\prime}$, complete full representations of $G_{0}^{\prime}$. When one considers all the fields on site- 0 as non-dynamical, they act as sources for an exact global $G_{0}^{\prime}$ symmetry, which is to be thought as a global symmetry of the strongly coupled sector. We assume that the strong dynamics giving rise to the composite resonances spontaneously breaks the $\mathrm{SO}(5)$ global factor down to $\mathrm{SO}(4)$, thus delivering a set of NGB's in the coset $\mathrm{SO}(5) / \mathrm{SO}(4)$. These will be identified as the composite Higgs, and are described by a field $\Phi_{1}$ as shown in figure 1 . The presence of the dynamical fields on site-0 explicitly breaks $G_{0}^{\prime}$ (e.g. by their kinetic terms, which are not present for the spurious fields on site-0), and therefore generates a potential for the Higgs, which becomes a pNGB. This potential is often calculable and is one of the attractive theoretical features of these scenarios. The observation of a Higgs boson at the LHC and the measurement of its mass and couplings then imposes non-trivial constraints on the parameters of the model.

\footnotetext{
${ }^{4}$ There is also a color $\mathrm{SU}(3)_{C}$ on each site, but we omit mentioning these factors in the following.

${ }^{5}$ Strictly speaking, there are two link fields, $\Omega$ and $\Omega_{X}$, for the $\mathrm{SO}(5)$ and $\mathrm{U}(1)_{X}$ factors. These will be described in detail below.
} 


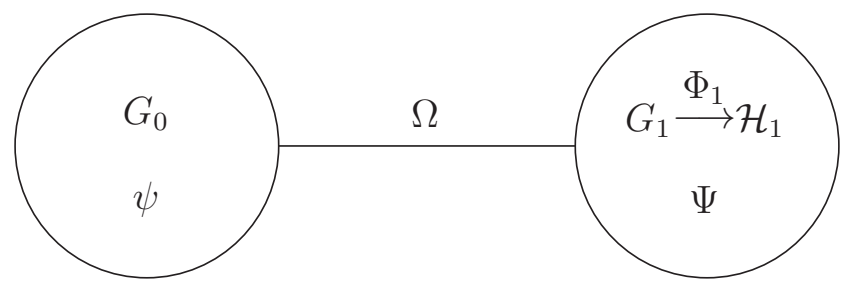

Figure 1. Moose diagram of the two site theory describing the model. $G_{0}$ is the SM gauge symmetry and $G_{1}=\mathrm{SO}(5) \times \mathrm{U}(1)_{X}$. The (spontaneous) breaking of $G_{1}$ down to $\mathcal{H}_{1}=\mathrm{SO}(4) \times \mathrm{U}(1)_{X}$, is parametrized by a field $\Phi_{1}$, that transforms under $G_{1}$ as a 5 of $\operatorname{SO}(5)$ with $Q_{X}=0$, and whose vev is $\left\langle\Phi_{1}\right\rangle=\{0,0,0,0,1\}^{T}$. The link field transforms like $\Omega \rightarrow g_{0} \Omega g_{1}^{\dagger}$, with $g_{0,1} \in G_{0,1}$.

As we will see in detail below, the presence of $\Omega$ allows to realize partial compositeness of the fermions through bilinear terms involving a fermion $\psi$ at site- 0 and a fermion $\Psi$ at site-1. It also leads to non-zero masses for the axial combination of the gauge fields in sites- 0 and 1 , and contains the would-be NGB's that are eaten in this process.

\subsection{Bosonic sector}

Let us consider first the Lagrangian describing the fields that parametrize the $\mathrm{SO}(5) \rightarrow$ $\mathrm{SO}(4)$ breaking. ${ }^{6}$ We denote the unbroken generators of $\mathrm{SO}(5)$ [i.e. of $\mathcal{H}_{1} \equiv S O(4) \simeq$ $\left.\mathrm{SU}(2)_{L} \times \mathrm{SU}(2)_{R}\right]$ by $T^{a}$, while the broken ones are denoted by $T^{\hat{a}}$. For reference, we give their explicit expressions in a convenient basis in appendix A. The NGB's are parametrized by

$$
U(\Pi)=e^{i \Pi / f_{1}}, \quad \Pi=\Pi^{\hat{a}} T^{\hat{a}},
$$

where $f_{1}$ is the corresponding decay constant. The $G_{1}=\mathrm{SO}(5)$ symmetry is non-linearly realized, that is, under a $g_{1} \in G_{1}$ we have $U \rightarrow g_{1} U h_{1}\left(g_{1} ; \Pi\right)^{\dagger}$, where $h_{1}\left(g_{1} ; \Pi\right) \in \mathcal{H}_{1}$ is an element of the unbroken group, that depends on the $\mathrm{SO}(5)$ transformation $g_{1}$ and the NGB fields $\Pi$. The leading order Lagrangian of these NGB's is

$$
\mathcal{L}_{\mathrm{NGB}}=\frac{f_{1}^{2}}{2} \mathcal{D}_{\mu}^{\hat{a}} \mathcal{D}^{\mu \hat{a}}
$$

with $\mathcal{D}_{\mu}^{\hat{a}}$ implicitly defined by $U^{\dagger} D_{\mu} U=i \mathcal{E}_{\mu}^{a} T^{a}+\mathcal{D}_{\mu}^{\hat{a}} T^{\hat{a}}$. The covariant derivative contains the composite spin-1 resonances, $A_{\mu}$, and leads to the interactions between these and the NGB's. We defer the description of the interactions between the NGB's and the fermions $\Psi$ on site-1 to the next section.

One can obtain a simpler and more explicit description of the above sector by defining $\Phi_{1}=U \phi_{1}$, with $\phi_{1}^{B}=\delta^{B 5}(B=1, \ldots 5)$. Under a $g_{1} \in G_{1}$ one simply has $\Phi_{1} \rightarrow g_{1} \Phi_{1}$, and it can be checked that the above Lagrangian can be written as

$$
\mathcal{L}_{\mathrm{NGB}}=\frac{f_{1}^{2}}{2}\left|D_{\mu} \Phi_{1}\right|^{2}
$$

\footnotetext{
${ }^{6}$ Since the NGB fields are neutral under $\mathrm{U}(1)_{X}$, we omit this factor for simplicity in this discussion, but it should be understood.
} 
In this form, the breaking of $\mathrm{SO}(5)$ down to $\mathrm{SO}(4)$ is simply parametrized by $\left\langle\Phi_{1}\right\rangle=$ $\{0,0,0,0,1\}^{T}$.

As for the Lagrangian describing the $\sigma$-model connecting the two sites, at leading order one has:

$$
\mathcal{L}_{\Omega}=\frac{f_{\Omega}^{2}}{4} \operatorname{tr}\left|D_{\mu} \Omega\right|^{2}+\frac{f_{\Omega_{X}}^{2}}{4}\left|D_{\mu} \Omega_{X}\right|^{2}
$$

with

$$
\Omega=e^{\sqrt{2} i \Pi_{\Omega} / f_{\Omega}}, \quad \quad \Omega_{X}=e^{\sqrt{2} i \Pi_{\Omega_{X}} / f_{\Omega_{X}}},
$$

where $\Pi_{\Omega}=\Pi_{\Omega}^{b} T_{0-1}^{b}$ and $T_{0-1}^{b}$ denote the generators of $\mathrm{SO}(5)_{0} \times \mathrm{SO}(5)_{1} / \mathrm{SO}(5)_{0+1}$, with $\mathrm{SO}(5)_{0+1}$ denoting the diagonal (vector) subgroup of $\mathrm{SO}(5)_{0} \times \mathrm{SO}(5)_{1}$. We have also included an additional link field $\Omega_{X}$ (with its decay constant $f_{\Omega_{X}}$ and charges $Q_{x}=Q_{X}=$ 1) for the $\mathrm{U}(1)_{x} \times \mathrm{U}(1)_{X}$ factors. The covariant derivatives above are given by

$$
D_{\mu} \Omega=\partial_{\mu} \Omega-i \tilde{a}_{\mu} \Omega+i \Omega \tilde{A}_{\mu}, \quad D_{\mu} \Omega_{X}=\partial_{\mu} \Omega_{X}-i \tilde{x}_{\mu} \Omega_{X}+i \Omega_{X} \tilde{X}_{\mu},
$$

where $\left\{\tilde{a}_{\mu}, \tilde{x}_{\mu}\right\}$ and $\left\{\tilde{A}_{\mu}, \tilde{X}_{\mu}\right\}$ are the gauge fields of site- 0 and site- 1 , respectively (the tildes denote non-canonical normalization).

Besides the terms above the bosonic Lagrangian includes the kinetic terms for the gauge fields of $G_{0}$ and $G_{1}$ :

$$
\mathcal{L}_{\text {gauge }}=-\frac{1}{4 g_{0}^{2}} \tilde{w}_{L \mu \nu}^{j} \tilde{w}_{L}^{j \mu \nu}-\frac{1}{4 g_{0}^{\prime 2}} \tilde{b}_{\mu \nu} \tilde{b}^{\mu \nu}-\frac{1}{4 g_{\rho}^{2}} \tilde{A}_{\mu \nu}^{B} \tilde{A}^{B \mu \nu}-\frac{1}{4 g_{X}^{2}} \tilde{X}_{\mu \nu} \tilde{X}^{\mu \nu},
$$

where $j=1,2,3, B=1, \ldots, 10$, and $\tilde{w}_{L \mu \nu}^{j}, \tilde{b}_{\mu \nu}$ and $\left\{\tilde{A}_{\mu \nu}^{B}, \tilde{X}_{\mu \nu}\right\}$ are the field strengths of $\mathrm{SU}(2)_{L}, \mathrm{U}(1)_{Y}$ and $\mathrm{SO}(5) \times \mathrm{U}(1)_{X}$, respectively. The embedding of $\mathrm{U}(1)_{Y} \subset \mathrm{SU}(2)_{R} \times \mathrm{U}(1)_{x}$ on site- 0 is obtained by the identifications $\tilde{w}_{R \mu}^{3}=\tilde{x}_{\mu}=\tilde{b}_{\mu}$ so that $b_{\mu}$ couples to $Y=$ $T_{R}^{3}+Q_{X}$ with coupling $g_{0}^{\prime}=g_{0} g_{x} / \sqrt{g_{0}^{2}+g_{x}^{2}} \cdot$. The relation between the couplings $g_{0}$ and $g_{0}^{\prime}$ and their SM counterparts will be specified below, and similarly for the relation between the elementary gauge fields $\tilde{w}_{L}^{\mu}$ and $\tilde{b}^{\mu}$, and the SM gauge fields $W_{L}^{\mu}$ and $B^{\mu}$. We assume that the couplings characterizing the interactions of the composite spin-1 fields, $g_{\rho}$ and $g_{X}$, are large but still perturbative.

The physical field content of the theory becomes evident in unitary gauge, where the would-be NGB's eaten by the composite $A_{\mu}$ 's are set to zero. This is achieved by a gauge transformation $g_{1}=\Omega$ (and using $\Omega_{X}$ for the $\mathrm{U}(1)_{X}$ factor). The physical NGB's are then fully parametrized by

$$
\Phi \equiv \Omega \Phi_{1}=\frac{1}{h} \sin \frac{h}{f_{h}}\left(h_{1}, h_{2}, h_{3}, h_{4}, h \cot \frac{h}{f_{h}}\right)^{T},
$$

with

$$
\frac{1}{f_{h}^{2}}=\frac{1}{f_{\Omega}^{2}}+\frac{1}{f_{1}^{2}}, \quad h^{2}=\sum_{a} h^{\hat{a}} h^{\hat{a}}
$$

\footnotetext{
${ }^{7}$ Here the fields are normalized according to $\mathcal{L}_{\text {gauge }} \supset-1 /\left(4 g_{0}^{2}\right) \tilde{w}_{R \mu \nu}^{3} \tilde{w}_{R}^{3 \mu \nu}-1 /\left(4 g_{x}^{2}\right) \tilde{x}_{\mu \nu} \tilde{x}^{\mu \nu}$, while $\tilde{b}_{\mu}$ is normalized as in eq. (2.7).
} 
The vacuum is characterized by the variable $\epsilon=\sin \left(v / f_{h}\right)$, with $v=\langle h\rangle$ and $\langle\Phi\rangle^{T}=$ $\left(0,0,0, \epsilon, \sqrt{1-\epsilon^{2}}\right)$.

The link field Lagrangian in unitary gauge reads

$$
\mathcal{L}_{\Omega}=\frac{1}{4} f_{\Omega}^{2}\left(\tilde{a}_{\mu}^{B}-\tilde{A}_{\mu}^{B}\right)^{2}+\frac{1}{4} f_{\Omega_{X}}^{2}\left(\tilde{x}_{\mu}-\tilde{X}_{\mu}\right)^{2},
$$

where we allowed for all possible external source fields on site-0. Turning on only those that are dynamical as in eq. (2.7), we have

$$
\begin{aligned}
\mathcal{L}_{\Omega}= & \frac{1}{2} m_{\rho}^{2} \sum_{i=1}^{3}\left(t_{\theta} w_{L \mu}^{i}-A_{L \mu}^{i}\right)^{2}+\frac{1}{2} m_{\rho}^{2}\left(t_{\theta} w_{R \mu}^{3}-A_{R \mu}^{3}\right)^{2}+\frac{1}{2} m_{X}^{2}\left[\left(g_{x} / g_{X}\right) x_{\mu}-X_{\mu}\right]^{2} \\
& +\frac{1}{2} m_{\rho}^{2} \sum_{k=1}^{2} A_{R \mu}^{k} A_{R}^{k \mu}+\frac{1}{2} m_{\rho}^{2} \sum_{a=1}^{4} A_{\mu}^{\hat{a}} A^{\hat{a} \mu}
\end{aligned}
$$

where we denoted by $A_{L \mu}^{i}$ and $A_{R \mu}^{i}$ the composite spin- 1 fields associated with the $\mathrm{SU}(2)_{L}$ and $\mathrm{SU}(2)_{R}$ factors in $\mathrm{SO}(5)$, respectively, defined

$$
t_{\theta}=\frac{g_{0}}{g_{\rho}}
$$

and

$$
m_{\rho}^{2}=\frac{1}{2} g_{\rho}^{2} f_{\Omega}^{2}, \quad m_{X}^{2}=\frac{1}{2} g_{X}^{2} f_{\Omega_{X}}^{2}
$$

and rescaled the fields according to $\tilde{w}_{L, R}=g_{0} w_{L, R}, \tilde{A}_{L, R}=g_{\rho} A_{L, R}, \tilde{x}=g_{x} x$ and $\tilde{X}=g_{X} X$ for canonical normalization. Recall that $\tilde{w}_{R \mu}^{3}$ and $\tilde{x}_{\mu}$ are written in terms of $\tilde{b}_{\mu}$ as given after eq. (2.7). By going to the mass eigenbasis, we can then identify (in the limit that $\langle h\rangle=0)$, the following massless fields:

$$
W_{L \mu}^{i}=c_{\theta} w_{L \mu}^{i}+s_{\theta} A_{L \mu}^{i}, \quad \text { for } \quad i=1,2,3,
$$

and

$$
B_{\mu}=\frac{1}{\sqrt{1+t_{\theta_{\rho}^{\prime}}^{2}+t_{\theta_{X}^{\prime}}^{2}}}\left[b_{\mu}+t_{\theta_{\rho}^{\prime}} A_{R \mu}^{3}+t_{\theta_{X}^{\prime}} X_{\mu}\right],
$$

where $t_{\theta_{\rho}^{\prime}}=g_{0}^{\prime} / g_{\rho}$ and $t_{\theta_{X}^{\prime}}=g_{0}^{\prime} / g_{X}$. These are then identified with the SM gauge fields, and acquire masses when $\langle h\rangle=v$. Indeed, one finds that

$$
m_{Z} \approx \frac{1}{2} \sqrt{g^{2}+g^{\prime 2}} \epsilon f_{h}, \quad \text { hence } \quad v_{S M}=246 \mathrm{GeV} \simeq \epsilon f_{h} .
$$

One can also identify the SM gauge couplings:

$$
g=c_{\theta} g_{0}=\left(\frac{1}{g_{0}^{2}}+\frac{1}{g_{\rho}^{2}}\right)^{-1 / 2}, \quad g^{\prime}=\frac{g_{0}^{\prime}}{\sqrt{1+t_{\theta_{\rho}^{\prime}}^{2}+t_{\theta_{X}^{\prime}}^{2}}}=\left(\frac{1}{g_{0}^{\prime 2}}+\frac{1}{g_{\rho}^{2}}+\frac{1}{g_{X}^{2}}\right)^{-1 / 2} .
$$

We note here, for later use, that in the case that $g_{X}=g_{0}^{\prime} g_{\rho} / \sqrt{g_{0}^{2}-g_{0}^{\prime 2}}$ one has that $t_{\theta}=g_{0} / g_{\rho}=g_{x} / g_{X}$, i.e. the ratios of elementary to composite couplings in the two sites 
coincide for the $\mathrm{SO}(5)$ and $\mathrm{U}(1)_{X}$ factors. In this case the usual Weinberg angle coincides with the naive elementary Weinberg angle: $t_{W}=g^{\prime} / g=g_{0}^{\prime} / g_{0}$.

The combinations orthogonal to eqs. (2.14) and (2.15) are massive even in the absence of the Higgs vev. For the $\mathrm{SU}(2)_{L} \times \mathrm{SO}(5)$ factor one finds states $\tilde{\rho}_{L \mu}^{i}=c_{\theta} A_{L \mu}^{i}-s_{\theta} w_{L \mu}^{i}$ ( $\mathrm{i}=1,2,3)$ with mass $m_{\tilde{\rho}}^{2}=\left(1+t_{\theta}^{2}\right) m_{\rho}^{2}$; the other fields in $\mathrm{SO}(5)$, that do not mix with elementary fields, correspond to two (charged) fields in $\mathrm{SU}(2)_{R} \subset \mathrm{SO}(5)$ with mass $m_{\rho}$, and four fields associated with the broken $\mathrm{SO}(5) / \mathrm{SO}(4)$ generators, with squared masses

$$
m_{a}^{2}=\frac{1}{2} g_{\rho}^{2}\left(f_{\Omega}^{2}+f_{1}^{2}\right)
$$

the latter term arising from $\mathcal{L}_{\mathrm{NGB}}$ in eq. (2.3). There are also two massive neutral resonances arising from the "hypercharge" gauge sector. Assuming that $m_{X}=m_{\rho}$, the expressions for the latter simplify considerably and one finds that the state $\propto t_{\theta_{X}^{\prime}} A_{R \mu}^{3}-t_{\theta_{\rho}^{\prime}} X_{\mu}$ has mass $m_{\rho}$ while the state $\propto t_{\theta_{\rho}^{\prime}} A_{R \mu}^{3}+t_{\theta_{X}^{\prime}} X_{\mu}-\left(t_{\theta_{\rho}^{\prime}}^{2}+t_{\theta_{X}^{\prime}}^{2}\right) b_{\mu}$ has mass squared $\left[1+t_{\theta_{\rho}^{\prime}}^{2}+t_{\theta_{X}^{\prime}}^{2}\right] m_{\rho}^{2}$. All of the above states receive small corrections when $\langle h\rangle$ is turned on. For completeness, we give the full mass matrices in appendix B.

\subsection{Fermionic sector}

On site-0 we consider a set of massless chiral fields $\psi$ with the same quantum numbers as the fermions of the SM. As explained earlier, often these will be extended to full $G_{0}^{\prime}$ multiplets by the introduction of additional fermionic sources. On site- 1 we include a set of massive Dirac fermions $\Psi^{(r)}$ arising from the strong dynamics, transforming in different representations $r$ of $G_{1}$. The fermions on site- 0 and site- 1 can be connected by the $\sigma$-model fields $\Omega$ and $\Omega_{X}$. Similarly, fermions in different representations on site- 1 can be connected by the NGB fields in $U$. The generic form of the fermion Lagrangian at quadratic order in the fermion fields that we consider in this work is

$$
\begin{aligned}
\mathcal{L}_{f}= & i \bar{\psi} \not D_{0} \psi+\bar{\Psi}^{(r)}\left(i \not D_{1}-m_{r}\right) \Psi^{(r)}+m^{(r s)} \bar{\Psi}_{L}^{(r)} U P^{(r s)} U^{\dagger} \Psi_{R}^{(s)} \\
& +\Delta^{(r)} \bar{\psi}^{(r)} \Omega\left[\Omega_{X}\right]^{q_{r}} \Psi^{(r)}+\text { h.c. }
\end{aligned}
$$

Here $D_{\mu 0}$ and $D_{\mu 1}$ are the covariant derivatives on sites-0 and 1 (i.e. carrying the corresponding elementary or composite gauge fields) and $P^{(r s)}$ is a projector in the space of representations of $\mathcal{H}_{1}$. Note that besides the "diagonal" fermion masses, $m_{r}$, the NGB's can allow additional "non-diagonal" mass terms coupling different fermion representations. From the point of view of the fermion field content, these bear some similarity with the Yukawa terms of the SM. By some simple algebraic manipulations, this term can be written in terms of the field $\Phi$ plus mixing terms between composite fermions in the same representation of $\mathrm{SO}(5)$. In the next section we will show them explicitly for each fermion embedding. The last term in eq. (2.19) leads to mixing between the elementary and composite fields, and realizes the idea of partial compositeness in the fermion sector. This term is only written for pairs of elementary and composite fermions with the same quantum numbers under $G_{0}^{\prime}$ and $G_{1}$ [here $q_{r}$ denotes the common charge of $\psi^{(r)}$ under $\mathrm{U}(1)_{x}$ and $\Psi^{(r)}$ under $\left.\mathrm{U}(1)_{X}\right]$. Note that this last term violates the $G_{0}^{\prime} \times G_{1}$ symmetry explicitly only after the non-dynamical source fields in $\psi^{(r)}$ are set to zero. The precise form of the 
above Lagrangian depends on the representations of the fermionic resonances which would be determined by the strongly coupled UV completion. In the absence of such an explicit theory, we will study several possibilities based on the lowest dimensional representations of $\mathrm{SO}(5)$. We will provide the detailed forms of the Lagrangians in section 3.

A comment regarding the structure of the third term that contains the interactions between fermions and the NGB's parametrized by $U$ is in order. As will be discussed in section 5 and explicitly shown in section 3, we will not consider the most general mass terms. Rather, in order to obtain a finite Higgs potential $V_{H}$ we have imposed some constraints. By $\Psi_{L}$ we mean the Left-handed component of the fields $\Psi$ on site- 1 that mix with the fields $\psi_{L}$ on site-0, whereas $\Psi_{R}^{\prime}$ is the Right-handed component of the fields $\Psi$ on site-1 that mix with $\psi_{R}$ on site-0. Therefore $m^{(r s)}$ will only connect $\Psi_{L}^{(r)}$ and $\Psi_{R}^{(s)}$, but there are neither terms of type $\bar{\Psi}_{R}^{(r)} U P^{(r s)} U^{\dagger} \Psi_{L}^{(s)}$ nor of type $\bar{\Psi}_{L}^{(r)} U P^{(r s)} U^{\dagger} \Psi_{R}^{(s)}$.

Also, to avoid large corrections to $Z b_{L} \bar{b}_{L}$ we will embed $Q$, the composite multiplet mixing with $q_{L}$, in a multiplet such that: $T_{L}=T_{R}$ and $T_{L}^{3}=T_{R}^{3}=-1 / 2$ for $Q_{d}$, with $T_{L, R}$ the $\mathrm{SU}(2)_{L, R}$ generators and $Q_{d}$ the component mixing with $b_{L}$. This means that $Q$ contains a $(\mathbf{2}, \mathbf{2})$ of $\mathrm{SU}(2)_{L} \times \mathrm{SU}(2)_{R}$. The smallest irreducible representations of $\mathrm{SO}(5)$ satisfying this condition are the fundamental 5, the adjoint (antisymmetric) $\mathbf{1 0}$ and the (symmetric) 14. The $\mathrm{U}(1)_{X}$ charge is fixed by demanding that the correct hypercharge be reproduced, where $Y=T_{R}^{3}+X$, leading to $X=2 / 3$. For the composite multiplet $U$ $(D)$ mixing with $u_{R}\left(d_{R}\right)$ we will consider several possibilities, but we will choose those that allow to write a Yukawa term $\bar{Q} \Phi^{n} U\left(\bar{Q} \Phi^{n} D\right)$ that is a singlet of $G_{1}$ and contain a $\mathbf{1}_{2 / 3}\left(\mathbf{1}_{-1 / 3}\right)$ of $\mathrm{SU}(2)_{L} \times \mathrm{U}(1)_{Y}$. We will consider the following models: $\mathrm{MCHM}_{5}$ (all the fermions in 5), $\mathrm{MCHM}_{10}$ (all the fermions in 10), and models involving more than one representation: $\mathrm{MCHM}_{10-5-10}, \mathrm{MCHM}_{5-5-10}, \mathrm{MCHM}_{5-10-10}, \mathrm{MCHM}_{14-14-10}$ and $\mathrm{MCHM}_{14-1-10}$, with notation $\mathrm{MCHM}_{Q-U-D}$ (see also refs. [30-34]).

Since the BR of the Higgs decaying to $\tau^{+} \tau^{-}$is not negligible, we will also consider the leptonic sector. For each generation we include two multiplets of composite fermions: $L$ and $E$, mixing with the elementary leptons $\ell_{L}$ and $e_{R}$ respectively. These composite leptons are singlets of $\mathrm{SU}(3)_{C}$ and, for each model, we choose their $\mathrm{SO}(5)$ embedding copying that of $Q$ and $D$, again with $X$ chosen to obtain $Y=T_{R}^{3}+X$.

\subsection{The low-energy effective theory}

In order to make contact with measurements at current energies, it is useful to integrate out the heavy resonances in the previous model. We will present in this section the result of integrating out the spin- 1 resonances, which is common to the various models we consider and illustrates the general procedure. In section 3 we present the result of integrating out the heavy fermionic sector in the different models of interest.

In order to simplify the computations it is useful to start with all elementary fields as non-dynamical and filling complete $G_{0}^{\prime}=\mathrm{SO}(5) \times \mathrm{U}(1)_{x}$ representations, as discussed in subsection 2.1 above. Since in this limit the full theory has an exact global $\mathrm{SO}(5) \times \mathrm{U}(1)_{X}$ symmetry, corresponding to the diagonal group of $G_{0}^{\prime} \times G_{1}$ (due to the vev of the link fields), the effective theory for these external sources must take a fully $\mathrm{SO}(5) \times \mathrm{U}(1)_{X}$ 
form. Listing all the invariant terms that are quadratic in the external gauge fields, we must obtain (in momentum space):

$$
\mathcal{L}_{\text {eff }}^{\text {sources }}=\frac{1}{2} \Pi_{A}^{(0)} \operatorname{tr}\left(\tilde{a}_{\mu} \tilde{a}^{\mu}\right)+\frac{1}{2} \Pi_{A}^{(2)} \Phi^{T} \tilde{a}_{\mu} \tilde{a}^{\mu} \Phi+\frac{1}{2} \Pi_{X}^{(0)} \tilde{x}_{\mu} \tilde{x}^{\mu}
$$

for some functions $\Pi_{A}^{(0)}\left(p^{2}\right), \Pi_{A}^{(2)}\left(p^{2}\right)$ and $\Pi_{X}^{(0)}\left(p^{2}\right)$. In the limit that $\langle h\rangle=0$, i.e. $\Phi=$ $\{0,0,0,0,1\}^{T}$, this becomes

$$
\left.\mathcal{L}_{\text {eff }}^{\text {sources }}\right|_{h=0}=\frac{1}{2} \Pi_{A}^{(0)} \tilde{a}_{\mu}^{j} \tilde{a}^{j \mu}+\frac{1}{2}\left(\Pi_{A}^{(0)}+\frac{1}{2} \Pi_{A}^{(2)}\right) \tilde{a}_{\mu}^{\hat{b}} \tilde{a}^{\hat{b} \mu}+\frac{1}{2} \Pi_{X}^{(0)} \tilde{x}_{\mu} \tilde{x}^{\mu}
$$

where $j=1, \ldots 6$ and $b=1,2,3,4$ label the two $\mathrm{SO}(4)$ representations in the adjoint of $\mathrm{SO}(5): \mathbf{1 0}=\mathbf{6}+\mathbf{4}$. We can then integrate out the heavy spin-1 resonances from $\mathcal{L}=\mathcal{L}_{\text {gauge }}+\mathcal{L}_{\Omega}+\mathcal{L}_{\mathrm{NGB}}$ [eqs. $\left.(2.3)-(2.7)\right]$ in the limit $\langle h\rangle=0$ and in unitary gauge, and identify $\Pi_{A}^{(0)}, \Pi_{A}^{(2)}$ and $\Pi_{X}^{(0)}$. The equations of motion for the heavy fields simply read

$$
\tilde{A}_{\mu}^{j}=-\frac{m_{\rho}^{2}}{p^{2}-m_{\rho}^{2}} \tilde{a}_{\mu}^{j}, \quad \tilde{A}_{\mu}^{\hat{b}}=-\frac{m_{\rho}^{2}}{p^{2}-m_{a}^{2}} \tilde{a}_{\mu}^{\hat{b}}, \quad \tilde{X}_{\mu}=-\frac{m_{X}^{2}}{p^{2}-m_{X}^{2}} \tilde{x}_{\mu},
$$

where $m_{\rho}$ and $m_{X}$ were defined in eq. (2.13), and $m_{a}$ was defined in eq. (2.18). Replacing back in the original Lagrangian, we find

$$
\Pi_{A}^{(0)}=\hat{\Pi}_{6}, \quad \Pi_{A}^{(2)}=2\left(\hat{\Pi}_{4}-\hat{\Pi}_{6}\right), \quad \Pi_{X}^{(0)}=\hat{\Pi}_{X},
$$

where

$$
\hat{\Pi}_{6}=\frac{p^{2} m_{\rho}^{2}}{g_{\rho}^{2}\left(p^{2}-m_{\rho}^{2}\right)}, \quad \hat{\Pi}_{4}=\frac{m_{\rho}^{2}\left(p^{2}+m_{\rho}^{2}-m_{a}^{2}\right)}{g_{\rho}^{2}\left(p^{2}-m_{a}^{2}\right)}, \quad \hat{\Pi}_{X}=\frac{p^{2} m_{X}^{2}}{g_{X}^{2}\left(p^{2}-m_{X}^{2}\right)} .
$$

Going back to eq. (2.20) evaluated for an arbitrary Higgs configuration, and keeping only the sources corresponding to the SM gauge fields, as described after eq. (2.7), one finds in an obvious notation:

$$
\mathcal{L}_{\text {eff }}=\frac{1}{2} \sum_{i=1}^{3} \Pi_{\tilde{w}_{L}^{i}} \tilde{w}_{L \mu}^{i} \tilde{w}_{L}^{i \mu}+\Pi_{\tilde{w}_{L}^{3} \tilde{b}} \tilde{w}_{L \mu}^{3} b^{\mu}+\frac{1}{2} \Pi_{\tilde{b}} \tilde{b}_{\mu} \tilde{b}^{\mu}
$$

where

$$
\begin{aligned}
\Pi_{\tilde{w}_{L}^{i}} & =\Pi_{A}^{(0)}+\frac{1}{4} \Pi_{A}^{(2)} \sin ^{2}\left(h / f_{h}\right), \quad \Pi_{\tilde{w}_{L}^{3} \tilde{b}}=-\frac{1}{4} \Pi_{A}^{(2)} \sin ^{2}\left(h / f_{h}\right), \\
\Pi_{\tilde{b}} & =\Pi_{X}^{(0)}+\Pi_{A}^{(0)}+\frac{1}{4} \Pi_{A}^{(2)} \sin ^{2}\left(h / f_{h}\right) .
\end{aligned}
$$

These correlators, which are valid to all orders in momentum as well as on the Higgs vev will be useful when evaluating the Higgs potential in section 5 . 


\section{Models based on the 1, 5, 10 and 14 reps. of $\mathrm{SO}(5)$}

In this section, we present a summary of the models we consider in this work, which differ in the $\mathrm{SO}(5)$ representations of the fermionic resonances arising from the strongly interacting sector. We start with a few general comments, and then describe each model in turn. The reader may want to read only the first part of this section and skip to section 4, coming back to subsections 3.1-3.7 only if further details are desired.

In unitary gauge the fermion Lagrangian can be written as:

$$
\begin{aligned}
\mathcal{L}_{f}= & \sum_{\psi=q_{L}, u_{R}, d_{R}} Z_{\psi} \bar{\psi} i \not D \psi+\bar{q}_{L} \Delta_{q} Q_{R}+\bar{u}_{R} \Delta_{u} U_{L}+\bar{d}_{R} \Delta_{d} D_{L}+\text { h.c. } \\
& +\sum_{\Psi=Q, U, D} \bar{\Psi}\left(i \not D-m_{\Psi}\right) \Psi+m_{y_{u}} \bar{Q}_{L} U_{R}+m_{y_{d}} \bar{Q}_{L} D_{R}+\mathcal{L}_{y}\left(Q_{L}, U_{R}, D_{R}, \Phi\right)+\text { h.c. }
\end{aligned}
$$

Depending on the fermion embedding, the terms $m_{y_{u}} \bar{Q}_{L} U_{R}+m_{y_{d}} \bar{Q}_{L} D_{R}$ can contain a gauge singlet or not. They are present only in the former case. The explicit form of the Yukawa terms also depends on the fermion embedding, and will be specified for each model below. ${ }^{8}$ For the $\mathrm{MCHM}_{5}$ it is necessary to include two different composite fermions $Q^{u}$ and $Q^{d}$ that mix with the elementary doublet $q_{L}$. In this case, we replace $\bar{q}_{L} \Delta_{q} Q_{R} \rightarrow \bar{q}_{L} \Delta_{q^{u}} Q_{R}^{u}+$ $\bar{q}_{L} \Delta_{q^{d}} Q_{R}^{d}$ and $m_{y_{u}} \bar{Q}_{L} U_{R}+m_{y_{d}} \bar{Q}_{L} D_{R} \rightarrow m_{y_{u}} \bar{Q}_{L}^{u} U_{R}+m_{y_{d}} \bar{Q}_{L}^{d} D_{R}$ above. However, for the other models a single $Q$ is sufficient, as written in eq. (3.1).

Integrating out the composite resonances we obtain an effective theory involving the elementary degrees of freedom only, in complete analogy to the procedure presented in section 2.4 for the spin- 1 case. The fermions are in complete irreducible representations $r_{5}$ of $\mathrm{SO}(5)$. However, due to the spontaneous breaking $\mathrm{SO}(5) \rightarrow \mathrm{SO}(4)$ in the composite sector, each fermion is in general split into several irreducible representations $r_{4}$ of $\mathrm{SO}(4)$ : $\psi^{r_{5}}=\sum_{r_{4}} \alpha_{r_{5}, r_{4}} \psi^{r_{4}}$, with $\alpha_{r_{5}, r_{4}}$ the coefficients associated to the decomposition. Thus, before EWSB, and taking $\langle\Phi\rangle=\Phi_{0}($ i.e. $h=0)$, one can write the effective Lagrangian as:

$$
\left.\mathcal{L}_{\text {eff }}\right|_{h=0}=\sum_{\psi=q_{L}, u_{R}, d_{R}} \sum_{r_{4}} \bar{\psi}^{\left(r_{4}\right)} \not p\left(Z_{\psi}+\hat{\Pi}_{\psi}^{\left(r_{4}\right)}\right) \psi^{\left(r_{4}\right)}+\sum_{\psi=u, d} \sum_{r_{4}} \bar{q}_{L}^{\left(r_{4}\right)} \hat{M}_{\psi^{\left(r_{4}\right)}} \psi_{R}^{\left(r_{4}\right)}+\text { h.c. }
$$

The explicit form of the correlators $\hat{\Pi}_{\psi}^{r_{4}}$ and $\hat{M}_{\psi}^{r_{4}}$ are given in the appendix for the different models.

It is then simple to compare to the correlators of an effective Lagrangian, $\mathcal{L}_{\text {eff }}$, written in fully $\mathrm{SO}(5)$ invariant form with the help of an arbitrary $\Phi$ (one should list all possible $\mathrm{SO}(5) \times \mathrm{U}(1)_{X}$ invariant operators that are quadratic in the external fermionic sources, which depends on the specific model in question). If one then retains the SM degrees of freedom only, the effective Lagrangian for the elementary fermions takes the form

$$
\begin{aligned}
\mathcal{L}_{\text {eff }}= & \bar{u}_{L} \not p\left(Z_{q}+\Pi_{u_{L}}\right) u_{L}+\bar{d}_{L} \not p\left(Z_{q}+\Pi_{d_{L}}\right) d_{L}+\bar{u}_{R} \not p\left(Z_{u}+\Pi_{u_{R}}\right) u_{R} \\
& +\bar{d}_{R} \not p\left(Z_{d}+\Pi_{d_{R}}\right) d_{R}+\bar{u}_{L} M_{u} u_{R}+\bar{d}_{L} M_{d} d_{R}+\text { h.c. }
\end{aligned}
$$

\footnotetext{
${ }^{8}$ These Yukawa interactions are not yet the SM Yukawa interactions, but will give rise to them. Therefore, we will refer to them as "proto-Yukawa" interactions.
} 
The correlators $\Pi_{\psi}$ and $M_{\psi}$ can be expressed in terms of the correlators of the $\mathrm{SO}(4)$ symmetric theory $\hat{\Pi}_{\psi}^{r_{4}}$ and $\hat{M}_{\psi}^{r_{4}}$, and have an explicit (and generally simple) dependence on $s_{h}=\sin h / f_{h}$ and $c_{h}=\cos h / f_{h}$. We show below the full expressions for each specific model.

The spectrum of fermions that mix with the SM ones (as well as the masses of the SM degrees of freedom) is given by the zeroes of the quadratic operator

$$
\text { Zero }\left\{p^{2}\left[Z_{q}+\Pi_{\psi_{L}}\left(p^{2}\right)\right]\left[Z_{\psi}+\Pi_{\psi_{R}}\left(p^{2}\right)\right]-\left|M_{\psi}\left(p^{2}\right)\right|^{2}\right\}, \quad \psi=u, d .
$$

The SM states, being lighter than the compositeness scale, can be obtained by expanding eq. (3.4) to $\mathcal{O}\left(p^{2}\right)$, leading to

$$
m_{\psi}^{(0)} \simeq\left|M_{\psi}(0)\right|\left\{\left[Z_{q}+\Pi_{\psi_{L}}(0)\right]\left[Z_{\psi}+\Pi_{\psi_{R}}(0)\right]-\left.2\left|M_{\psi}(0)\right| \frac{d\left|M_{\psi}\left(p^{2}\right)\right|}{d p^{2}}\right|_{p^{2}=0}\right\}^{-1 / 2}, \quad \psi=u, d,
$$

We have used the superindex (0) for the lightest states, since in the absence of mixings they are massless.

Similarly, the Yukawa coupling of these states to (a single) Higgs boson can be obtained by differentiating with respect to $v$ :

$$
y_{\psi}^{(0)} \simeq \frac{d m_{\psi}^{(0)}}{d v}, \quad \psi=u, d
$$

This coupling depends on the model, but since the vev dependence of the correlators is simple (it is encoded in $s_{h}$ and $c_{h}$ in the formulas given in the following subsections), we can derive simple expression in terms of the correlators, that will be given for each model below.

A very important combination for the phenomenology is the function $y_{\psi}^{(0)} / m_{\psi}^{(0)}$. To leading order in $\epsilon$ it can be approximated by:

$$
\frac{y_{\psi}^{(0)}}{m_{\psi}^{(0)}} \simeq \frac{F_{\psi}(\epsilon)}{\epsilon f_{h}}\left[1+\mathcal{O}\left(\epsilon^{2}\right)\right], \quad \psi=u, d
$$

where the $F_{\psi}(\epsilon)$ depends only on $\epsilon$ (as well as on the fermion representation) and will be given in section $4 .^{9}$ The $\mathcal{O}\left(\epsilon^{2}\right)$ correction (which also depends on other microscopic parameters) determines the deviation compared with the simple and compact leading approximation. The above relation is intimately connected to certain sum rules that have been already observed in the literature $[19,35,36]$. We will comment further on this in section 6 .

As will be shown below, different models lead to different sizes for the $\mathcal{O}\left(\epsilon^{2}\right)$ term. Multiplying eq. (3.7) by $v_{S M}$, and using eq. (2.16), we can obtain the ratio between the Yukawa couplings in the MCHM and in the SM:

$$
\frac{y_{\psi}^{(0)}}{y_{\psi}^{S M}} \simeq F_{\psi}(\epsilon)\left[1+\mathcal{O}\left(\epsilon^{2}\right)\right], \quad \psi=u, d,
$$

\footnotetext{
${ }^{9}$ The are exceptions to this statement, with additional dependence on the Yukawa couplings on the r.h.s. of eq. (3.7). We consider one such detailed example in this work and mention a few others. However, in certain limits the above discussion often applies.
} 
showing that deviations from $F_{\psi}(\epsilon)$ are suppressed by $\mathcal{O}\left(\epsilon^{2}\right)$. This correction depends also on the fermionic mixings in the following way: $\mathcal{O}\left(\epsilon^{2} s_{\psi_{L}}^{2}, \epsilon^{2} s_{\psi_{R}}^{2}\right)$, requiring in general the mixing of both chiralities to be small to ensure extra suppression factors. However, for some models the structure inherited from the fermion embedding is such that the correction involves just one chirality to leading order: $\mathcal{O}\left(\epsilon^{2} s_{\psi_{L}}^{2}\right)$ or $\mathcal{O}\left(\epsilon^{2} s_{\psi_{R}}^{2}\right)$. In those cases an extra suppression can be achieved with small mixing for one chirality only. Note also that the above corrections do not take the form claimed in [19], i.e. $\mathcal{O}\left(\epsilon^{2} m_{\psi}^{2}\right) \sim \mathcal{O}\left(\epsilon^{2} s_{\psi_{L}}^{2} s_{\psi_{R}}^{2}\right)$, where $m_{\psi}$ denotes the mass of the SM field (this has also been observed in ref. [21]). Thus, the bottom quark, in particular, can give corrections that are larger than expected, as will be illustrated in section 6 .

\section{$3.1 \quad \mathrm{MCHM}_{5}$}

In this model we consider 4 composite fermions for each generation: $Q^{u}, U \sim \mathbf{5}_{2 / 3}$ and $Q^{d}, D \sim \mathbf{5}_{-1 / 3}$, where the subindex denotes the $\mathrm{U}(1)_{X}$ charge. In unitary gauge the Yukawa terms of the fermion Lagrangian (3.1) read:

$$
\mathcal{L}_{y}=y_{u}\left(\bar{Q}_{L}^{u} \Phi\right)\left(\Phi^{\dagger} U_{R}\right)+y_{d}\left(\bar{Q}_{L}^{d} \Phi\right)\left(\Phi^{\dagger} D_{R}\right) .
$$

In this case $q_{L}$ mixes with two composite fermions: $Q^{u}$ and $Q^{d}$. The bottom mass can result from small $\Delta_{q^{d}}$ and/or small $\Delta_{d}$.

The correlators of the effective Lagrangian (3.3) are:

$$
\begin{aligned}
\Pi_{u_{L}} & =\Pi_{q^{u}}^{0}+\Pi_{q^{d}}^{0}+\Pi_{q^{u}}^{1} \frac{s_{h}^{2}}{2}, & \Pi_{d_{L}} & =\Pi_{q^{u}}^{0}+\Pi_{q^{d}}^{0}+\Pi_{q^{d}}^{1} \frac{s_{h}^{2}}{2}, \\
\Pi_{u_{R}} & =\Pi_{u}^{0}+\Pi_{u}^{1} c_{h}^{2}, & \Pi_{d_{R}} & =\Pi_{d}^{0}+\Pi_{d}^{1} c_{h}^{2}, \\
M_{u} & =m_{u}^{1} \frac{s_{h} c_{h}}{\sqrt{2}}, & M_{d} & =m_{d}^{1} \frac{s_{h} c_{h}}{\sqrt{2}} .
\end{aligned}
$$

where the $\Pi_{\psi}^{i}$ are defined by

$$
\begin{aligned}
\mathcal{L}_{\text {eff }}^{\text {sources }}= & \bar{q}_{L}^{u} \not p \Pi_{q^{u}}^{0} q_{L}^{u}+\bar{q}_{L}^{d} \not \Pi_{q^{d}}^{0} q_{L}^{d}+\bar{u}_{R} \not p \Pi_{u}^{0} u_{R}+\bar{d}_{R} \not \Pi_{d}^{0} d_{R}+\left(\bar{q}_{L}^{u} \Phi\right) \not p \Pi_{q^{u}}^{1}\left(\Phi^{\dagger} q_{L}^{u}\right) \\
& +\left(\bar{q}_{L}^{d} \Phi\right) \not p \Pi_{q^{d}}^{1}\left(\Phi^{\dagger} q_{L}^{d}\right)+\left(\bar{u}_{R} \Phi\right) \not p \Pi_{u}^{1}\left(\Phi^{\dagger} u_{R}\right)+\left(\bar{d}_{R} \Phi\right) \not p \Pi_{d}^{1}\left(\Phi^{\dagger} d_{R}\right) \\
& +m_{u}^{0} \bar{q}_{L}^{u} u_{R}+m_{d}^{0} \bar{q}_{L}^{d} d_{R}+m_{u}^{1}\left(\bar{q}_{L}^{u} \Phi\right)\left(\Phi^{\dagger} u_{R}\right)+m_{d}^{1}\left(\bar{q}_{L}^{d} \Phi\right)\left(\Phi^{\dagger} d_{R}\right)+\text { h.c. }
\end{aligned}
$$

The superindex "sources" serves as a reminder that here the $q_{L}^{u}, q_{L}^{d}, u_{R}$ and $d_{R}$ fill complete $\mathrm{SO}(5)$ multiplets and that all components are to be treated as external sources. One must still add "bare" kinetic terms for the dynamical fields on site-0, i.e. those with SM quantum numbers, as in eq. (3.3). Since a $\mathbf{5}$ of $\mathrm{SO}(5)$ decomposes under $\mathrm{SO}(4)$ as $\mathbf{5} \sim \mathbf{1}+\mathbf{4}$, one finds

$$
\begin{aligned}
& \Pi_{q^{u}}^{0}=\hat{\Pi}_{q^{u(4)}}, \\
& \Pi_{q^{d}}^{0}=\hat{\Pi}_{q^{d(4)}}, \\
& \Pi_{d}^{0}=\hat{\Pi}_{d^{(4)}}, \\
& \Pi_{q^{u}}^{1}=\hat{\Pi}_{q^{u(1)}}-\hat{\Pi}_{q^{u(4)}}, \\
& \Pi_{q^{d}}^{1}=\hat{\Pi}_{q^{d(1)}}-\hat{\Pi}_{q^{d(4)}}, \\
& \Pi_{d}^{1}=\hat{\Pi}_{d^{(1)}}-\hat{\Pi}_{d^{(4)}}, \\
& \Pi_{u}^{0}=\hat{\Pi}_{u^{(4)}}, \\
& m_{u}^{0}=\hat{M}_{u(4)}, \\
& m_{d}^{0}=\hat{M}_{d^{(4)}}, \\
& \Pi_{u}^{1}=\hat{\Pi}_{u^{(1)}}-\hat{\Pi}_{u^{(4)}}, \\
& m_{u}^{1}=\hat{M}_{u^{(1)}}-\hat{M}_{u^{(4)}}, \\
& m_{d}^{1}=\hat{M}_{d^{(1)}}-\hat{M}_{d^{(4)}} \text {. }
\end{aligned}
$$

where the hatted correlators are given in appendix C.1. 
Using these correlators we can compute the prediction for $y_{\psi}^{(0)} / m_{\psi}^{(0)}$ :

$$
\begin{aligned}
\frac{y_{t}^{(0)}}{m_{t}^{(0)}}-\frac{F_{t}}{s_{h} f_{h}} \simeq \frac{s_{h}}{f_{h}} \frac{2\left|m_{u}^{1}(0)\right|\left|m_{u}^{1}(0)\right|^{\prime}-\left[Z_{u}+\Pi_{u}^{0}(0)+\Pi_{u}^{1}(0)\right] \Pi_{q^{u}}^{1}+2\left[Z_{q}+\Pi_{q}^{0}(0)\right] \Pi_{u}^{1}}{2\left[Z_{u}+\Pi_{u}^{0}(0)+\Pi_{u}^{1}(0)\right]\left[Z_{q}+\Pi_{q}^{0}(0)\right]} \\
\frac{y_{b}^{(0)}}{m_{b}^{(0)}}-\frac{F_{b}}{s_{h} f_{h}} \simeq \frac{s_{h}}{f_{h}} \frac{2\left|m_{d}^{1}(0)\right|\left|m_{d}^{1}(0)\right|^{\prime}-\left[Z_{d}+\Pi_{d}^{0}(0)+\Pi_{d}^{1}(0)\right] \Pi_{q^{d}}^{1}+2\left[Z_{q}+\Pi_{q}^{0}(0)\right] \Pi_{d}^{1}}{2\left[Z_{d}+\Pi_{d}^{0}(0)+\Pi_{d}^{1}(0)\right]\left[Z_{q}+\Pi_{q}^{0}(0)\right]}
\end{aligned}
$$

where $\left|m_{\psi}^{i}(0)\right|^{\prime} \equiv\left|d m_{\psi}^{i}\left(p^{2}\right)\right| /\left.d p^{2}\right|_{p^{2}=0}$. Taking into account that $\Pi_{\psi}^{j} \sim \Delta_{\psi}^{2}$ and $M_{\psi} \sim$ $\Delta_{q} \Delta_{\psi}$, eq. (3.14) shows that the $\mathcal{O}\left(s_{h}^{2}\right)$ correction to $y_{b}$ in this model is small. By expressing $\Delta_{\psi}$ in terms of the elementary-composite mixing angles, one sees that the correction is suppressed by $s_{q^{d}}^{2}$ or $s_{d}^{2}$. By choosing both of them small, we expect $y_{b}^{(0)} / m_{b}^{(0)}$ to be well approximated by $F_{b} / s_{h} f_{h}$ in this model. On the other hand, eq. (3.13) shows that the corrections to $y_{t}$ do not have any extra suppression factor in general, since the top mass requires both, $s_{q^{u}}$ and $s_{u} \sim \mathcal{O}(1)$. This property has important consequences for the phenomenology: one can expect corrections to loop-induced processes that depend on $y_{t}$ [gluon fusion, $h \rightarrow \gamma \gamma$ to be discussed in section 6] of $\mathcal{O}\left(s_{h}^{2}\right)$. The size of these corrections is similar for all the models. Since all of them require $s_{q}$ and $s_{u} \lesssim 1$, there can be differences of $\mathcal{O}(1)$ between them arising from the different embeddings and regions of the parameter space selected.

\section{$3.2 \quad \mathrm{MCHM}_{10}$}

From now on, we consider 3 composite fermions for each generation. In this model: $Q, U, D \sim \mathbf{1 0}_{2 / 3}$. In unitary gauge the Yukawa terms of the fermion Lagrangian (3.1) read:

$$
\mathcal{L}_{y}=y_{u} \Phi^{\dagger} \bar{Q}_{L} U_{R} \Phi+y_{d} \Phi^{\dagger} \bar{Q}_{L} D_{R} \Phi
$$

In this case $q_{L}$ mixes with a single composite fermion $Q$ and, therefore, the bottom mass requires small $\Delta_{d}$. In this model the interactions $\Phi^{\dagger} \bar{U}_{L} D_{R} \Phi$ and $\Phi^{\dagger} \bar{U}_{R} D_{L} \Phi$ are also compatible with the symmetries. However they lead to a logarithmically divergent Higgs potential, and we do not include them. Note also that we do not include terms of the form $\epsilon_{A B C D E} \Phi^{A} \bar{Q}_{L}^{B C} U_{R}^{D E}$, etc., which would break a LR symmetry, and have been studied in [37].

The correlators of the effective Lagrangian (3.3) are:

$$
\begin{array}{rlrl}
\Pi_{u_{L}} & =\Pi_{q}^{0}+\Pi_{q}^{1}\left(\frac{c_{h}^{2}}{2}+\frac{s_{h}^{2}}{4}\right), & \Pi_{d_{L}} & =\Pi_{q}^{0}+\Pi_{q}^{1} \frac{c_{h}^{2}}{2}, \\
\Pi_{u_{R}} & =\Pi_{u}^{0}+\Pi_{u}^{1} \frac{s_{h}^{2}}{4}, & \Pi_{d_{R}} & =\Pi_{d}^{0}+\Pi_{d}^{1} \frac{s_{h}^{2}}{4}, \\
M_{u} & =-m_{u}^{1} \frac{s_{h} c_{h}}{4}, & M_{d}=-m_{d}^{1} \frac{s_{h} c_{h}}{2 \sqrt{2}}
\end{array}
$$


where the $\Pi_{\psi}^{i}$ are now defined by [see also comments following eq. (3.11)]

$$
\begin{aligned}
\mathcal{L}_{\text {eff }}^{\text {sources }}= & \operatorname{Tr}\left[\bar{q}_{L} \not p \Pi_{q}^{0} q_{L}+\bar{u}_{R} \not p \Pi_{u}^{0} u_{R}+\bar{d}_{R} \not p \Pi_{d}^{0} d_{R}\right] \\
& +\Phi^{\dagger} \bar{q}_{L} \not p \Pi_{q}^{1} q_{L} \Phi+\Phi^{\dagger} \bar{u}_{R} \not p \Pi_{u}^{1} u_{R} \Phi+\Phi^{\dagger} \bar{d}_{R} \not p \Pi_{d}^{1} d_{R} \Phi \\
& +\operatorname{Tr}\left[m_{u}^{0} \bar{q}_{L} u_{R}+m_{d}^{0} \bar{q}_{L} d_{R}\right]+m_{u}^{1} \Phi^{\dagger} \bar{q}_{L} u_{R} \Phi+m_{d}^{1} \Phi^{\dagger} \bar{q}_{L} d_{R} \Phi+\text { h.c. }
\end{aligned}
$$

Since a $\mathbf{1 0}$ of $\mathrm{SO}(5)$ decomposes under $\mathrm{SO}(4)$ as $\mathbf{1 0} \sim \mathbf{4 + 6}$, we find

$$
\begin{array}{rlrl}
\Pi_{q}^{0} & =\hat{\Pi}_{q^{(6)}}, & \Pi_{u}^{0}=\hat{\Pi}_{u^{(6)}}, & \Pi_{d}^{0}=\hat{\Pi}_{d^{(6)}}, \\
\Pi_{q}^{1}=2\left(\hat{\Pi}_{q^{(4)}}-\hat{\Pi}_{q^{(6)}}\right), & \Pi_{u}^{1}=2\left(\hat{\Pi}_{u^{(4)}}-\hat{\Pi}_{u^{(6)}}\right), & \Pi_{d}^{1}=2\left(\hat{\Pi}_{d^{(4)}}-\hat{\Pi}_{d^{(6)}}\right), \\
m_{u}^{0}=\hat{M}_{u^{(6)}}, & m_{d}^{0}=\hat{M}_{d^{(6)}}, & \\
m_{u}^{1}=2\left(\hat{M}_{u^{(4)}}-\hat{M}_{u^{(6)}}\right), & m_{d}^{1}=2\left(\hat{M}_{d^{(4)}}-\hat{M}_{d^{(6)}}\right) . &
\end{array}
$$

where the hatted correlators are given in appendix C.2.

The prediction for $y_{\psi} / m_{\psi}$ is:

$$
\begin{aligned}
& \frac{y_{t}^{(0)}}{m_{t}^{(0)}}-\frac{F_{t}}{s_{h} f_{h}} \simeq \frac{s_{h}}{f_{h}} \frac{\left|m_{u}^{1}(0)\right|\left|m_{u}^{1}(0)\right|^{\prime}+\left[2 Z_{u}+2 \Pi_{u}^{0}(0)-\Pi_{u}^{1}(0)\right] \Pi_{q}^{1}-2\left[Z_{q}+\Pi_{q}^{0}(0)\right] \Pi_{u}^{1}}{4\left[Z_{u}+\Pi_{u}^{0}(0)\right]\left[2 Z_{q}+2 \Pi_{q}^{0}(0)+\Pi_{q}^{1}(0)\right]} \\
& \frac{y_{b}^{(0)}}{m_{b}^{(0)}}-\frac{F_{b}}{s_{h} f_{h}} \simeq \frac{s_{h}}{f_{h}} \frac{2\left|m_{d}^{1}(0)\right|\left|m_{d}^{1}(0)\right|^{\prime}+4\left[Z_{d}+\Pi_{d}^{0}(0)\right] \Pi_{q}^{1}-\left[2 Z_{q}+2 \Pi_{q}^{0}(0)+\Pi_{q}^{1}(0)\right] \Pi_{d}^{1}}{4\left[Z_{d}+\Pi_{d}^{0}(0)\right]\left[2 Z_{q}+2 \Pi_{q}^{0}(0)+\Pi_{q}^{1}(0)\right]} .
\end{aligned}
$$

Eq. (3.20) shows that the $\mathcal{O}\left(s_{h}^{2}\right)$ corrections to $y_{b}$ in this model can be sizable. This is because there is a term suppressed by $s_{q}^{2}$ only, but $s_{q} \sim 1$ to reproduce the top mass. Thus, we find a suppression by $s_{h}^{2}$ only.

\section{$3.3 \quad \mathrm{MCHM}_{10-5-10}$}

In this model: $Q, D \sim \mathbf{1 0}_{2 / 3}$ and $U \sim \mathbf{5}_{2 / 3}$. In unitary gauge the Yukawa terms of the fermion Lagrangian (3.1) read:

$$
\mathcal{L}_{y}=y_{u} \Phi^{\dagger} \bar{Q}_{L} U_{R}+y_{d} \Phi^{\dagger} \bar{Q}_{L} D_{R} \Phi
$$

The correlators of the effective Lagrangian (3.3) are:

$$
\begin{array}{rlrl}
\Pi_{u_{L}} & =\Pi_{q}^{0}+\Pi_{q}^{1}\left(\frac{c_{h}^{2}}{2}+\frac{s_{h}^{2}}{4}\right), & \Pi_{d_{L}} & =\Pi_{q}^{0}+\Pi_{q}^{1} \frac{c_{h}^{2}}{2}, \\
\Pi_{u_{R}} & =\Pi_{u}^{0}+\Pi_{u}^{1} c_{h}^{2}, & \Pi_{d_{R}} & =\Pi_{d}^{0}+\Pi_{d}^{1} \frac{s_{h}^{2}}{4}, \\
M_{u} & =-m_{u}^{1} \frac{s_{h}}{2}, & M_{d}=-m_{d}^{1} \frac{s_{h} c_{h}}{2 \sqrt{2}} .
\end{array}
$$

where the $\Pi_{\psi}^{i}$ are defined in analogy to eqs. (3.11) and (3.17), with the $\Phi$-dependent terms following the structure displayed in eq. (3.21) for the Yukawa terms in this model [see also comments following eq. (3.11)]. Expanding the Higgs potential in powers of $s_{h}$ and $\Delta_{\psi}$, the contribution of $M_{u}$ to the quartic coupling is of order $\mathcal{O}\left(\Delta_{\psi}^{8}\right)$ and the only contributions 
of order $\mathcal{O}\left(\Delta_{\psi}^{4}\right)$ are from $\Pi_{L}$ and $\Pi_{R}$. Therefore, in this model we expect a small selfcoupling and a very light Higgs. This fact is reflected in the tuning of the model which, after requiring the proper Higgs mass, is one order of magnitude larger than in the other models. A sizable quartic coupling demands very large mixings for the top quark, inducing departures from the analytical approximations for the Yukawa couplings. This also affects the bottom since the $b_{L}$ mixing is equal to the $t_{L}$ mixing in this model.

Using the previous decompositions of $\mathbf{5}$ and $\mathbf{1 0}$ of $\mathrm{SO}(5)$ under $\mathrm{SO}(4)$ one finds:

$$
\begin{array}{lll}
\Pi_{q}^{0}=\hat{\Pi}_{q^{(6)}}, & \Pi_{u}^{0}=\hat{\Pi}_{u^{(4)}}, & \Pi_{d}^{0}=\hat{\Pi}_{d^{(6)}}, \\
\Pi_{q}^{1}=2\left(\hat{\Pi}_{q^{(4)}}-\hat{\Pi}_{q^{(6)}}\right), & \Pi_{u}^{1}=\hat{\Pi}_{u^{(1)}}-\hat{\Pi}_{u^{(4)}}, & \Pi_{d}^{1}=2\left(\hat{\Pi}_{d^{(4)}}-\hat{\Pi}_{d^{(6)}}\right), \\
m_{u}^{0}=0, & m_{d}^{0}=\hat{M}_{d^{(6)}}, & \\
m_{u}^{1}=\sqrt{2} \hat{M}_{u^{(4)}}, & m_{d}^{1}=2\left(\hat{M}_{d^{(4)}}-\hat{M}_{d^{(6)}}\right) . &
\end{array}
$$

where the hatted correlators are given in appendix C.3.

The prediction for $y_{\psi} / m_{\psi}$ is:

$$
\begin{aligned}
\frac{y_{t}^{(0)}}{m_{t}^{(0)}}-\frac{F_{t}}{s_{h} f_{h}} \simeq \frac{s_{h}}{f_{h}} \frac{2\left|m_{u}^{1}(0)\right|\left|m_{u}^{1}(0)\right|^{\prime}+\left[Z_{u}+\Pi_{u}^{0}(0)+3 \Pi_{u}^{1}(0)\right] \Pi_{q}^{1}+4\left[Z_{q}+\Pi_{q}^{0}(0)\right] \Pi_{u}^{1}}{2\left[Z_{u}+\Pi_{u}^{0}(0)+\Pi_{u}^{1}(0)\right]\left[2 Z_{q}+2 \Pi_{q}^{0}(0)+\Pi_{q}^{1}(0)\right]}, \\
\frac{y_{b}^{(0)}}{m_{b}^{(0)}}-\frac{F_{b}}{s_{h} f_{h}} \simeq \frac{s_{h}}{f_{h}} \frac{2\left|m_{d}^{1}(0)\right|\left|m_{d}^{1}(0)\right|^{\prime}+4\left[Z_{d}+\Pi_{d}^{0}(0)\right] \Pi_{q}^{1}-\left[2 Z_{q}+2 \Pi_{q}^{0}(0)+\Pi_{q}^{1}(0)\right] \Pi_{d}^{1}}{4\left[Z_{d}+\Pi_{d}^{0}(0)\right]\left[2 Z_{q}+2 \Pi_{q}^{0}(0)+\Pi_{q}^{1}(0)\right]} .
\end{aligned}
$$

$y_{b} / m_{b}$ in this model is exactly as in the $\mathrm{MCHM}_{10}$ when expressed in terms of the correlators, although the correlators themselves are different in both models. This can be understood because the bottom mass arises from the coupling between $q$ and $d$, that share the same embedding in both models.

\section{$3.4 \mathrm{MCHM}_{5-5-10}$}

In this model: $Q, U \sim \mathbf{5}_{2 / 3}$ and $D \sim \mathbf{1 0}_{2 / 3}$. In unitary gauge the Yukawa terms of the fermion Lagrangian (3.1) read:

$$
\mathcal{L}_{y}=y_{u}\left(\bar{Q}_{L} \Phi\right)\left(\Phi^{\dagger} U_{R}\right)+y_{d} \bar{Q}_{L} D_{R} \Phi .
$$

The correlators of the effective Lagrangian (3.3) are:

$$
\begin{aligned}
\Pi_{u_{L}} & =\Pi_{q}^{0}+\Pi_{q}^{1} \frac{s_{h}^{2}}{2}, & \Pi_{d_{L}} & =\Pi_{q}^{0}, \\
\Pi_{u_{R}} & =\Pi_{u}^{0}+\Pi_{u}^{1} c_{h}^{2}, & \Pi_{d_{R}} & =\Pi_{d}^{0}+\Pi_{d}^{1} \frac{s_{h}^{2}}{4}, \\
M_{u} & =m_{u}^{1} \frac{s_{h} c_{h}}{\sqrt{2}}, & M_{d} & =m_{d}^{1} \frac{s_{h}}{2} .
\end{aligned}
$$

where the $\Pi_{\psi}^{i}$ are defined in analogy to eqs. (3.11) and (3.17), with the $\Phi$-dependent terms following the structure displayed in eq. (3.26) for the Yukawa terms in this model [see also 
comments following eq. (3.11)]. Using the previous decompositions of $\mathbf{5}$ and $\mathbf{1 0}$ of $\mathrm{SO}(5)$ under $\mathrm{SO}(4)$ :

$$
\begin{array}{lll}
\Pi_{q}^{0}=\hat{\Pi}_{q^{(4)}}, & \Pi_{u}^{0}=\hat{\Pi}_{u^{(4)}}, & \Pi_{d}^{0}=\hat{\Pi}_{d^{(6)}}, \\
\Pi_{q}^{1}=\hat{\Pi}_{q^{(1)}}-\hat{\Pi}_{q^{(4)}}, & \Pi_{u}^{1}=\hat{\Pi}_{u^{(1)}}-\hat{\Pi}_{u^{(4)}}, & \Pi_{d}^{1}=2\left(\hat{\Pi}_{d^{(4)}}-\hat{\Pi}_{d^{(6)}}\right), \\
m_{u}^{0}=\hat{M}_{u^{(4)}}, & m_{d}^{0}=0, & \\
m_{u}^{1}=\hat{M}_{u^{(1)}}-\hat{M}_{u^{(4)}}, & m_{d}^{1}=\sqrt{2} \hat{M}_{d^{(4)}} . &
\end{array}
$$

where the hatted correlators are given in appendix C.4.

The prediction for $y_{\psi} / m_{\psi}$ is:

$$
\begin{aligned}
\frac{y_{t}^{(0)}}{m_{t}^{(0)}}-\frac{F_{t}}{s_{h} f_{h}} \simeq \frac{s_{h}}{f_{h}} \frac{2\left|m_{u}^{1}(0)\right|\left|m_{u}^{1}(0)\right|^{\prime}+2\left[Z_{q}+\Pi_{q}^{0}(0)\right] \Pi_{u}^{1}-\left[Z_{u}+\Pi_{u}^{0}(0)+\Pi_{u}^{1}(0)\right] \Pi_{q}^{1}}{2\left[Z_{u}+\Pi_{u}^{0}(0)+\Pi_{u}^{1}(0)\right]\left[Z_{q}+\Pi_{q}^{0}(0)\right]} ; \\
\frac{y_{b}^{(0)}}{m_{b}^{(0)}}-\frac{F_{b}}{s_{h} f_{h}} \simeq \frac{s_{h}}{f_{h}} \frac{2\left|m_{d}^{1}(0)\right|\left|m_{d}^{1}(0)\right|^{\prime}-\left[Z_{q}+\Pi_{q}^{0}(0)\right] \Pi_{d}^{1}}{4\left[Z_{d}+\Pi_{d}^{0}(0)\right]\left[Z_{q}+\Pi_{q}^{0}(0)\right]} .
\end{aligned}
$$

For the top quark we obtain a result similar to the $\mathrm{MCHM}_{5}$. Eq. (3.30) shows that the $\mathcal{O}\left(s_{h}^{2}\right)$ corrections to $y_{b}^{(0)} / m_{b}^{(0)}$ in this model is also suppressed by $s_{d}^{2} \ll 1$.

\section{5 $\mathrm{MCHM}_{5-10-10}$}

In this model: $Q \sim \mathbf{5}_{2 / 3}$ and $U, D \sim \mathbf{1 0}_{2 / 3}$. In unitary gauge the Yukawa terms of the fermion Lagrangian (3.1) read:

$$
\mathcal{L}_{y}=y_{u} \bar{Q}_{L} U_{R} \Phi+y_{d} \bar{Q}_{L} D_{R} \Phi .
$$

In this model the interactions $\Phi^{\dagger} \bar{U}_{L} D_{R} \Phi$ and $\Phi^{\dagger} \bar{U}_{R} D_{L} \Phi$ are also compatible with the symmetries. However they lead to a logarithmically divergent Higgs potential, therefore we will not include them.

The correlators of the effective Lagrangian (3.3) are:

$$
\begin{aligned}
\Pi_{u_{L}} & =\Pi_{q}^{0}+\Pi_{q}^{1} \frac{s_{h}^{2}}{2}, & \Pi_{d_{L}} & =\Pi_{q}^{0}, \\
\Pi_{u_{R}} & =\Pi_{u}^{0}+\Pi_{u}^{1} \frac{s_{h}^{2}}{4}, & \Pi_{d_{R}} & =\Pi_{d}^{0}+\Pi_{d}^{1} \frac{s_{h}^{2}}{4}, \\
M_{u} & =-m_{u}^{1} \frac{s_{h}}{2 \sqrt{2}}, & M_{d} & =m_{d}^{1} \frac{s_{h}}{2} .
\end{aligned}
$$

where the $\Pi_{\psi}^{i}$ are defined in analogy to eqs. (3.11) and (3.17), with the $\Phi$-dependent terms following the structure displayed in eq. (3.31) for the Yukawa terms in this model [see also comments following eq. (3.11)]. Since the Higgs dependence on $M_{u}$ is the same as in the $\mathrm{MCHM}_{10-5-10}$, the behavior of the Higgs potential and the top Yukawa are similar.

Using the previous decompositions of $\mathbf{5}$ and $\mathbf{1 0}$ of $\mathrm{SO}(5)$ under $\mathrm{SO}(4)$ :

$$
\begin{array}{rlrl}
\Pi_{q}^{0} & =\hat{\Pi}_{q^{(4)}}, & \Pi_{u}^{0}=\hat{\Pi}_{u^{(6)}}, & \Pi_{d}^{0}=\hat{\Pi}_{d^{(6)}}, \\
\Pi_{q}^{1}=\hat{\Pi}_{q^{(1)}}-\hat{\Pi}_{q^{(4)}}, & \Pi_{u}^{1}=2\left(\hat{\Pi}_{u^{(4)}}-\hat{\Pi}_{u^{(6)}}\right), & \Pi_{d}^{1}=2\left(\hat{\Pi}_{d^{(4)}}-\hat{\Pi}_{d^{(6)}}\right), \\
m_{u}^{0}=0, & m_{d}^{0}=0, & \\
m_{u}^{1}=\sqrt{2} \hat{M}_{u^{(4)}}, & m_{d}^{1}=\sqrt{2} \hat{M}_{d^{(4)}} . &
\end{array}
$$


where the hatted correlators are given in appendix C.5.

The prediction for $y_{\psi} / m_{\psi}$ is:

$$
\begin{aligned}
\frac{y_{t}^{(0)}}{m_{t}^{(0)}}-\frac{F_{t}}{s_{h} f_{h}} \simeq \frac{s_{h}}{f_{h}} \frac{\left|m_{u}^{1}(0)\right|\left|m_{u}^{1}(0)\right|^{\prime}-\left[Z_{q}+\Pi_{q}^{0}(0)\right] \Pi_{u}^{1}-2\left[Z_{u}+\Pi_{u}^{0}(0)\right] \Pi_{u}^{1}}{4\left[Z_{u}+\Pi_{u}^{0}(0)\right]\left[Z_{q}+\Pi_{q}^{0}(0)\right]} \\
\frac{y_{b}^{(0)}}{m_{b}^{(0)}}-\frac{F_{b}}{s_{h} f_{h}} \simeq \frac{s_{h}}{f_{h}} \frac{2\left|m_{d}^{1}(0)\right|\left|m_{d}^{1}(0)\right|^{\prime}-\left[Z_{q}+\Pi_{q}^{0}(0)\right] \Pi_{d}^{1}}{4\left[Z_{d}+\Pi_{d}^{0}(0)\right]\left[Z_{q}+\Pi_{q}^{0}(0)\right]}
\end{aligned}
$$

$y_{b} / m_{b}$ in this model is exactly as in the $\mathrm{MCHM}_{5-5-10}$ when expressed in terms of the correlators, although the correlators themselves are different in both models. This can be understood, again, because the bottom mass arises from the coupling between $q$ and $d$, which share the same embedding in both models. Eq. (3.35) shows that the $\mathcal{O}\left(s_{h}^{2}\right)$ corrections to $y_{b}^{(0)} / m_{b}^{(0)}$ in this model is also suppressed by $s_{d}^{2} \ll 1$.

\section{6 $\mathrm{MCHM}_{14-14-10}$}

In this model: $Q, U \sim \mathbf{1 4}_{2 / 3}$ and $D \sim \mathbf{1 0}_{2 / 3}$. In unitary gauge the Yukawa term of the fermion Lagrangian (3.1) includes:

$$
\mathcal{L}_{y} \supset y_{u} \Phi^{\dagger} \bar{Q}_{L} U_{R} \Phi+y_{d} \Phi^{\dagger} \bar{Q}_{L} D_{R} \Phi
$$

The following term is also allowed by the symmetries

$$
\mathcal{L}_{y} \supset \tilde{y}_{u}\left(\Phi^{\dagger} \bar{Q}_{L} \Phi\right)\left(\Phi^{\dagger} U_{R} \Phi\right)
$$

having potentially important consequences for the phenomenology, as will be discussed in the next section.

The correlators of the effective Lagrangian (3.3) are:

$$
\begin{aligned}
\Pi_{u_{L}} & =\Pi_{q}^{0}+\Pi_{q}^{1}\left(\frac{c_{h}^{2}}{2}+\frac{s_{h}^{2}}{4}\right)+\Pi_{q}^{2} s_{h}^{2} c_{h}^{2}, & \Pi_{d_{L}} & =\Pi_{q}^{0}+\Pi_{q}^{1} \frac{c_{h}^{2}}{2}, \\
\Pi_{u_{R}} & =\Pi_{u}^{0}+\Pi_{u}^{1}\left(\frac{4}{5} c_{h}^{2}+\frac{s_{h}^{2}}{20}\right)+\Pi_{u}^{2} \frac{\left(4 c_{h}^{2}-s_{h}^{2}\right)^{2}}{20}, & \Pi_{d_{R}} & =\Pi_{d}^{0}+\Pi_{d}^{1} \frac{s_{h}^{2}}{4} \\
M_{u} & =i m_{u}^{1} \frac{3}{4 \sqrt{5}} s_{h} c_{h}+i m_{u}^{2} \frac{1}{2 \sqrt{5}} s_{h} c_{h}\left(4 c_{h}^{2}-s_{h}^{2}\right), & M_{d} & =i m_{d}^{1} \frac{s_{h} c_{h}}{2 \sqrt{2}}
\end{aligned}
$$

where the $\Pi_{\psi}^{i}$ are defined in analogy to eqs. (3.11) and (3.17), with the $\Phi$-dependent terms following the structure displayed in eqs. (3.36) and (3.37) for the Yukawa terms in this model [see also comments following eq. (3.11)]. Since a 14 of SO(5) decomposes under 
$\mathrm{SO}(4)$ as $\mathbf{1 4} \sim \mathbf{1}+\mathbf{4}+\mathbf{9}$, we find

$$
\begin{array}{rlrl}
\Pi_{q}^{0} & =\hat{\Pi}_{q^{(9)}}, & \Pi_{u}^{0} & =\hat{\Pi}_{u^{(9)}}, \\
\Pi_{d}^{0} & =\hat{\Pi}_{d^{(6)}}, & & \Pi_{u}^{1}=2\left(\hat{\Pi}_{u^{(4)}}-\hat{\Pi}_{u^{(9)}}\right), \\
\Pi_{q}^{1} & =2\left(\hat{\Pi}_{q^{(4)}}-\hat{\Pi}_{q^{(9)}}\right), & & \\
\Pi_{d}^{1} & =2\left(\hat{\Pi}_{d^{(4)}}-\hat{\Pi}_{d^{(6)}}\right), & \Pi_{u}^{2} & =\frac{1}{4}\left(5 \hat{\Pi}_{u^{(1)}}-8 \hat{\Pi}_{u^{(4)}}+3 \hat{\Pi}_{u^{(9)}}\right), \\
\Pi_{q}^{2} & =\frac{1}{4}\left(5 \hat{\Pi}_{q^{(1)}}-8 \hat{\Pi}_{q^{(4)}}+3 \hat{\Pi}_{q^{(9)}}\right), & m_{d}^{0} & =0, \\
m_{u}^{0} & =\hat{M}_{u^{(1)}}, & m_{d}^{1} & =2 i \hat{M}_{d^{(4)}}, \\
m_{u}^{1} & =2\left(\hat{M}_{u^{(4)}}-\hat{M}_{u^{(9)}}\right), & & \\
m_{u}^{2} & =\frac{1}{4}\left(5 \hat{M}_{u^{(1)}}-8 \hat{M}_{u^{(4)}}+3 \hat{M}_{u^{(9)}}\right) . &
\end{array}
$$

where the hatted correlators are given in appendix C.7.

The prediction for $y_{\psi} / m_{\psi}$ is:

$$
\begin{aligned}
\frac{y_{t}^{(0)}}{m_{t}^{(0)}}-\frac{F_{t}}{s_{h} f_{h}} \simeq & \frac{s_{h}}{f_{h}}\left\{-2 \frac{-3\left|m_{u}^{1}(0)\right|\left[\Pi_{q}^{1}(0)-4 \Pi_{q}^{2}(0)\right]+16\left|m_{u}^{2}(0)\right|\left[5 Z_{q}+5 \Pi_{q}^{0}(0)+2 \Pi_{q}^{1}(0)+2 \Pi_{q}^{2}(0)\right]}{\left[3\left|m_{u}^{1}(0)\right|+8\left|m_{u}^{2}(0)\right|\right]\left[2 Z_{q}+2 \Pi_{q}^{0}(0)+\Pi_{q}^{1}(0)\right]}\right. \\
& \left.+\frac{-\left[3\left|m_{u}^{1}(0)\right|+8\left|m_{u}^{2}(0)\right|\right]\left[3\left|m_{u}^{1}(0)\right|^{\prime}+8\left|m_{u}^{2}(0)\right|^{\prime}\right]+5\left[2 Z_{q}+2 \Pi_{q}^{0}(0)+\Pi_{q}^{1}(0)\right]\left[3 \Pi_{u}^{1}(0)+8 \Pi_{u}^{2}(0)\right]}{\left[5 Z_{u}+5 \Pi_{u}^{0}(0)+4 \Pi_{u}^{1}(0)+4 \Pi_{u}^{2}(0)\right]\left[2 Z_{q}+2 \Pi_{q}^{0}(0)+\Pi_{q}^{1}(0)\right]}\right\},
\end{aligned}
$$

$$
\frac{y_{b}^{(0)}}{m_{b}^{(0)}}-\frac{F_{b}}{s_{h} f_{h}} \simeq \frac{s_{h}}{f_{h}} \frac{2\left|m_{d}^{1}(0)\right|\left|m_{d}^{1}(0)\right|^{\prime}+4\left[Z_{d}+\Pi_{d}^{0}(0)\right] \Pi_{q}^{1}-\left[2 Z_{q}+2 \Pi_{q}^{0}(0)+\Pi_{q}^{1}(0)\right] \Pi_{d}^{1}}{4\left[Z_{d}+\Pi_{d}^{0}(0)\right]\left[2 Z_{q}+2 \Pi_{q}^{0}(0)+\Pi_{q}^{1}(0)\right]} .
$$

$y_{b} / m_{b}$ in this model is exactly as in the $\mathrm{MCHM}_{10}$ when expressed in terms of the correlators, although the correlators themselves are different in both models.

\section{$3.7 \quad \mathrm{MCHM}_{14-1-10}$}

In this model: $Q \sim \mathbf{1 4}_{2 / 3}, U \sim \mathbf{1}_{2 / 3}$ and $D \sim \mathbf{1 0}_{2 / 3}$ : In unitary gauge the Yukawa term of the fermion Lagrangian (3.1) reads:

$$
\mathcal{L}_{y}=y_{u}\left(\Phi^{\dagger} \bar{Q}_{L} \Phi\right) U_{R}+y_{d} \Phi^{\dagger} \bar{Q}_{L} D_{R} \Phi .
$$

The correlators of the effective Lagrangian (3.3) are:

$$
\begin{aligned}
\Pi_{u_{L}} & =\Pi_{q}^{0}+\Pi_{q}^{1}\left(\frac{c_{h}^{2}}{2}+\frac{s_{h}^{2}}{4}\right)+\Pi_{q}^{2} c_{h}^{2} s_{h}^{2}, & \Pi_{d_{L}} & =\Pi_{q}^{0}+\Pi_{q}^{1} \frac{c_{h}^{2}}{2}, \\
\Pi_{u_{R}} & =\Pi_{u}^{0}, & \Pi_{d_{R}} & =\Pi_{d}^{0}+\Pi_{d}^{1} \frac{s_{h}^{2}}{4}, \\
M_{u} & =-m_{u}^{1} \frac{s_{h}}{2}, & M_{d} & =-m_{d}^{1} \frac{s_{h} c_{h}}{2 \sqrt{2}} .
\end{aligned}
$$


where the $\Pi_{\psi}^{i}$ are defined in analogy to eqs. (3.11) and (3.17), with the $\Phi$-dependent terms following the structure displayed in eq. (3.42) for the Yukawa terms in this model [see also comments following eq. (3.11)]. Using the previous decompositions of $\mathbf{1 4}$ and $\mathbf{1 0}$ of $\mathrm{SO}(5)$ under $\mathrm{SO}(4)$ :

$$
\begin{array}{rlrl}
\Pi_{q}^{0} & =\hat{\Pi}_{q^{(9)}}, & \Pi_{u}^{0}=\hat{\Pi}_{u^{(1)}}, & \Pi_{d}^{0}=\hat{\Pi}_{d^{(6)}}, \\
\Pi_{q}^{1}=2\left(\hat{\Pi}_{q^{(4)}}-\hat{\Pi}_{q^{(9)}}\right), & \Pi_{d}^{1}=2\left(\hat{\Pi}_{d^{(4)}}-\hat{\Pi}_{d^{(6)}}\right), \\
\Pi_{q}^{2}=\frac{1}{4}\left(5 \hat{\Pi}_{q^{(1)}}-8 \hat{\Pi}_{q^{(4)}}+3 \hat{\Pi}_{q^{(9)}}\right), & & \\
m_{u}^{0} & =0, & m_{d}^{0}=0, & \\
m_{u}^{1} & =\frac{\sqrt{5}}{2} \hat{M}_{u^{(1)}}, & m_{d}^{1} & =2 i \hat{M}_{d^{(4)}} .
\end{array}
$$

where the hatted correlators are given in appendix C.6.

The prediction for $y_{\psi} / m_{\psi}$ is:

$$
\begin{aligned}
& \frac{y_{t}^{(0)}}{m_{t}^{(0)}}-\frac{F_{t}}{s_{h} f_{h}} \simeq \frac{s_{h}}{f_{h}} \frac{-8\left|m_{u}^{1}(0)\right|\left|m_{u}^{1}(0)\right|^{\prime}+\left[Z_{u}+\Pi_{u}^{0}(0)\right]\left[\Pi_{q}^{1}(0)-4 \Pi_{q}^{2}(0)\right]}{2\left[Z_{u}+\Pi_{u}^{0}(0)\right]\left[2 Z_{q}+2 \Pi_{q}^{0}(0)+\Pi_{q}^{1}(0)\right]} ; \\
& \frac{y_{b}^{(0)}}{m_{b}^{(0)}}-\frac{F_{b}}{s_{h} f_{h}} \simeq \frac{s_{h}}{f_{h}} \frac{2\left|m_{d}^{1}(0)\right|\left|m_{d}^{1}(0)\right|^{\prime}+4\left[Z_{d}+\Pi_{d}^{0}(0)\right] \Pi_{q}^{1}-\left[2 Z_{q}+2 \Pi_{q}^{0}(0)+\Pi_{q}^{1}(0)\right] \Pi_{d}^{1}}{4\left[Z_{d}+\Pi_{d}^{0}(0)\right]\left[2 Z_{q}+2 \Pi_{q}^{0}(0)+\Pi_{q}^{1}(0)\right]} .
\end{aligned}
$$

The prediction for $y_{b} / m_{b}$ in this model is exactly as in the $\mathrm{MCHM}_{10}$ when expressed in terms of the correlators, although the correlators themselves are different in both models.

\subsection{Other models based on the lowest-dimensional reps. of $\mathrm{SO}(5)$}

Although we will not provide all the details, we list here the other possible models one can consider when using the $\mathbf{1}, \mathbf{5}, \mathbf{1 0}$ and $\mathbf{1 4}$ representations of $\mathrm{SO}(5)$ in all possible combinations for the quark sector (assuming the same assignments for all the families). Besides the cases given above, one can have an $\mathrm{MCHM}_{5-1-10}, \mathrm{MCHM}_{14-10-10}, \mathrm{MCHM}_{10-14-10}$, $\mathrm{MCHM}_{14-5-10}$ and $\mathrm{MCHM}_{5-14-10}$. This would exhaust all the models that allow to write Yukawa couplings (in particular for the top quark, which is hard to imagine arising from other than tree-level effects). For instance, the $\mathrm{MCHM}_{10-1-X}$ does not allow to write the operator $y_{u}\left(\Phi^{\dagger} \bar{Q}_{L} \Phi\right) U_{R}+$ h.c. since it vanishes due to the antisymmetry of the $\mathbf{1 0}$. Some of these models (the $\mathrm{MCHM}_{14-5-10}$ and $\mathrm{MCHM}_{5-14-10}$ ), like the $\mathrm{MCHM}_{14-14-10}$ described in detail in section 3.6, allow for two Yukawa structures in the up sector, which can a priori lead to qualitative differences with the remaining models that allow only a single Yukawa structure. We will study in detail only the $\mathrm{MCHM}_{14-14-10}$ to illustrate the possible features in such cases, and will restrict our comments for the models mentioned in this subsection to only a few general remarks in the following sections (but enough to get a feel for their phenomenology). 


\section{Corrections to low-energy observables in the MCHM}

To analyze the low-energy consequences of the model one can either diagonalize the gauge and fermion mass matrices, explicitly including the heavy states and their mixing with the elementary fields. The SM fields are then identified as the lowest lying states in the presence of a given $\langle h\rangle$. The latter is actually determined dynamically as discussed in section 5 , but the procedure works for any fixed vev. Finding the Higgs mass, however, requires the minimization of the potential, and incorporating this information will be deferred to later sections.

Alternatively, one can obtain an effective theory for the fields on site- 0 , as done in section 2.4 for the gauge fields and in section 3 for the fermion sector. The zeroes of the correlators thus obtained determine the spectrum of the model. The correlators also encode in their Higgs vev dependence information regarding the couplings of the physical fields and the Higgs boson, as discussed in the previous section.

Although the numerical analysis to be presented in section 6 has been obtained by the previous methods (and we have checked that they agree), it is useful to have a simple analytic approximation that captures the main phenomenological features of the Higgs sector in composite Higgs models. To do so, one starts from the following relation that holds in the simplest situations, which includes most of the models we study:

$$
\sum_{n} \frac{y_{\psi}^{(n)}}{m_{\psi}^{(n)}}=\frac{1}{2} \frac{d}{d h} \log \operatorname{det}\left(M_{\psi}^{\dagger} M_{\psi}\right)=\frac{1}{s_{h} f_{h}} F_{\psi}\left(s_{h}\right),
$$

where $m_{\psi}^{(n)}$ and $y_{\psi}^{(n)}$ are the mass and the Yukawa coupling of the $\mathrm{n}$-th fermionic resonance to the Higgs, respectively, and $M_{\psi}$ is the $h$-dependent mass matrix. The fact that the above trace depends only on $s_{h}=\sin \left(h / f_{h}\right)$, but not on other parameters of the model ${ }^{10}$ is not a general statement, but a consequence of the particular models considered in this work. In the simplest situation there is just one Yukawa term that leads only to one non-trivial $\mathrm{SO}(4)$ invariant for each sector, resulting in a determinant that factorizes as $\operatorname{det}\left(M_{\psi}^{\dagger} M_{\psi}\right)=\hat{F}_{\psi}\left(s_{h}\right) h_{\psi}(y, \Delta, m)$. Therefore, its logarithmic derivative depends only on $s_{h}$ and $f_{h} . F_{\psi}\left(s_{h}\right)$ is a model-dependent function that depends on the representation of the fermions under $G_{1}[19,35,36]$.

In the general situation, for arbitrary representations of the composite fermions, there is more than one non-trivial $\mathrm{SO}(4)$ invariant arising from the Yukawa interactions in each sector. The determinant does not factorize in this case and its derivative generically depends on other microscopic parameters as well, such as the composite Yukawa couplings. This is the case for the most general $\mathrm{MCHM}_{14-14-10}$ discussed in section 3.6. This could be important for the phenomenology, since in the general case one could in principle obtain enhancement or suppression of the gluon fusion process in different regions of the parameter space, while there is no such freedom for the minimal cases with just one invariant.

\footnotetext{
${ }^{10}$ However, one should remember that $\langle h\rangle$ itself is determined by the effective potential, which is calculable and depends on various microscopic parameters. Therefore, the most precise statement is that the r.h.s. of eq. (4.1) depends on the microscopic parameters only through $h / f_{h}$.
} 
Under the assumption that eq. (4.1) holds, the additional useful observation is that, to leading order in $\epsilon=\sin \left(v / f_{h}\right)$, the sum is saturated by the zero-mode term, leading to

$$
\frac{y_{\psi}^{(0)}}{m_{\psi}^{(0)}} \approx \frac{1}{\epsilon f_{h}}\left[F_{\psi}(\epsilon)+\mathcal{O}\left(\epsilon^{2} s_{\psi_{L}}^{2}\right)+\mathcal{O}\left(\epsilon^{2} s_{\psi_{R}}^{2}\right)\right]
$$

where $s_{\psi_{L}}$ and $s_{\psi_{R}}$ are the LH and RH elementary-composite mixing angles, respectively. This was explicitly shown in section 3 for each model, and in section 6 we will further show numerically that the above approximation works reasonably well even in the top sector (we will also discuss the cases where important deviations arise).

Except for the case considered in section 3.6 and two embeddings described in section 3.8, we find only two different functions for the models considered in this work:

$$
F_{1}=\frac{1-2 \epsilon^{2}}{\sqrt{1-\epsilon^{2}}}, \quad F_{2}=\sqrt{1-\epsilon^{2}}
$$

The $\mathrm{MCHM}_{14-14-10}$ presented in section 3.6 is somewhat different in that two different Yukawa structures are allowed [see eqs. (3.36) and (3.37)]. As a result, the trace involves a function with a non-trivial dependence on these Yukawa couplings, not just on $\epsilon$ :

$$
\frac{1}{\epsilon f_{h}} F_{3} \equiv \operatorname{tr}\left(Y_{u} M_{u}^{-1}\right)=\frac{1}{\epsilon f_{h}} \frac{\left(6 \epsilon^{2}-3\right) y_{u}-2\left(20 \epsilon^{4}-23 \epsilon^{2}+4\right) \tilde{y}_{u}}{\sqrt{1-\epsilon^{2}}\left(2\left(5 \epsilon^{2}-4\right) \tilde{y}_{u}-3 y_{u}\right)},
$$

which can change the size and sign of $F_{3}$. Being $F_{3}$ a homogeneous function of the Yukawa couplings, it depends only on the ratio $r_{y}=\tilde{y}_{u} / y_{u}$. For $r_{y}=0$ one recovers the $F_{1}$ function of the other models: $\left.F_{3}\right|_{r_{y}=0}=F_{1}$. In the opposite limit we define a new function

$$
\tilde{F}_{3} \equiv \lim _{r_{y} \rightarrow \infty} F_{3}=\frac{4-23 \epsilon^{2}+20 \epsilon^{4}}{\sqrt{1-\epsilon^{2}}\left(4-5 \epsilon^{2}\right)}
$$

For $r_{y} \rightarrow \infty$ one can obtain in principle a large suppression, since $\tilde{F}_{3}$ changes sign for $\epsilon \simeq 0.46 . F_{3}$ interpolates between $F_{1}$ and $\tilde{F}_{3}$ as $r_{y}$ varies, thus one can expect a suppression larger than $F_{1}$ in the general case (see right panel of figure 3 ). However there is a small region of the parameter space where there could be an enhancement and a violent change of sign of $F_{3}$, as a consequence of an accidental cancellation in $\operatorname{det} M_{u}$ that leads to a singularity of $F_{3}$ (this has also been observed in ref. [21]). This is connected to the existence of a very light resonance in this region. For $\epsilon \in(0,0.5)$ the singularity is present if $r_{y} \in$ $(-6 / 11,-3 / 8)$, thus for points of the parameter space near the singularity the value of $F_{3}$ can be very large, changing sign across the singularity. Although a large correction in any direction is possible in this model it requires tuning of the Yukawa couplings. This large correction, being associated with a zero of $\operatorname{det} M_{u}$, signals the presence of a very light mode in the spectrum, that can be in conflict with bounds on top partners. Moreover, by performing a random scan we have checked that the points able to reproduce the spectrum and EW constraints are usually far from the singularity. Thus, we typically obtain a suppression as opposed to an enhancement from this more complicated function. 


\begin{tabular}{|c|c|c|c|c|c|c|c|}
\hline$r /$ MCHM & $10-5-10$ & $5-5-10$ & $\begin{array}{c}5-10-10, \\
5-1-10\end{array}$ & $\begin{array}{c}14-1-10 \\
14-10-10 \\
10-14-10\end{array}$ & $14-14-10$ & $14-5-10$ & $5-14-10$ \\
\hline$r_{t}$ & $F_{2}$ & $F_{1}$ & $F_{2}$ & $F_{1}$ & $F_{3}$ & $F_{4}$ & $F_{5}$ \\
\hline$r_{b}$ & $F_{1}$ & $F_{2}$ & $F_{2}$ & $F_{1}$ & $F_{1}$ & $F_{1}$ & $F_{1}$ \\
\hline$r_{V}$ & $F_{2}$ & $F_{2}$ & $F_{2}$ & $F_{2}$ & $F_{2}$ & $F_{2}$ & $F_{2}$ \\
\hline$r_{g}$ & $F_{2}$ & $F_{1}$ & $F_{2}$ & $F_{1}$ & $F_{3}$ & $F_{4}$ & $F_{5}$ \\
\hline
\end{tabular}

Table 1. Ratio of Higgs SM and MCHM couplings, $r=c^{M C H M} / c^{S M}$, approximated by the functions $F_{i} . g$ stands for the loop induced gluon coupling (we have only considered the top sector effect for $r_{g}$ in this table, but in the numerical results we have included the bottom sector as well), $\psi=t, b$ are the Yukawa couplings and $V=W, Z$ is the coupling to the massive EW gauge bosons. For completeness, we include also the result for additional models that were not described in full detail in the main text.

Another important consequence is that the presence of two different flavor structures leads to missalignement of Higgs coupling in LR operators [38]. For anarchic models, these new sources of flavor violation mediated by Higgs exchange are too large compared with bounds from flavor physics, requiring extra protection. For this reason we will perform one scan imposing $\tilde{y}_{u}=0$, and a second one allowing $\tilde{y}_{u} \neq 0$. It turns out that the latter ends up preferring regions with $y_{u} \ll \tilde{y}_{u}$, so that it is effectively described by $\tilde{F}_{3}(\epsilon)$ given in eq. (4.5) above.

The other models mentioned in section 3.8 can be described by the same $F_{i}(\epsilon)$ above, except for the $\mathrm{MCHM}_{14-5-10}$ and $\mathrm{MCHM}_{5-14-10}$ which lead to the following new functions that, like the one for the $\mathrm{MCHM}_{14-14-10}$, also depend on the microscopic Yukawa couplings $\left[F_{4}\right.$ and $F_{5}$ are defined in analogy to eq. (4.4)]:

$$
F_{4}=\frac{\sqrt{1-\epsilon^{2}}\left(y_{u}+2 \tilde{y}_{u}-6 \tilde{y}_{u} \epsilon^{2}\right)}{y_{u}+2 \tilde{y}_{u}\left(1-\epsilon^{2}\right)}, \quad F_{5}=\frac{\sqrt{1-\epsilon^{2}}\left(y_{u}-\tilde{y}_{u}\left(4-15 \epsilon^{2}\right)\right)}{y_{u}-\tilde{y}_{u}\left(4-5 \epsilon^{2}\right)} .
$$

In the limiting cases where only one of the two Yukawa couplings is turned on, the above become functions of $\epsilon$ only. In such limits, they lie between the curves for $F_{1}$ and $\tilde{F}_{3}$ in the right panel of figure 3 in section 6.2 (they are not shown in the figure).

The $F_{i}$ functions defined in eqs. (4.3)-(4.6) encode the deviations from the SM couplings, $r=c_{\mathrm{MCHM}} / c_{\mathrm{SM}}$, as shown in table $1,{ }^{11}$ and determine the $c_{i}$ coefficients of the following set of operators in the low-energy theory:

$$
\begin{array}{ll}
\mathcal{O}_{g}=h G_{\mu \nu}^{a} G^{a \mu \nu}, & \mathcal{O}_{\gamma}=h A_{\mu \nu} A^{\mu \nu}, \\
\mathcal{O}_{w}=h W_{\mu}^{+} W^{-\mu}, & \mathcal{O}_{z \gamma}=h Z_{\mu} Z^{\mu},
\end{array}
$$

\footnotetext{
${ }^{11}$ Some of these functions have been shown previously in refs. [33] and [21].
} 
These are the leading order operators involved in Higgs production and decay at the LHC. Since the operators $\mathcal{O}_{g}$ and $\mathcal{O}_{\gamma}$ break the shift symmetry of the pNGB Higgs and must, therefore, involve the explicit symmetry breaking parameters such as the SM gauge and Yukawa couplings, they are generated at loop level. Our computation gives the contributions to the Wilson coefficients of these operators in the MCHM after EWSB to all order in the Higgs vev, leading to coefficients $c_{\mathcal{O}}(v / f)$. Expanding these coefficients in powers of $v / f$ one can do the matching to the Wilson coefficients of dimension-six operators which, in the basis of refs. [39-41], are

$$
\begin{aligned}
\mathcal{O}_{H} & =\frac{1}{2}\left(\partial_{\mu}|H|^{2}\right)^{2}, & \mathcal{O}_{y_{f}} & =|H|^{2} \bar{q}_{L} H f_{R}, \\
\mathcal{O}_{G G} & =|H|^{2} G_{\mu \nu} G^{\mu \nu}, & \mathcal{O}_{B B} & =|H|^{2} B_{\mu \nu} B^{\mu \nu}, \\
\mathcal{O}_{W} & =\frac{i}{2}\left(H^{\dagger} \sigma^{a} \overleftrightarrow{D}_{\mu} H\right) D^{\nu} W_{\mu \nu}^{a}, & \mathcal{O}_{B} & =\frac{i}{2}\left(H^{\dagger} \overleftrightarrow{D}_{\mu} H\right) \partial^{\nu} B_{\mu \nu}, \\
\mathcal{O}_{H W} & =i\left(D^{\mu} H\right)^{\dagger} \sigma^{a}\left(D^{\nu} H\right) W_{\mu \nu}^{a}, & \mathcal{O}_{H B} & =i\left(D^{\mu} H\right)^{\dagger}\left(D^{\nu} H\right) B_{\mu \nu}
\end{aligned}
$$

By redefining the Higgs field one can show that $\mathcal{O}_{H}$ renormalizes the Higgs couplings to all the other SM fields. $\mathcal{O}_{G G}, \mathcal{O}_{B B}$ and $\mathcal{O}_{-}=\left(\mathcal{O}_{W}-\mathcal{O}_{B}\right)-\left(\mathcal{O}_{H W}-\mathcal{O}_{H B}\right)$ enter in the interactions $h g g, h \gamma \gamma$ and $h Z \gamma$, respectively, and $\mathcal{O}_{y_{f}}$ enters in $h f \bar{f}$ [9]. The Wilson coefficients $c_{H}, c_{W}$ and $c_{B}$ are universal for all the MCHM with $\mathrm{SO}(5) / \mathrm{SO}(4)$ breaking and have been computed in the SILH description [9]:

$$
c_{H}=1 ; \quad c_{W}=c_{B}=\frac{27 \pi^{2}}{256} \simeq 1.0 .
$$

$c_{y}$ has been computed in [9] for the top sector in the $\mathrm{MCHM}_{5}$. In general it can be obtained from the functions $F_{\psi}$ that codify the deviation of the Yukawa coupling, leading to:

$$
\begin{array}{ll}
c_{y_{t}}=1, & \text { for the } \mathrm{MCHM}_{5,10,14-14-10,14-1-10,5-5-10}, \\
c_{y_{t}}=0, & \text { for the } \mathrm{MCHM}_{10-5-10,5-10-10} \\
c_{y_{b}}=1, & \text { for the } \mathrm{MCHM}_{5,10,14-14-10,14-1-10,10-5-10} \\
c_{y_{b}}=0, & \text { for the } \mathrm{MCHM}_{5-5-10,5-10-10} .
\end{array}
$$

The coefficients $c_{g, \gamma}$ and $c_{H W, H B}$ are generated at loop level. Starting with $\mathcal{O}_{g}$, this operator is generated by fermion loops. For each fermion species there is a contribution (see appendix D)

$$
c_{g} \propto \sum_{n} \frac{y_{n}}{m_{n}} A_{1 / 2}\left(\tau_{n}\right), \quad \tau_{n}=\frac{m_{h}^{2}}{4 m_{n}^{2}} .
$$

For heavy fermions, $\left.A_{1 / 2}(\tau)\right|_{\tau \rightarrow 0} \rightarrow 4 / 3$. Thus, considering heavy resonances we obtain:

$$
c_{g} \propto \frac{4}{3}\left[\operatorname{tr}\left(Y_{\psi} M_{\psi}^{-1}\right)-\frac{y_{\psi}^{(0)}}{m_{\psi}^{(0)}}\right]+\frac{y_{\psi}^{(0)}}{m_{\psi}^{(0)}} A_{1 / 2}\left(\tau_{0}\right),
$$

with the index 0 referring to the would-be 0-mode, associated with the SM mass eigenstate. The last term is similar to the SM one, up to corrections in the Yukawa coupling. These 
corrections are important only if the mixing is large. Since $A_{1 / 2}(\tau) \rightarrow_{\tau \rightarrow \infty} 0$, this term is small for light fermions, $m_{\psi} \ll m_{h}$. As was shown in section 3 , the first term is also small if the mixing of both, the Left and Right chiralities, is small. For the top quark one can take the limit $A_{1 / 2}\left(\tau_{t}\right) \rightarrow 4 / 3$, and eq. (4.14) is dominated by $4 / 3 \operatorname{tr}\left(Y_{t} M_{t}^{-1}\right)$, which is the sum considered in eq. (4.1). Thus, one can also obtain an approximate expression for the gluon fusion process in terms of the functions above, as shown in table 1. For the coupling of the Higgs to two photons, there is an additional contribution due to the heavy spin-1 resonances. However, a similar sum rule applies which allows to obtain an approximate analytical expression. These will be studied in more detail in section 6 , after taking into account the constraints from the recently measured Higgs mass [42], as well as the masses of the $Z$ gauge boson and the top and bottom quarks, which have the most important impact on the Higgs potential and the Higgs phenomenology.

\section{Higgs potential}

Discrete models of pNGB Higgs can lead to a finite Higgs potential under some suitable assumptions. The degree of divergence of the Higgs potential depends on the particular mechanism of collective breaking, being thus model dependent. There are at least two concepts involved: distance between the sites where the symmetries protecting the pNGB potential are broken, and number of symmetries broken on each site.

The Higgs potential can be computed by the holographic method

$$
\begin{aligned}
V(h)=\int \frac{d^{4} p}{(2 \pi)^{4}}\left[\frac{3}{2} \sum_{i=1}^{2} \log \Pi_{w_{L}^{i}}\right. & +\frac{3}{2} \log \left[\Pi_{w_{L}^{3}} \Pi_{b}-\left(\Pi_{w_{L}^{3} b}\right)^{2}\right] \\
& \left.-2 N_{c} \sum_{\psi} \log \left[p^{2} \Pi_{\psi_{L}} \Pi_{\psi_{R}}-\left|M_{\psi}\right|^{2}\right]\right],
\end{aligned}
$$

where the correlators are obtained from sections 2.4 and 3, taking care to add the "bare" kinetic terms, as in eqs. (2.7) and (3.3), which were not included as part of the definition of the correlators in those sections:

$$
\Pi_{w_{L}^{i}}=\frac{p^{2}}{g_{0}^{2}}+\Pi_{\tilde{w}_{L}^{i}}, \quad \Pi_{w_{L}^{3} b}=\Pi_{\tilde{w}_{L}^{3} \tilde{b}}, \quad \Pi_{b}=\frac{p^{2}}{g_{0}^{\prime 2}}+\Pi_{\tilde{b}},
$$

and similarly for the fermionic correlators. Equivalently, one can use the standard expression for the Coleman-Weinberg potential in terms of determinants involving the Higgsdependent mass matrices of the gauge and fermion fields. We have checked that the same results can be reproduced with either approach. Note that eq. (5.1) contains the photon, although it does not contribute to the Higgs potential, and one can regularize the divergent constant terms by subtracting $V(0)$.

\subsection{Finiteness of the 1-loop Higgs potential}

In this subsection we illustrate in a toy example how the inclusion/exclusion of certain operators in the Lagrangian affects the divergence structure of the Higgs potential. Our 
example is based on the fundamental representation of $\mathrm{SO}(5)$, but the conclusion holds for other representations as well. In order to understand the structure of divergences of the $h$-dependent terms, let us consider the 2 -site model with the following set of fields:

- site 0: an elementary fermion doublet $q_{L}$ and a singlet $t_{R}$ of a global symmetry $G_{0}=\mathrm{SU}(2)_{L} \cdot{ }^{12}$

- site 1: four chiral composite fermions $Q_{L}, Q_{R}, T_{L}, T_{R}$, each transforming in the fundamental representation of a different global SO(5), called: $G_{Q_{L}}, G_{Q_{R}}, G_{T_{L}}, G_{T_{R}}$. In this site there is also a scalar $\Phi_{1}$ transforming in the fundamental of another $\mathrm{SO}(5)$, called: $G_{1}$. The vev of $\Phi_{1}$ spontaneously breaks $G_{1}$ to $\mathcal{H}_{1}=\operatorname{SO}(4)$.

Notice that before introducing fermion masses, each chiral fermion of the composite sector transforms independently, leading to a large global symmetry (in fact, the symmetry is much larger, but we need only focus on this subgroup). The Higgs, being a NGB, is in the coset $G_{1} / \mathcal{H}_{1}$. The following operators break different symmetries:

- $m_{Q} \bar{Q} Q: G_{Q_{L}} \times G_{Q_{R}} \rightarrow G_{Q_{L+R}}=\mathrm{SO}(5)$,

- $m_{T} \bar{T} T: G_{T_{L}} \times G_{T_{R}} \rightarrow G_{T_{L+R}}=\mathrm{SO}(5)$,

- $\Delta_{q} \bar{q}_{L} Q_{R}+$ h.c.: $G_{0} \times G_{Q_{R}} \rightarrow G_{Q_{R}+0}=\mathrm{SU}(2)$,

- $\Delta_{t} \bar{t}_{R} T_{L}+$ h.c.: $G_{0} \times G_{T_{L}} \rightarrow G_{T_{L}+0}=\mathrm{SU}(2)$,

- $y_{T} \bar{Q}_{L} \Phi_{1} \Phi_{1}^{\dagger} T_{R}+$ h.c.: $G_{Q_{L}} \times G_{T_{R}} \times G_{1} \rightarrow G_{Q_{L}+T_{R}+1}=\operatorname{SO}(5)$,

- $y_{T}^{\prime} \bar{Q}_{R} \Phi_{1} \Phi_{1}^{\dagger} T_{L}+$ h.c.: $G_{Q_{R}} \times G_{T_{L}} \times G_{1} \rightarrow G_{Q_{R}+T_{L}+1}=\operatorname{SO}(5)$.

There is some abuse of notation in the previous paragraph, since $G_{Q_{R}, T_{L}}$ and $G_{0}$ have different dimensions, so that when writing $G_{T_{L}+0}$ we really mean the diagonal subgroup $G_{0}^{\prime}=\mathrm{SU}(2)$. In addition to the above, the symmetries allow operators of the form $\bar{Q}_{L} \Phi_{1} \Phi_{1}^{\dagger} Q_{R}+$ h.c. or $\bar{T}_{L} \Phi_{1} \Phi_{1}^{\dagger} T_{R}+$ h.c., which would also lead to divergences in the Higgs potential of the 2-site model. With three or more sites, these would lead to a finite 1loop result $[28,29]$. For illustration, we limit the following discussion to the operators listed above.

A Higgs potential requires insertions of $y_{T}$ and/or $y_{T}^{\prime}$. Let us consider the following cases:

(a) $y_{T}^{\prime}=0$ : the $y_{T}$ term only preserves the diagonal subgroup $G_{Q_{L}+T_{R}+1}$. The Higgs is in the coset $G_{Q_{L}+T_{R}+1} / \mathcal{H}_{1}$, and thus a Higgs potential requires explicit breaking of $G_{Q_{L}+T_{R}+1}$. This necessitates interactions with the elementary sector, which arise from the $\Delta_{q}$ and/or $\Delta_{t}$ terms. However, due to their chirality structure, insertions of $\Delta_{q, t}$ still do not break $G_{Q_{L}+T_{R}+1}: G_{Q_{L}+T_{R}+1} \times G_{0}$ is broken only after additional $m_{Q, T}$ insertions. Thus,

$$
V_{H} \sim\left(\Delta_{q, t} m_{Q, T} y_{T}\right)^{2} .
$$

\footnotetext{
${ }^{12}$ For simplicity we ignore $\mathrm{U}(1)_{Y}$ in this discussion.
} 
(b) $y_{T}=0$ : the $y_{T}^{\prime}$ term only preserves the diagonal subgroup $G_{Q_{R}+T_{L}+1}$ and the Higgs is in the coset $G_{Q_{R}+T_{L}+1} / \mathcal{H}_{1}$. In this case, insertions of $\Delta_{q}$ and/or $\Delta_{t}$ break $G_{Q_{R}+T_{L}+1} \times G_{0}$ without the need of $m_{Q, T}$ insertions:

$$
V_{H} \sim\left(\Delta_{q, t} y_{T}^{\prime}\right)^{2}
$$

The previous arguments show how the dimension of the operators leading to $V_{H}$ depends on the presence of $y_{T}^{\prime}$, leading to logarithmic divergences at 1-loop for $y_{T}^{\prime} \neq 0$. The presence of the operators $m_{Y_{t}} \bar{Q}_{L} T_{R}$ and $m_{Y_{t}}^{\prime} \bar{Q}_{R} T_{L}$ modifies the potential but not its degree of divergence.

One can also understand this result from Feynman diagram considerations. For instance, the contribution to the quartic term in $\Phi$, at leading order in insertions of $m_{\psi}$ and $\Delta_{\psi}$ is given by:

(a)

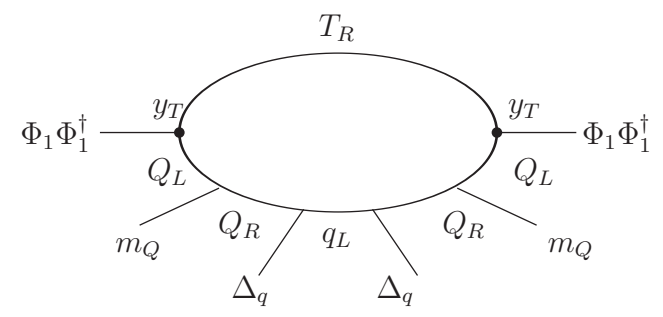

(b)

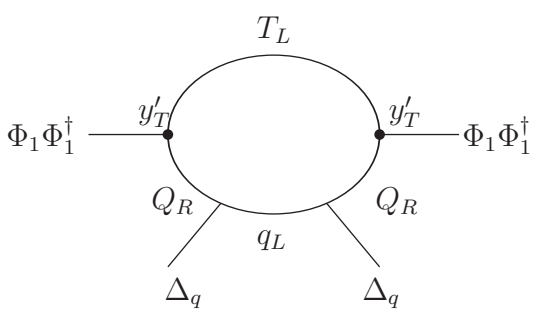

and similar diagrams changing $q \leftrightarrow t$ and $Q_{L, R} \leftrightarrow T_{R, L}$. These diagrams allow to understand the superficial degree of divergence of $V_{H}$ depending on which operators are present in the theory.

\section{Higgs phenomenology}

We turn now to the Higgs phenomenology of the composite Higgs models previously described. We present in this section the results of a detailed numerical analysis obtained by scanning over a sizeable region of the parameter space of each model. The minimization of the Higgs potential will be fully taken into account. Note, however, that we assume that for the light fermion generations both the LH and $\mathrm{RH}$ chiralities have a small degree of compositeness, as opposed to allowing one of them to have a large mixing angle with the composite sector, and the other a very suppressed one that accounts for the small SM fermion mass [43-46]. This assumption is more natural given the EW precision tests, which indicate that the light quarks and leptons are mostly elementary, although one could imagine exploring the second option. As a result, the Higgs potential is affected mainly by the top and bottom sectors, as well as by the gauge sector of the models. Nevertheless, when discussing the Higgs decays we will take into account some of the light fermions, most prominently the $\tau$ lepton, as discussed below. 


\subsection{Numerical scan}

The effective description of a composite Higgs described in the previous sections depends on a number of parameters. The gauge sector is described at the Lagrangian level by the two decay constants $\left\{f_{\Omega}, f_{\Omega_{X}}\right\}$ and gauge couplings $\left\{g_{\rho}, g_{X}\right\}$ associated with the $\mathrm{SO}(5)$ and $\mathrm{U}(1)_{X}$ (composite) factors, while in the elementary sector one has the two gauge couplings $g_{0}$ and $g_{0}^{\prime}$ [see eqs. (2.4) and (2.7)]. The latter are related to the SM gauge couplings as given in eq. (2.17), while it is convenient to parametrize the composite gauge couplings in terms of the elementary/composite mixing angles of the gauge sector: $t_{\theta}=g_{0} / g_{\rho}$ and $t_{\theta_{X}^{\prime}}=g_{0}^{\prime} / g_{X}$. However, for simplicity, in our scan we will fix $g_{X}$ by imposing the relation discussed after eq. (2.17), so that there is effectively a single gauge mixing angle $t_{\theta}$. The two decay constants can in turn be exchanged for the two mass scales $m_{\rho}$ and $m_{X}$ defined in eq. (2.13), but it is more convenient to scan over a subset of the physical masses after taking into account the elementary/composite mixing effects (before including EWSB effects). Thus, we choose to scan over $m_{\tilde{\rho}}=\sqrt{1+t_{\theta}^{2}} m_{\rho}=m_{\rho} / c_{\theta}$ [see discussion of the last paragraph of section 2.2], and we also choose the variable $m_{\tilde{X}}=m_{X} / c_{\theta}$. However, since we focus on a region of parameter space with $t_{\theta} \ll 1$, quantitatively there is not a large difference between $m_{\tilde{\rho}}$ and $m_{\rho}$ or $m_{\tilde{X}}$ and $m_{X}$.

The fermion sector depends on a set of "diagonal" masses $m_{\Psi}$, one for each composite fermion, and on the "off-diagonal" masses $m_{y_{u}}$ and $m_{y_{d}}$ of eq. (3.1). The composite sector also involves a number of "Yukawa-like" mass parameters that we have called $y_{u}$ and $y_{d}$ [see eqs. (3.9), (3.15), (3.21), (3.26), (3.36), (3.37) and (3.42) which define these for each model]. In spite of the notation, the $y_{\psi}$ have dimensions of mass, although they represent interactions with the Higgs field $\Phi$. Finally, there are the mixing parameters, $\Delta_{q}, \Delta_{u}$ and $\Delta_{d}$, which also have mass dimension 1 . In practice, the scan will be restricted to the third generation, so that one should reinterpret the indices as $u \rightarrow t$ and $d \rightarrow b$. We find convenient to exchange the mixing parameters $\Delta_{\psi}$ for "mixing angles" defined by $t_{\psi} \equiv \tan \theta_{\psi}=\Delta_{\psi} / m_{\Psi}$, where $\Psi$ is the composite fermion associated with the elementary fermion $\psi$ [for the $\mathrm{MCHM}_{5}$ we introduce two mixing angles $t_{q^{u}}$ and $t_{q^{d}}$ corresponding to $\Delta_{q^{u}}$ and $\Delta_{q^{d}}$; see comments after eq. (3.1)]. Analogously to the gauge sector above, we also prefer to scan over diagonal fermion masses that have been rescaled according to $m_{\tilde{\Psi}}=m_{\Psi} / c_{\psi}$, where $c_{\psi}=\cos \theta_{\psi}$ involves the corresponding mixing angle defined above. This choice leads to light custodians when the mixings are large, since their masses are given by $m_{\text {cust }} \sim \mathcal{O}\left(m_{\tilde{\Psi}} c_{\psi}\right)[47,48]$. Thus, the parameters for the fermionic sector consist of $\left\{m_{\tilde{\Psi}}, t_{\psi}, m_{y_{\psi}}, y_{\psi}\right\}$, where the indices run over the field content in each model, as described in section 3 [we fix $Z_{\psi}=1$ in eq. (3.1)].

Since one expects that the masses of the various resonances will be of the same order, for simplicity we have fixed a common mass scale, by restricting our scan to $m_{\tilde{\rho}}=m_{\tilde{Q}}=m_{\tilde{U}}=$ $m_{\tilde{D}}$ (for the $\mathrm{MCHM}_{5}$ we impose the condition on $m_{\tilde{Q}^{u}}$ and $m_{\tilde{Q}^{d}}$ ). This is not necessary, but we do not expect that the results will depend on this simplifying assumption. ${ }^{13}$ Thus,

\footnotetext{
${ }^{13}$ Note that the physical masses are obtained after taking into account all the mixing effects, as well as EWSB, and will therefore present a nontrivial spread. It is also worth noting that by scanning over $m_{\tilde{\rho}}$, $m_{\tilde{X}}$ and $m_{\tilde{\Psi}}$, i.e. by factoring out the elementary/composite mixing angles, we are proceeding in analogy
} 
the final set of parameters used in the scan is

$$
\left\{f_{h}, m_{\tilde{\rho}}, t_{\theta}, t_{q}, t_{t}, t_{b}, m_{y_{T}}, m_{y_{B}}, y_{T}, y_{B}\right\}
$$

where we used the notation $y_{T}$ and $y_{B}$ instead of $y_{t}$ and $y_{b}$ to avoid confusion with the SM top and bottom Yukawa couplings, and we also included in the list the Higgs decay constant $f_{h}$ defined by eqs. (2.3) and (2.9). We also chose to fix $m_{\tilde{X}}=s_{\theta_{W}} / \sqrt{c_{2 \theta_{W}}} m_{\tilde{\rho}} \approx 0.65 m_{\tilde{\rho}}$, which amounts to fixing $f_{\Omega_{X}}=f_{\Omega}$ in eq. (2.4), given the choice of $g_{X}$ described above. We choose $1 / 5 \leq t_{\theta} \leq 1 / 3$, so that $g_{\rho}$ is large but perturbative, and scan over the fermionic mixing angles according to $s_{\psi} \in[0.4,1]$, with a uniform distribution (but we adjust $s_{b}$ to reproduce the bottom quark mass with little effect on the EWSB properties of the parameter point). For the mass parameters, $\left(m_{\tilde{\rho}}, m_{y_{T}}, m_{y_{B}}, y_{T}\right.$ and $\left.y_{B}\right)$, we scan in units of $f_{h}$ as follows:

- $m_{\tilde{\rho}} / f_{h} \in[2.5,5]$, which is consistent with the underlying relation $m_{\rho} \sim g_{\rho} f_{h}$ with $g_{\rho}$ in the range of interest,

- $\left|y_{\psi} / f_{h}\right|<2 \pi$, which encodes the idea of having a perturbative proto-Yukawa coupling,

- and $\left|m_{y_{T}} / f_{h}\right|,\left|m_{y_{B}} / f_{h}\right| \lesssim 2 \pi$,

while $f_{h}$ is scanned over a wide range, but we choose only points with $\epsilon<0.5$, which corresponds to $f_{h} \gtrsim 500 \mathrm{GeV}$. The final set of points has $f_{h}$ as large as $\sim 2.5 \mathrm{TeV}$ (except for the $\mathrm{MCHM}_{5-10-10}$, which has some points with $f_{h}$ as large as $\sim 6 \mathrm{TeV}$ ). We also required in the final set of points that $m_{\tilde{\rho}}>2 \mathrm{TeV}$. This final set of numbers already assumes that we have normalized to $m_{Z}$ (see below).

Having chosen a given point in the parameter space described above, we minimize the 1-loop Higgs potential to select those points that do break the EW symmetry. For each such point, we can rescale all parameters with dimension of mass so as to reproduce $m_{Z}$, thereby normalizing to the EW scale. We further select those points where the Higgs mass matches the measured value of $\sim 125 \mathrm{GeV}$, and also select those points where the top and bottom quarks match the experimental observations. In practice, our final points have $m_{h} \in[120-130] \mathrm{GeV}, m_{t} \in[140-170] \mathrm{GeV}$ and $m_{b} \approx 2.7 \mathrm{GeV} .{ }^{14}$ We can then compute the couplings of the Higgs to the vector bosons and fermions (both the SM ones as well as the new resonances), which are then used as input to compute the Higgs production cross sections and branching fractions. This is done numerically without any approximations, as

to the extra-dimensional realizations, where the compactification scale and therefore the overall KaluzaKlein (KK) scale is treated as an input parameter. The elementary/composite mixing angles of the 4D realization are related to the $5 \mathrm{D}$ localization parameters and boundary conditions for the various fields. When obtaining the exact spectrum one can get modes much lighter than the overall KK scale, typically for large mixing angles in the third generation fermionic sector.

${ }^{14}$ We note that the relevant masses from the point of view of the scan should be the running masses at the scale where the heavy resonances are integrated out. These would then be run down to the weak scale with the SM RGE's to make contact with the experimental measurements. Since each parameter point has a different scale for the heavy resonances, we have simply defined generous windows to capture the spirit of the matching procedure. Although a more precise analysis is possible, we do not expect that the conclusions will change. 

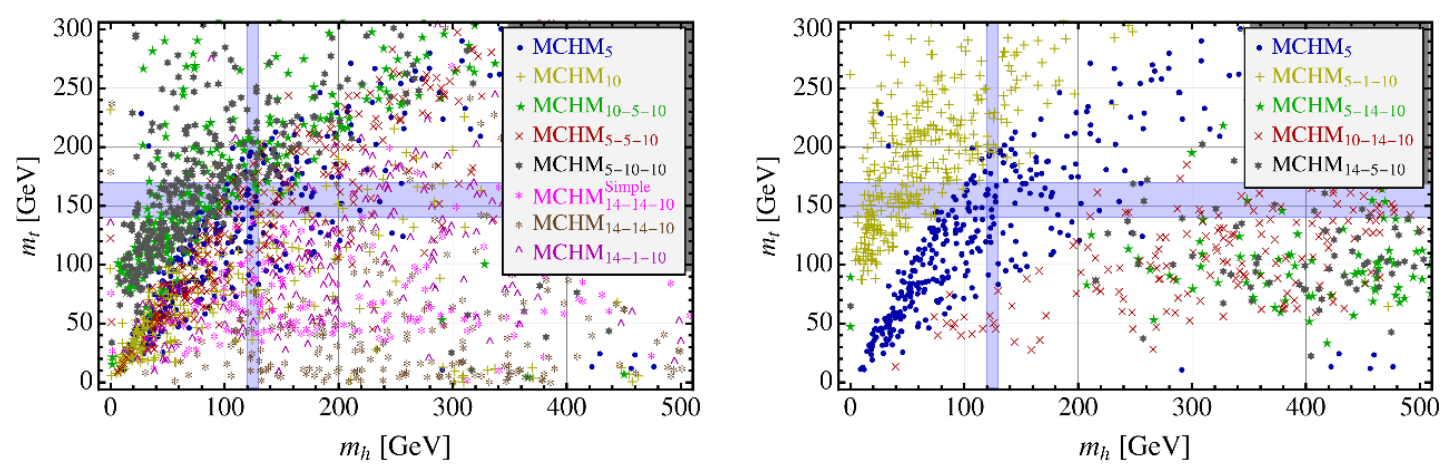

Figure 2. A random subset of the points that present electroweak symmetry breaking, but without requirements on the Higgs, top or bottom masses (however, we have normalized to $m_{Z}$ ). The vertical and horizontal bands indicate the windows we have defined for $m_{h}$ and $m_{t}$. In the left panel we show the models we have presented in detail in section 3. In the right panel we show the models mentioned in section 3.8, showing again the $\mathrm{MCHM}_{5}$ for comparison.

is done for the 1-loop induced couplings ( $h g g, h \gamma \gamma$ and $h Z \gamma)$ which are computed using the exact spectrum and couplings to the Higgs. However, we also compare to the analytical approximation described in section 4, which in general gives a qualitative understanding of the numerical scan.

In figure 2 we display a random subset of the scanned points that display EWSB, in the plane of $m_{t}$ versus $m_{h}$ (after normalization to $m_{Z}$ ). We have not imposed here any requirements on $m_{h}, m_{t}$ nor $m_{b}$, only that the desired symmetry breaking pattern be obtained and that the $b_{R}$ mixing angle be suppressed (as is necessary to obtain a light bottom quark in models with just one operator coupled to $q_{L}$ ). In the left panel we present the (color coded) models described in detail in section 3, showing that some of the models reproduce more naturally the Higgs and top masses than others. In particular, the models involving the 14 representation have a tendency to produce a too large $m_{h}$ [33], although one can find a few points in the desired range at the price of tuning (the bands correspond to the windows we have defined in the previous paragraph).

In the right panel, we show the same information for the models mentioned without details in section 3.8, together with the $\mathrm{MCHM}_{5}$ for comparison purposes. We see that these models also typically do not fall in the phenomenologically desired window: for the $\mathrm{MCHM}_{5-1-10}$ the quartic coupling is usually too small, since the only source of breaking is the mixing with $q_{L}$, that leads to a factor $s_{h}$ in $\Pi_{u_{L}}$ and $s_{h}^{2}$ in $M_{u}$, in agreement with the results found in [33]. The $\mathrm{MCHM}_{10-14-10}$ leads to a heavy Higgs. The $\mathrm{MCHM}_{14-5-10}$ and $\mathrm{MCHM}_{5-14-10}$ allow for two independent proto-Yukawa interactions: $\mathcal{L}_{y} \supset y_{u} \bar{\Psi}_{5} \Psi_{14} \Phi+$ $\tilde{y}_{u}\left(\bar{\Psi}_{5} \Phi\right)\left(\Phi^{\dagger} \Psi_{14} \Phi\right)$, similar to the $\mathrm{MCHM}_{14-14-10}$. Both of them generically lead to a heavy Higgs, while EWSB prefers $\tilde{y}_{u} \neq 0$ for the $\mathrm{MCHM}_{14-5-10}$ and $y_{u} \neq 0$ as well as $\tilde{y}_{u} \neq 0$ for the $\mathrm{MCHM}_{5-14-10}$. For the remaining three models we did not find points with the proper $m_{h}$ and $m_{t}$ by performing a random scan. Finally, the $\mathrm{MCHM}_{14-10-10}$ generically does not lead to EWSB.

In all these models there is a correlation between $m_{h}$ and $m_{t}$ [55], that can usually be approximated by: $m_{h}^{2} \sim a \frac{N_{c}}{\pi^{2}} \frac{m_{t}^{2}}{f_{h}^{2}} m_{\psi}^{2}$, with $m_{\psi}$ the scale of the lightest fermionic resonance 

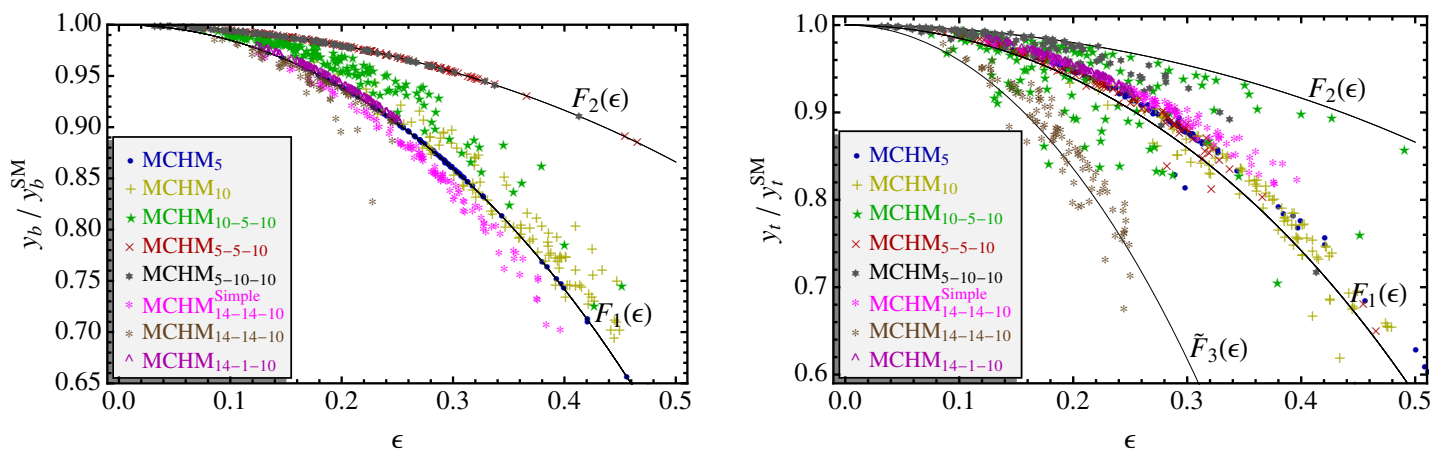

Figure 3. Bottom (left panel) and top (right panel) Yukawa couplings in several models, normalized to the SM (defined as $y_{\psi}^{\mathrm{SM}}=m_{\psi} / v_{\mathrm{SM}}$ with $v_{\mathrm{SM}}=246 \mathrm{GeV}$ ). The points correspond to a random scan in parameter space, while the solid curves correspond to the analytic approximation discussed in the main text.

cutting off the 1-loop potential and $a$ a factor that is model dependent. Usually $a \sim \mathcal{O}(1)$, however in some cases it can be suppressed $a \sim \mathcal{O}\left(\epsilon^{2}\right)$ or enhanced $a \sim \mathcal{O}\left(\epsilon^{-2}\right)$, as shown in [33]. The analytical approximations of [33] are in qualitative agreement with the full numerical results of figure 2.

From here on we focus on the models described in detail in section 3, which seem to be phenomenologically preferred due to the previous observations. As mentioned earlier, we analyze the $\mathrm{MCHM}_{14-14-10}$ in detail, even though it tends to produce too heavy a Higgs, as it may serve also to illustrate the situation in those models we do not elaborate any further. All the numerical results of the following sections correspond to points that lie at the intersection of two bands of figure 2 .

\subsection{Corrections to the gauge and Yukawa couplings}

We start by comparing the simple analytical approximation described in section 4 for the deviations in the Higgs couplings to the SM gauge bosons and fermions w.r.t. the SM expectation [see also the discussion after eq. (3.6)]. As discussed there, this approximation is expected to work well when the elementary/composite mixing angles are small, which typically happens for the light fermions in our scenario. However, we find that even for the top quark, the approximation $y_{t} \approx\left[F_{t}(\epsilon) /\left(\epsilon f_{h}\right)\right] m_{t}$ is reasonably good, even when the mixing angles are sizeable, provided there are no "ultra-light" fermionic resonances. This is illustrated in figure 3, where we show the bottom and top Yukawa couplings as a function of $\epsilon$ in several models (normalized to the corresponding SM Yukawa coupling, $y_{\psi}^{\mathrm{SM}} \equiv m_{\psi} / v_{\mathrm{SM}}$ with $\left.v_{\mathrm{SM}} \approx 246 \mathrm{GeV}\right)$. The points correspond to a random scan over the parameter space described in the previous subsection, while the solid curves correspond to the approximation described in section 4 (see table 1 ).

We see in the left panel of figure 3 that the approximation described in eq. (4.2) works very well for the bottom sector all the way up to relatively large values of $\epsilon$. A notable exception occurs for the $\mathrm{MCHM}_{10-5-10}$ (green stars), where the analytic expectation, $F_{1}=$ $\cos \left(2 v / f_{h}\right) / \cos \left(v / f_{h}\right)$, systematically overestimates the suppression in $y_{b}$ compared to the SM. The sizeable deviation observed can be understood by considering the next to leading 

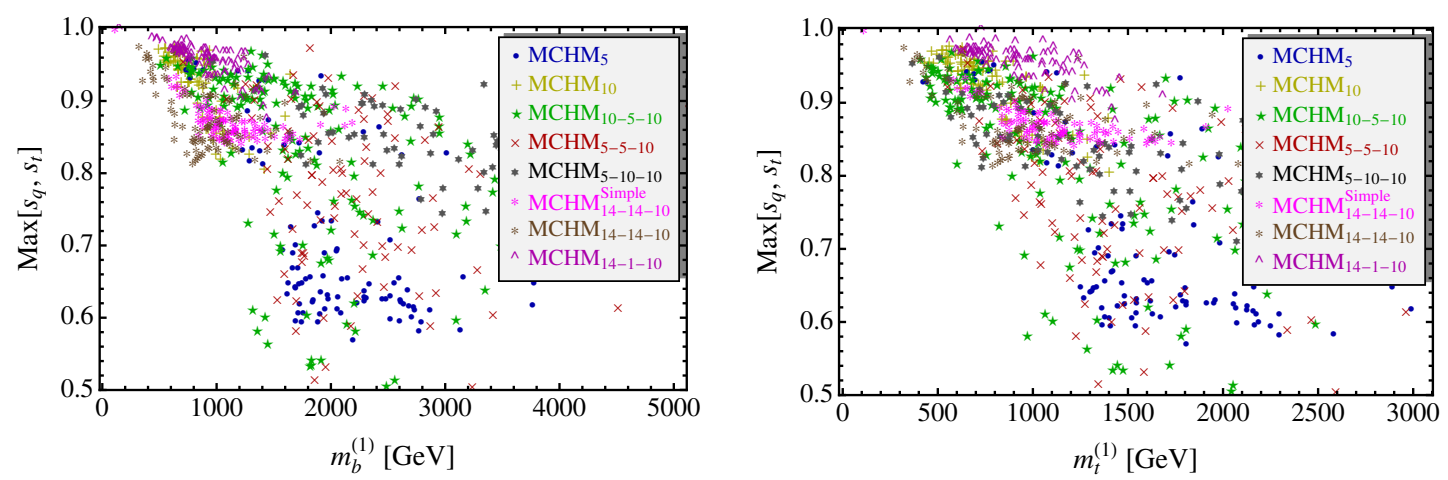

Figure 4. The largest of the mixing angles between $s_{q}$ and $s_{t}$ versus the lightest $Q=-1 / 3$ resonance (left panel) and $Q=2 / 3$ resonance (right panel) in several models. For the $\mathrm{MCHM}_{5}$ we plot the largest between $s_{q_{u}}, s_{q_{d}}$ and $s_{t}$.

order term in the expansion of $y_{b} / y_{b}^{S M}$ in powers of $\epsilon$, as shown in section 3. We obtain that, after the selection of points explained above, the coefficient of the $\mathcal{O}\left(\epsilon^{2}\right)$ term for the $\mathrm{MCHM}_{10-5-10}$ is of $\mathcal{O}(0.5)$. In contrast, the corresponding coefficient for the $\mathrm{MCHM}_{10}$, $\mathrm{MCHM}_{14-14-10}$ and $\mathrm{MCHM}_{14-14-10}^{\text {simple }}$ is of $\mathcal{O}(0.1),{ }^{15}$ for the $\mathrm{MCHM}_{5}$ and $\mathrm{MCHM}_{14-1-10}$ it is of $\mathcal{O}\left(10^{-2}\right)$, and for the $\mathrm{MCHM}_{5-5-10}$ and $\mathrm{MCHM}_{5-10-10}$ it is $\mathcal{O}\left(10^{-4}\right)$, in all the cases increasing with $s_{q}$ as expected. Since $h \rightarrow b \bar{b}$ is the dominant decay mode, deviations of $y_{b}$ can have a deep impact in the Higgs phenomenology.

It is also interesting to note that the bulk of the points in the $\mathrm{MCHM}_{10-5-10}$ display relatively light $(Q=-1 / 3)$ fermionic resonances, together with relatively large mixing angles. We illustrate this in the left panel of figure 4, where we show the largest of the mixing angles $\left(s_{q}, s_{t}\right)$ versus the lightest vectorlike resonance mass in the bottom sector. Indeed, most of the green stars $\left(\mathrm{MCHM}_{10-5-10}\right)$ exhibit resonances below $1 \mathrm{TeV}$ and $s_{q}>0.9$. Note that the $\mathrm{MCHM}_{10}$ (yellow +'s), the $\mathrm{MCHM}_{14-14-10}$ (brown *'s), and to a somewhat lesser extent the $\mathrm{MCHM}_{14-14-10}^{\text {simple }}$ (magenta *'s), also contain a subset of points with light states together with sizeable elementary-composite mixing angles, which is reflected in the somewhat larger dispersion in figure 3, compared to the other models. However, note that the $\mathrm{MCHM}_{14-1-10}$ (dark magenta ' 's) has light $Q=-1 / 3$ resonances together with large mixing angles, and nevertheless follows the naive approximation from eq. (4.2) for the bottom Yukawa coupling rather well.

The right panels of figures 3 and 4 display the same information for the top sector (using the lightest $Q=2 / 3$ fermionic resonance as the relevant variable). Here, the dispersion of the points around the continuous curves is larger, but the general behavior is still well described by the simple analytic formulas given above, again with the exception of the $\mathrm{MCHM}_{10-5-10}$ (green stars), which all fall below the "expected curve" given by $F_{2}=\cos \left(v / f_{h}\right)$. Thus, the analytic approximation underestimates the suppression in the top Yukawa coupling compared to the SM in this model. We also note here that the analytic approximation, $F_{1}(\epsilon)$, slightly underestimates the exact result for the $\mathrm{MCHM}_{10}$,

\footnotetext{
${ }^{15} \mathrm{MCHM}_{14-14-10}^{\text {simple }}$ refers to the model described in section 3.6 with $\tilde{y}_{T}=0$ in eq. (3.6) [making $u \rightarrow T$ ]. We refer to the general model with $y_{T}, y_{B}$ and $\tilde{y}_{T}$ turned on as $\mathrm{MCHM}_{14-14-10}$.
} 
$\mathrm{MCHM}_{14-14-10}$ and the $\mathrm{MCHM}_{14-1-10}$ (with the effect being more pronounced for the latter two). Finally, we point out that after imposing the physical conditions described in the previous section, the points in the $\mathrm{MCHM}_{14-14-10}$ typically have $y_{T} \ll \tilde{y}_{T}$. This means that the deviations from the SM in the top sector are reasonably well described by the function $\tilde{F}_{3}(\epsilon)$ [see discussion around eq. (4.5)], as can be seen in the right panel of figure 3 .

Besides the above resonances, one can also find light exotic resonances with charge $Q=8 / 3,5 / 3$ and $-4 / 3$, depending on the fermion representations involved. These resonances are also custodians, thus their masses are also suppressed if they belong to $\mathrm{SO}(5)$ multiplets with large mixing with the elementary fermions. They can have a rich and exciting phenomenology at colliders, although we will not consider this issue in this work.

The Yukawa couplings of the light fermions should be very well described by the analytical approximations, at least when both LH and RH mixing angles are small, as we are assuming. In particular, all of them can be expected to deviate from the SM expectation by the same order as the couplings of the third generation, reflecting the "universal" character of the leading order deviations found in composite Higgs scenarios (those parametrized by the $F_{i}$ functions of table 1 ).

\subsection{Higgs production and decay}

Based on the above observations, we can write simple analytical expressions for the Higgs branching fractions and production rates that allow us to understand the qualitative (and often quantitative) behavior. However, for the numerical computations in the scan we will not perform any such approximations, as already mentioned.

For the tree-level Higgs decays, we have

$$
\begin{aligned}
\Gamma(h \rightarrow b \bar{b}, \tau \tau) & \approx \Gamma_{\mathrm{SM}}(h \rightarrow b \bar{b}, \tau \tau) \times r_{b}^{2}(\epsilon), \\
\Gamma(h \rightarrow c \bar{c}) & \approx \Gamma_{\mathrm{SM}}(h \rightarrow c \bar{c}) \times r_{c}^{2}(\epsilon), \\
\Gamma(h \rightarrow W W, Z Z) & \approx \Gamma_{\mathrm{SM}}(h \rightarrow W W, Z Z) \times r_{V}^{2}(\epsilon),
\end{aligned}
$$

where $\Gamma_{\mathrm{SM}}(h \rightarrow i)$ is the SM Higgs partial decay width in the $i$-th channel. We have assumed here that the leptons (in particular the $\tau$ ) are in the same $\mathrm{SO}(5)$ representations as the bottom quark. Similarly, all up-type quarks (in particular, charm and top) will be assumed to belong to the same $\mathrm{SO}(5)$ representation, hence $r_{c}(\epsilon)=r_{t}(\epsilon)$, which can be read from table. 1 for the different models. ${ }^{16}$

For the loop-level Higgs decays, we write

$$
\begin{aligned}
& \frac{\Gamma(h \rightarrow g g)}{\Gamma_{\mathrm{SM}}(h \rightarrow g g)} \approx \frac{\left|r_{t}(\epsilon) A_{1 / 2}\left(m_{h}^{2} / 4 m_{t}^{2}\right)+r_{b}(\epsilon) A_{1 / 2}\left(m_{h}^{2} / 4 m_{b}^{2}\right)\right|^{2}}{\left|A_{1 / 2}\left(m_{h}^{2} / 4 m_{t}^{2}\right)+A_{1 / 2}\left(m_{h}^{2} / 4 m_{b}^{2}\right)\right|^{2}} \\
& \frac{\Gamma(h \rightarrow \gamma \gamma)}{\Gamma_{\mathrm{SM}}(h \rightarrow \gamma \gamma)} \approx \frac{\left|r_{V}(\epsilon) A_{1}\left(\frac{m_{h}^{2}}{4 m_{W}^{2}}\right)+N_{c} Q_{t}^{2} r_{t}(\epsilon) A_{1 / 2}\left(\frac{m_{h}^{2}}{4 m_{t}^{2}}\right)+N_{c} Q_{b}^{2} r_{b}(\epsilon) A_{1 / 2}\left(\frac{m_{h}^{2}}{4 m_{b}^{2}}\right)\right|^{2}}{\left|A_{1}\left(m_{h}^{2} / 4 m_{W}^{2}\right)+N_{c} Q_{t}^{2} A_{1 / 2}\left(m_{h}^{2} / 4 m_{t}^{2}\right)+N_{c} Q_{b}^{2} A_{1 / 2}\left(m_{h}^{2} / 4 m_{b}^{2}\right)\right|^{2}}
\end{aligned}
$$

\footnotetext{
${ }^{16}$ If different generations are assigned to different $\mathrm{SO}(5)$ representations it is straightforward to generalize our expressions by simply computing the corresponding $F_{\psi}(\epsilon)$ from eq. (4.1), although it may happen that this function has additional dependence on other microscopic parameters.
} 

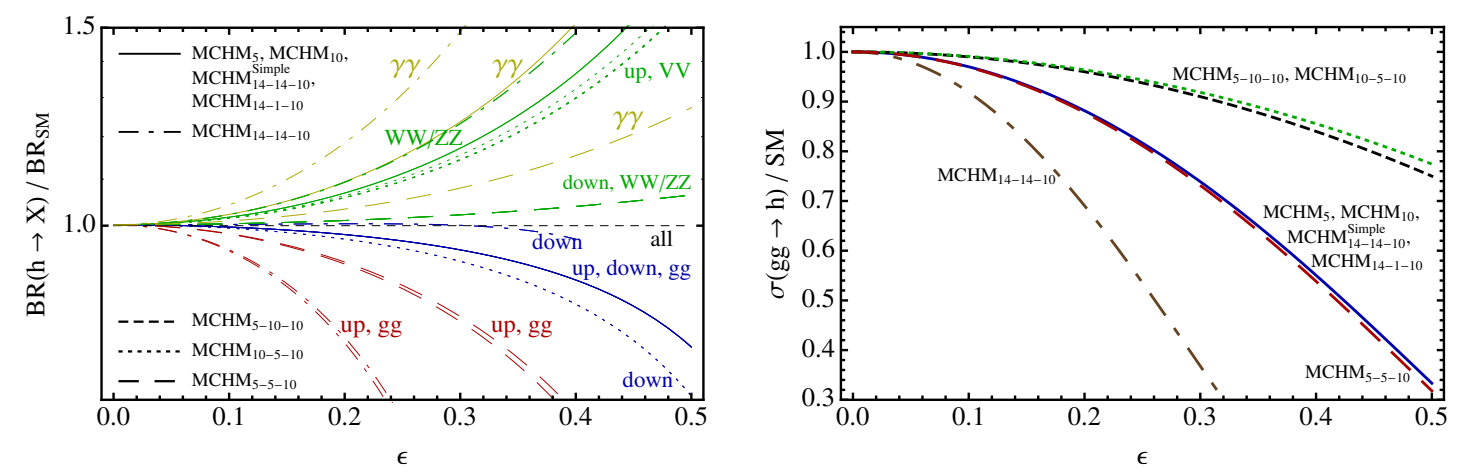

Figure 5. Left panel: branching fractions (normalized to the SM) into fermions and gauge bosons for several models following from the approximation in eq. (4.2). Here $V V=W W, Z Z, \gamma \gamma, g g$. The color coding of the lines matches the color coding of the closest legend. Right panel: gluon fusion production cross section (normalized to the SM) in those models. The vector boson fusion (VBF) cross section coincides with the curve marked as " $\mathrm{MCHM}_{5-10-10}, \mathrm{MCHM}_{10-5-10}$ ".

where $A_{1 / 2}(\tau)$ and $A_{1}(\tau)$ are the well-known loop functions (see appendix D), $N_{c}=3$ is the number of colors and $Q_{t}=2 / 3, Q_{b}=-1 / 3$ are the top and bottom quark electric charges, respectively. Note that here we have formally included only the effects of the zeromodes, since in the limit where eq. (4.2) holds, the contribution of the associated towers of heavy resonances becomes negligible. However, to the extent that $A_{1 / 2}\left(\frac{m_{h}^{2}}{4 m_{t}^{2}}\right) \approx 4 / 3$ (its asymptotic value for $4 m_{t}^{2} \gg m_{h}^{2}$ ), and given the sum rule eq. (4.1), the above set of approximations effectively include the effects of the full top tower. For the bottom quark contribution, the situation is different since $\left|A_{1 / 2}\left(m_{h}^{2} / 4 m_{b}^{2}\right)\right| \approx 1 / 16 \ll 1$ for $m_{h} \approx 125 \mathrm{GeV}$ and $m_{b} \approx 2.7 \mathrm{GeV}$. In addition, in some cases (as in the $\mathrm{MCHM}_{10-5-10}$ ), the contribution of the heavy towers can be as large as $10 \%$ of the sum in eq. (4.1). As a result, the contribution of the heavy $Q=-1 / 3$ states to the above loop-induced processes can be of the same order as the actual contribution of the bottom quark, since although $y_{b} / m_{b}$ still dominates the sum in eq. (4.1), it has to be multiplied by the small $A_{1 / 2}\left(m_{h}^{2} / 4 m_{b}^{2}\right)$ for the physical processes. Given that the contribution of the bottom-like resonances is not included in eqs. (6.5) and (6.6), our approximation could carry an uncertainty of the same order as the bottom contribution, which can be as large as $10 \%$. However, for most models, the approximation is significantly better.

In the left panel of figure 5, we show the Higgs branching fractions into fermion and gauge boson pairs in the $\mathrm{MCHM}_{5}, \mathrm{MCHM}_{10}, \mathrm{MCHM}_{14-14-10}^{\text {Simple }}, \mathrm{MCHM}_{14-1-10}$ (solid lines), $\mathrm{MCHM}_{14-14-10}$ (dash-dotted lines), $\mathrm{MCHM}_{5-10-10}$ (short dashed lines), $\mathrm{MCHM}_{10-5-10}$ (dotted lines), and $\mathrm{MCHM}_{5-5-10}$ (long dashed lines). We see that in some cases the BR's are enhanced with respect to the SM while in others they are suppressed. One should notice that all partial decay widths always present a suppression, in particular for the $b \bar{b}$ decay channel. As a result the total decay width is suppressed, and the BR's in some channels can end up being enhanced due to the smaller denominator. In contrast, the Higgs production cross sections are always suppressed with respect to the SM, as shown in the right panel of figure 5 for the gluon fusion Higgs production cross section, normalized 

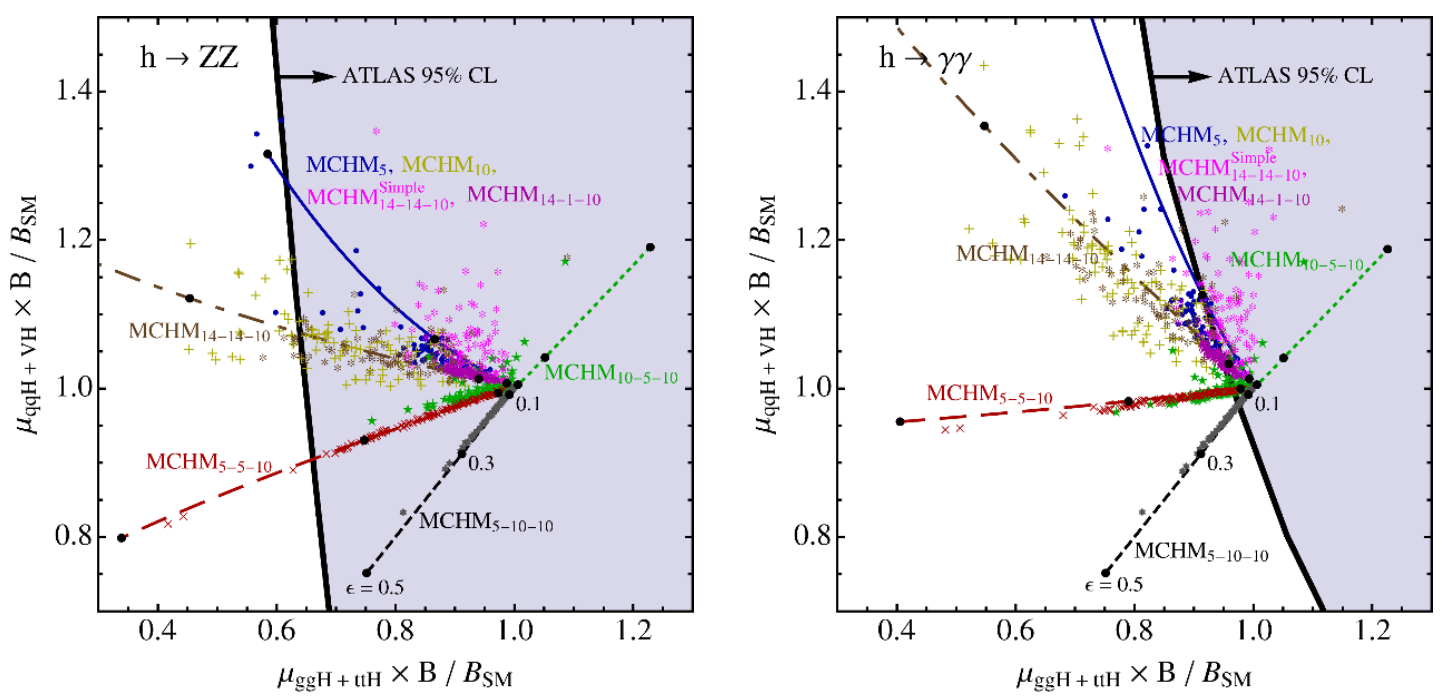

Figure 6. Left panel: rates in the $h \rightarrow Z Z$ decay channel separated according to production mode: gluon fusion $(+t \bar{t} h)$ versus $\operatorname{VBF}(+h W / Z)$. The larger black dots indicate the positions of $\epsilon=0.1,0.3,0.5$. Right panel: same for the $h \rightarrow \gamma \gamma$ channel. The solid curves correspond to the analytical approximation discussed in the main text, while the points correspond to a random scan that reproduces $m_{h} \sim 125 \mathrm{GeV}, m_{t} \sim 160 \mathrm{GeV}$ and $m_{b} \sim 4 \mathrm{GeV}$. The shaded region corresponds to the current 95\% CL curve by ATLAS. The CMS 95\% CL region would cover the full area of the figure. The production signal strengths are defined as $\mu_{i}=\sigma^{\operatorname{Model}}(i) / \sigma^{\mathrm{SM}}(i)$. The production cross sections used correspond to the $8 \mathrm{TeV}$ run of the LHC.

to the SM. We also note that the VBF production cross section coincides with the upper curve in this plot.

Consequently, the total cross sections in given channels can be enhanced or suppressed with respect to the $\mathrm{SM}$, depending on how these opposing effects play out. We illustrate this in figure 6 for the $Z Z$ (left panel) and $\gamma \gamma$ (right panel) decay modes, separating the gluon fusion $(+t \bar{t} h)$ production from $\operatorname{VBF}(+h W / Z)$, as done by the ATLAS and CMS collaborations [49]. The continuous lines correspond to the expectation based on the above analytical approximation. We have superimposed the exact predictions for the scan in the models we consider. We see that the approximation tracks well the actual analytical predictions for all models (up to some dispersion due to the effect of the bottom sector explained above), except for the $\mathrm{MCHM}_{10-5-10}$ on which we comment further below. One can understand the behavior of these curves from figure 5. For instance, for the $\mathrm{MCHM}_{5-10-10}$, since all channels (gauge, down-type and up-type) are suppressed by exactly the same $r(\epsilon)$, the BR's remain exactly as in the SM, while the production in all modes is suppressed identically. Thus, the curve points at a $45^{\circ}$ angle towards the left-down, as $\epsilon=\sin (v / f)$ increases and the deviations from the SM increase. The $\mathrm{MCHM}_{5-5-10}$ shows a very mild enhancement in the $Z Z$ and $\gamma \gamma$ BR's (see left panel of figure 5), which is not enough to compensate the suppression in production. Since the latter is more significant in gluon fusion than in VBF, the curve in figure 6 points to the left-down but closer to the horizontal than for the $\mathrm{MCHM}_{5-10-10}$. For the $\mathrm{MCHM}_{5}, \mathrm{MCHM}_{10}, \mathrm{MCHM}_{14-14-10}$, $\mathrm{MCHM}_{14-14-10}^{\text {Simple }}$ and $\mathrm{MCHM}_{14-1-10}$, the left panel of figure 5 shows a stronger enhancement 
in both $\mathrm{BR}(h \rightarrow Z Z)$ and $\mathrm{BR}(h \rightarrow \gamma \gamma)$, which is sufficient to compensate the suppression in the VBF production but not enough to compensate the significant suppression in gluon fusion (see right panel of figure 5). As a result, the analytical prediction curves to the left-up. Note, however, that the scanned points for the $\mathrm{MCHM}_{14-14-10}^{\text {Simple }}$ show a more pronounced tendency to compensate the suppression in gluon fusion by the enhancement in the branching fractions than the naive analytical expectation. This can be traced to the systematic (albeit small) deviations exhibited in figure 3 for the top and bottom Yukawa couplings. Finally, we see that the analytical prediction for the $\mathrm{MCHM}_{10-5-10}$ does not reproduce the qualitative behavior of the scan. While a line at $45^{\circ}$ to the right-up is expected (from figure 5 one can see that the enhancement in BR's dominates over the suppression in production in all the modes), most of the points actually present a suppression with respect to the SM. This can be traced back to our previous comments in regards to this model: the analytical approximation systematically overestimates the suppression in the $b \bar{b}$ channel [hence overestimates the enhancement in $\operatorname{BR}(h \rightarrow Z Z)$ and $\operatorname{BR}(h \rightarrow \gamma \gamma)$ ], while it systematically underestimates the suppression in the top Yukawa coupling, which translates into an overestimate of the gluon fusion Higgs production rate. These $\mathcal{O}(10 \%)$ errors are sufficient within this model to change the qualitative behavior. The VBF production is still well described by the analytic approximation, as is for all the other models, since the gauge resonances are always heavy.

It is interesting that the different fermionic representations lead to a different behavior in the plane of figure 6 , so that a precise measurement of these rates could be used to distinguish between different scenarios (although there could still remain a degeneracy between the $\mathrm{MCHM}_{5}, \mathrm{MCHM}_{10}, \mathrm{MCHM}_{14-14-10}^{\text {Simple }}$ and $\mathrm{MCHM}_{14-1-10}$, which in fact could be confused with the more general $\mathrm{MCHM}_{14-14-10}$ ). We also show the current $95 \%$ C.L. ellipse from the ATLAS analysis [49], and indicate the position along the solid line in each model that corresponds to $\epsilon=0.1,0.3,0.5$. We see that the experimental uncertainties still allow for relatively large values of $\epsilon$. The $95 \%$ C.L. ellipse from the CMS analysis would fill the region shown, so we do not indicate it.

The ATLAS and CMS collaborations have measured other properties of the $125 \mathrm{GeV}$ resonance. For instance, by taking channel by channel ratios of the $g g H+t t H$ and $q q H+V H$ production modes, and performing a fit to the data, they can set a bound on $\mu_{q q H+V H} / \mu_{g g H+t t H}$. This analysis only assumes that the same boson $H$ is responsible for all observed Higgs-like signals and that the separation of gluon-fusion like events and VBF-like events, based on the event kinematics, is valid. For instance, the ATLAS collaboration sets a bound of $\mu_{q q H+V H} / \mu_{g g H+t t H}=1.2_{-0.5}^{+0.7}$ [50]. The models in our scan have $1 \lesssim \mu_{q q H+V H} / \mu_{g g H+t t H} \lesssim 1.5$, so that they are not yet probed by these analyses. However, if a ratio below one was established it would disfavor the pNGB Higgs scenarios based on the lowest dimensional representation of $\mathrm{SO}(5)$. This is a manifestation of the generally important suppression in the gluon fusion process w.r.t. the SM. ATLAS also sets bounds on the Higgs production by gluon fusion alone, in terms of the rescaling factor $\kappa_{g}$. However, the analysis assumes that all the BR's are as in the SM and therefore does not apply to the present case.

From the LHC data one can also derive bounds on ratios of branching ratios, e.g. on $\rho_{\gamma \gamma / Z Z}=\left[\operatorname{BR}(\gamma \gamma) / \operatorname{BR}(\gamma \gamma)_{\mathrm{SM}}\right] /\left[\operatorname{BR}(Z Z) / \mathrm{BR}(Z Z)_{\mathrm{SM}}\right]$, etc. ATLAS finds $\rho_{\gamma \gamma / Z Z}=$ 

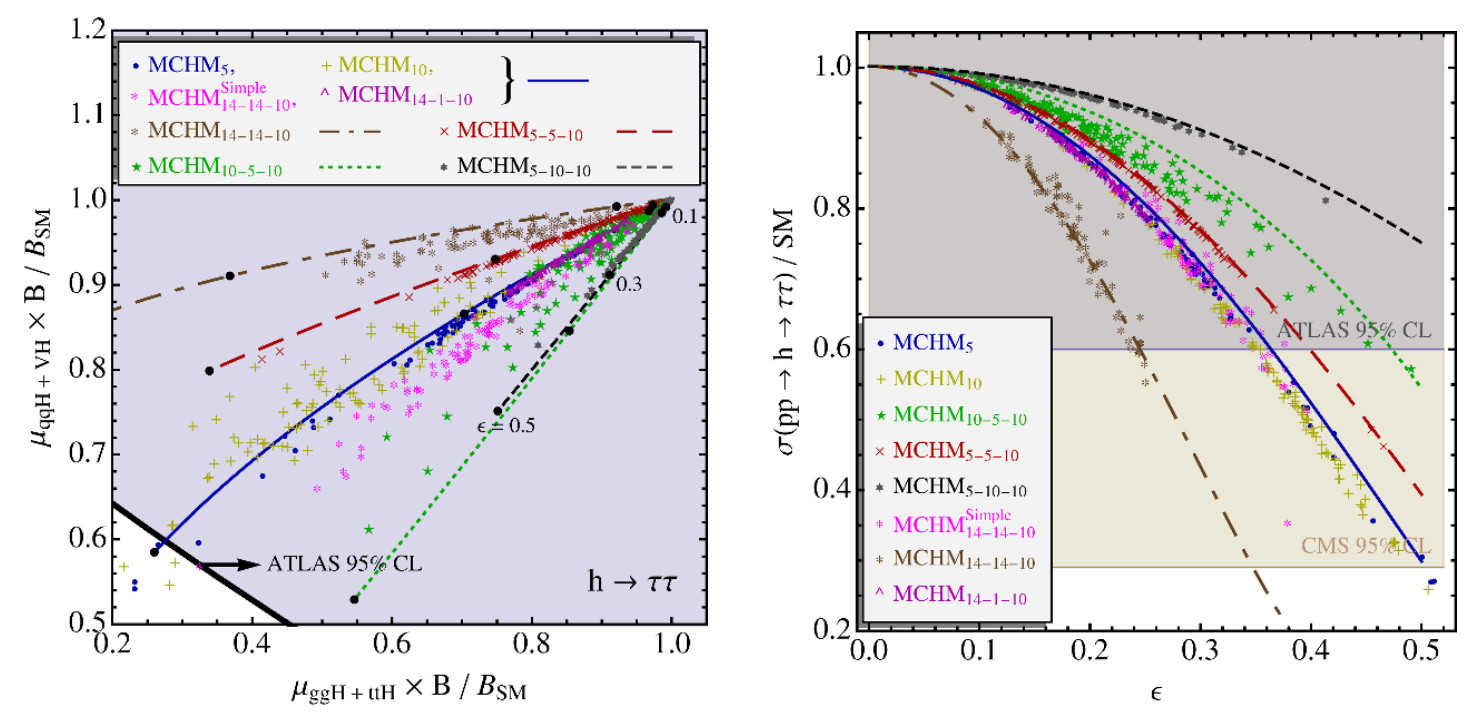

Figure 7. Left panel: similar to figure 6 , but for the $h \rightarrow \tau \tau$ channel. Right panel: we show the total rate (i.e. inclusive production) in the $\tau \tau$ channel, normalized to the SM, versus $\epsilon$. The horizontal bands correspond to the 95\% C.L. limit set by the ATLAS [51] and CMS [52] collaborations.

$1.1_{-0.3}^{+0.4}[50]$. Our scans have $1 \lesssim \rho_{\gamma \gamma / Z Z} \lesssim 1.1$, so that they are not yet probed in such measurements. Similarly, due to the custodial symmetry, we have $\rho_{W W / Z Z} \approx 1$, and it would be very challenging to differentiate it from the SM at the LHC; a significant deviation from the custodial limit would disfavor both the SM and the pNGB scenarios we have studied.

Apart from the indirect sensitivity to the top quark via the loop processes above, the fermionic channels, in particular $h \rightarrow \tau \tau$ are starting to be measured with interesting precision $[51,52]$ for the present work, although the uncertainties are still sufficiently large to be consistent with the great majority of our parameter point sample. In the left panel of figure 7 we show the expectations for this channel, discriminating between the Higgs production by gluon fusion $(+t t H)$ and $\mathrm{VBF}(+V H)$, together with the $95 \%$ C.L. region from ATLAS. In the right panel we show the signal strength for the inclusive $h \rightarrow \tau \tau$ production as a function of $\epsilon$. The horizontal bands correspond to the $95 \%$ C.L. regions from ATLAS [51] and CMS [52]. We note that under our assumptions, the $\tau \tau$ channel is always suppressed w.r.t. the SM. However, one should remember that one may be able to consider different representations for the $\tau$ sector, without affecting the properties of the Higgs potential. Hence, establishing an enhancement in the $\tau \tau$ channel over the SM would be in conflict with our assumptions, but we cannot claim that it would rule out the general framework.

In contrast, in models with a minimal content of composite fermion multiplets, one expects a robust suppression w.r.t. the SM in the $h \rightarrow b \bar{b}$ decay mode, so that this would be an interesting channel to probe the scenario. We find a suppression of $10-20 \%$ for $\epsilon=0.3$ and $20-40 \%$ for $\epsilon=0.5$, with smaller dispersion between different models than in the $\tau \tau$ channel. This is because at the LHC one must consider $p p \rightarrow h+X \rightarrow b \bar{b}+X$ in order to be able to discriminate against the large QCD background, so that only $V B F+V H+$ $t t H$ contribute, but not $g g H$ which is most sensitive to the new fermionic resonances that 
distinguish between different models. Unfortunately, at the LHC the precision may not be sufficient to provide a clear test, but its high luminosity phase or a linear collider could set useful bounds.

\section{$6.4 h \rightarrow Z \gamma$}

We turn now to the last decay channel we consider: $h \rightarrow Z \gamma$, which has not yet been observed, but could be seen in the near future. The decay of a pNGB Higgs to $Z \gamma$ has received considerable attention recently. Ref. [37] has shown that there can be large corrections to this decay, while being simultaneously compatible with precision EW measurements, thus providing a very interesting test. In order to obtain a large effect in this decay the composite sector itself must break the $P_{L R}$ symmetry, otherwise the only source of $P_{L R}$ breaking is the interaction between the elementary and composite fields, and the effect is suppressed [40]. We have not considered breaking of $P_{L R}$ by the composite sector in our work, so that we expect small corrections in the $h \rightarrow Z \gamma$ channel. We have computed the corrections to this rate in the models presented in the previous sections. Below we discuss the main features of this decay and show our results.

In the SM the interaction $h Z_{\gamma}$ is a radiative effect, generated at 1-loop by virtual $W$ 's and fermions. Similar to $h \gamma \gamma$, the bosonic and fermionic contributions have opposite sign. The first one dominates over the second one by a factor $\sim 10$, and the fermionic loop is dominated by the top contribution. In the MCHM one can distinguish the corrections from the new particles in the loop from those arising from the modified couplings between the Higgs and the SM gauge and fermion fields, as was the case for the $h \gamma \gamma$ process. However, unlike in the $h \rightarrow \gamma \gamma$ diagrams, there can be two different particle species running in the loop, since only one of the external particles is a gauge field of an unbroken symmetry. Therefore, in theories with extra $W$ 's, besides the loop with a single heavy field there are 1-loop effects involving two different virtual states. We will refer to these contributions as "diagonal" and "non-diagonal", respectively. Similarly, in theories with new fermions there are 1-loop effects involving a single new fermion as well as effects involving propagators of two different fermion species. We will clarify below which diagrams give the leading contributions.

As in the SM, in the models we are considering there are no tree-level contributions to the $h \rightarrow Z \gamma$ process, so we focus on the 1-loop effects, starting with those due to bosonic fields. Each diagonal contribution is suppressed by a factor $\left(m_{W} / m_{W_{n}}\right)^{2} \sim \mathcal{O}\left(10^{-3}\right)$. Although there are several charged vectors, whose contributions add up, we find that the total effect is less than $1 \%$ of that of the $W$ gauge boson in the SM. Next we consider the corrections from a loop with a SM- $W$ and a heavy charged vector. The product of the non-diagonal couplings $Z W W_{n}$ and $h W W_{n}$ are suppressed by a factor $\lesssim \mathcal{O}\left(10^{-2}\right)$ compared with the SM coupling, thus they can be neglected as well. For the non-diagonal contributions involving heavy fields the product of the couplings $Z W_{m} W_{n}$ and $h W_{m} W_{n}$ can be of the same order as in SM. However, as in the diagonal contribution, in this case there is also an extra factor $\left(m_{W} / m_{W_{n}}\right)^{2} \sim \mathcal{O}\left(10^{-3}\right)$. Therefore, the leading correction to $h Z_{\gamma}$ mediated by loops of vector bosons is captured by the correction to the couplings $h W^{+} W^{-}$ and $Z W^{+} W^{-}$. The correction to the first one can be approximated by $F_{2}(\epsilon)=\sqrt{1-\epsilon^{2}}$, 

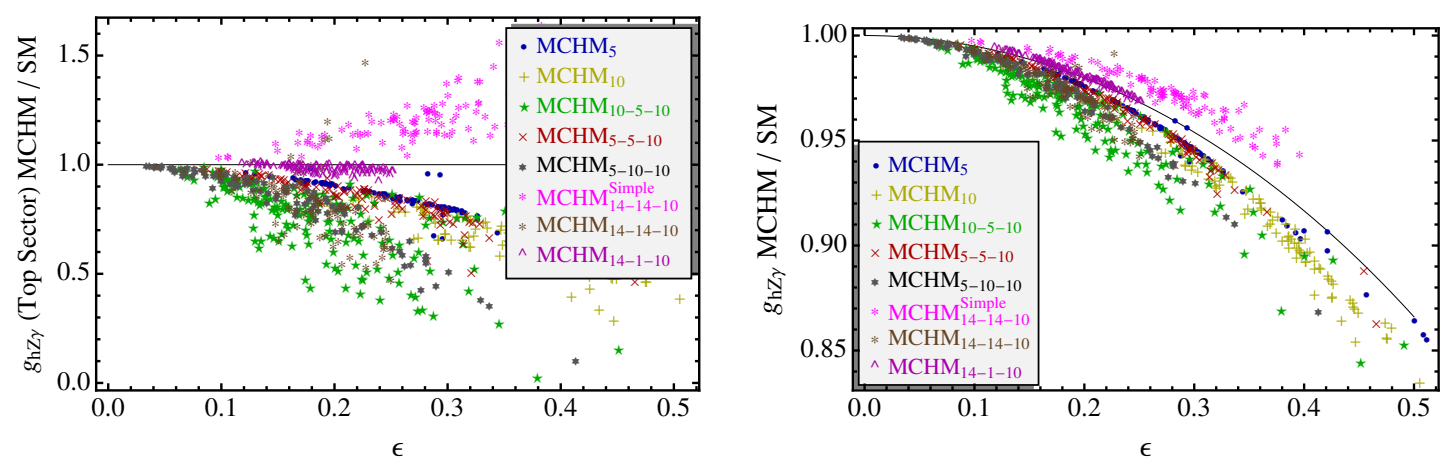

Figure 8. Left panel: the amplitude for $h \rightarrow Z \gamma$ due to the top sector in all the models, normalized to the top-mediated amplitude in the SM. Right panel: the full amplitude (absolute value), arising from vector bosons and fermions in the models of section 3, normalized to the $h Z \gamma$ amplitude in the SM. The continuous line corresponds to the SM-W loop, with its modified coupling to the Higgs as encoded in $F_{2}(\epsilon)$.

whereas the correction to $Z W^{+} W^{-}$is very small. Thus, one can expect the bosonic 1-loop correction to $c_{h Z \gamma}$ [see comments after eq. (4.10)] to be modulated by $F_{2}(\epsilon)$, leading to a suppression in the amplitude compared with the SM.

The correction from the fermionic sector is dominated by the top quark and its partners. The resonances associated to the light SM fermions decouple and do not contribute. This can be understood from the fact that $h Z \gamma$ requires breaking of $P_{L R}$, and in the present models that breaking arises only from the mixing between the two sectors of the theory. Since we are assuming that the light fermions have small mixing for both chiralities, the explicit $P_{L R}$ breaking is suppressed by these small mixings. The effect from the top partners can have different signs for different representations. In the left panel of figure 8. we show the corrections to the amplitude coming from the top sector of all the models, normalized to the top contribution in the SM. We have included all the diagonal and non-diagonal contributions. The corrections to the SM top result can be of order $50 \%$, or even larger for $\epsilon \sim 0.5$ and for most of the models there is a suppression. However one should remember that the bosonic contribution is one order of magnitude larger that the fermionic one.

In the right panel of figure 8, we show the total amplitude in the MCHM models normalized to the SM, where we have used the full diagonalization of the mass matrices and couplings to take into account all the fermionic contributions, the diagonal spin-1 contributions, and the (small) modification of the $Z W^{+} W^{-}$coupling. However, we do not include the non-diagonal gauge contributions (which have been argued to be negligible above). See appendix D for further details of this computation.

Since for most of the models and regions of parameter space the leading order effect is captured by the lightest states running in the loop, either bosons or fermions, the corrections to $c_{h Z_{\gamma}}$ can be approximated by the corrections to the Higgs couplings with $W$ and $t$. The left panel of figure 9 shows that this approximation works rather well for the models we are considering. The deviations arise mainly from the diagonal and non-diagonal contributions of the top partners. In the right panel we exhibit the correlation between the rates into $Z \gamma$ and $\gamma \gamma$. We see that this correlation is slightly different between the 

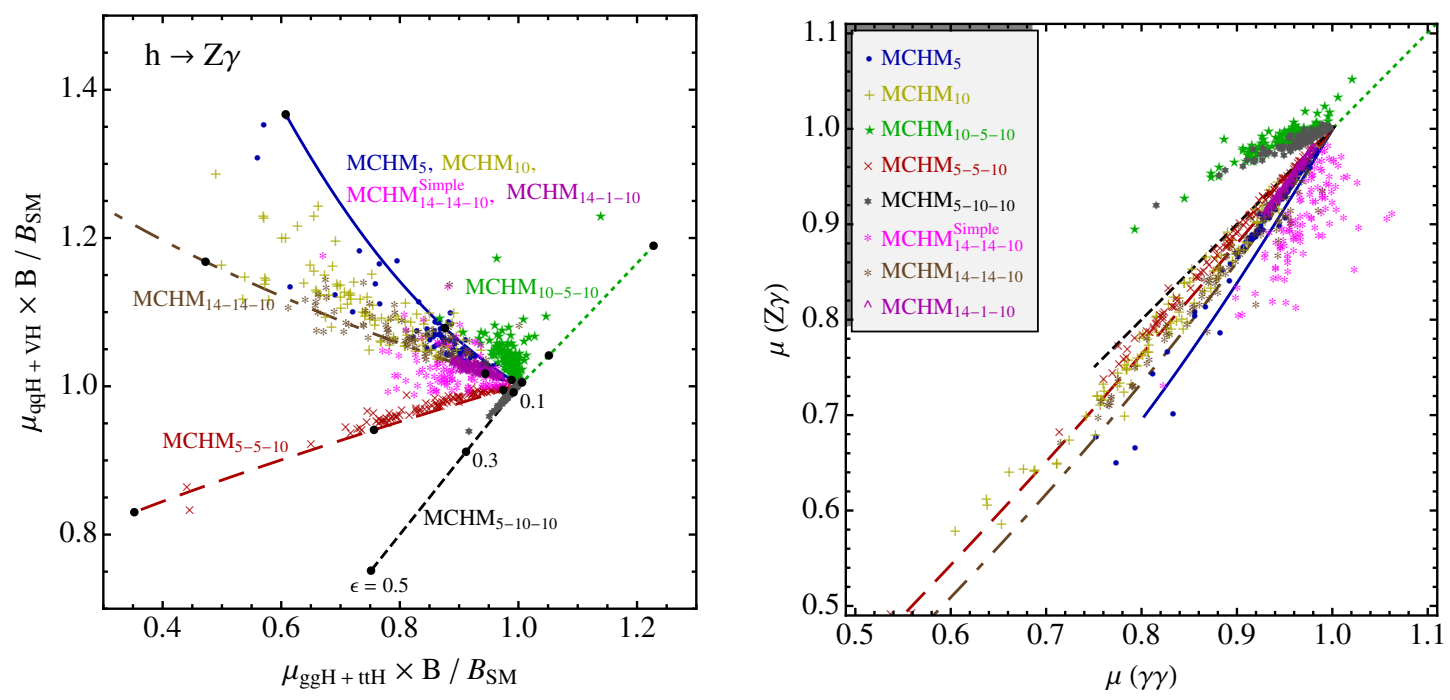

Figure 9. Left panel: similar to figure 6, but for the $h \rightarrow Z \gamma$ channel. Right panel: we show the total signal strength $\mu(i)=\sigma(p p \rightarrow h \rightarrow i) / S M$ (i.e. inclusive production) in the $Z \gamma$ channel versus the $\gamma \gamma$ channel, showing a high degree of correlation. The larger deviations from $(1,1)$ correspond to larger values of $\epsilon$.

$\mathrm{MCHM}_{10-5-10}$ and $\mathrm{MCHM}_{5-10-10}$ on the one hand, and the other models on the other, which could allow for a distinction if sufficient precision is achieved, depending on the size of the deviations from the SM. We note that for the $\mathrm{MCHM}_{5-10-10}$ there is a very good agreement between the analytical approximation and the full numerical result in $\gamma \gamma$. However, the top sector gives contributions of order $5-10 \%$ to $Z \gamma$, as can be seen in the right panel of figure 8 . Those corrections lead to the small disagreement between the analytical approximation and the full numerical result for the $\mathrm{MCHM}_{5-10-10}$ seen in the right panel of figure 9 (a similar effect but in the opposite direction is present for the $\mathrm{MCHM}_{14-14-10}^{\text {simple }}$ ).

\section{Tuning in the MCHM}

In this section we comment on the degree of fine-tuning associated with the phenomenologically viable points found above. Consistency with the EW precisions tests (EWPT) in these models, mainly the $S$-parameter and the $Z b_{L} \bar{b}_{L}$ coupling, require $\epsilon \lesssim 0.3$ [18]. ${ }^{17}$ However the Higgs potential generically leads to no EWSB, $\epsilon=0$, or to maximal EWSB, $\epsilon=1 .{ }^{18}$ A careful analysis of the structure of the Higgs potential shows that the MCHM requires some tuning in the parameter space of the theory to produce $\epsilon \lesssim 0.5$, and the amount of tuning depends on the fermion embedding [18, 54]. Besides these conditions, the Higgs potential must also lead to a light Higgs. Since the top contribution to the 1-loop

\footnotetext{
${ }^{17}$ Although we do not perform a detailed analysis of the EWPT on all the models we consider, we recall that the presence of light fermionic resonances can play an important role in opening up the viable region of parameter space, as studied in [53].

${ }^{18}$ For $v=f_{h}$, besides the problems with EWPT, many models lead to massless SM fermions, as can be seen from the cancellation of the $L R$ correlator $M_{\psi}$. This is a consequence of the restoration of an accidental chiral symmetry [55].
} 
Higgs potential is cut off by the fermionic resonances mixing with the top, a light Higgs prefers light top partners. Ref. [55] has shown the correlation between $m_{h}$ and the mass of the lightest resonance for $\mathrm{MCHM}_{5}$ and $\mathrm{MCHM}_{10}$. Ref. [54] has also discussed the impact of light fermions in the tuning of the MCHM, arriving to similar results. In our setup, similar to models in a slice of $\mathrm{AdS}_{5}$, large compositeness of the SM fermions automatically lead to light custodians that can alleviate the tuning (see the discussion in 6.1). Below we show our results for the tuning of the models presented in the previous sections.

Following refs. $[54,56,57]$ we use the sensitivity parameter

$$
\Delta=\max _{i}\left|\frac{\left.\partial \log m_{Z}\right|_{\text {phys }}}{\partial \log x_{i}}\right|
$$

as a measure of fine-tuning. Here $x_{i}$ are the parameters of the effective theory and $m_{Z}$, as given in eq. (2.16), depends explicitly on $f_{h}$ and $\epsilon$, with $\epsilon$ a function of all the parameters of the theory. By $\left.m_{Z}\right|_{\text {phys }}$ we mean that we have selected a region of parameters of the theory that leads to the observed Higgs and SM masses. We have followed the procedure of ref. [54] which has shown that eq. (7.1) can be rewritten in terms of the Higgs potential, allowing for a simple calculation of $\Delta$. As explained at the beginning of section 6 , we have considered the dependence of the potential on the following parameters: the mass scale of the composite resonances $m_{\rho}$, the decay constant of the pNGB $f_{h}$, the composite proto-Yukawa couplings, the masses mixing composite fermions $m_{y}$, the fermion mixing angles $s_{\psi}$ and the ratio of gauge couplings $\tan \theta$.

We have computed the tuning of the models presented in the previous sections, evaluating $\Delta$ in those points of the parameter space that were selected after the random scan, as explained at the beginning of section 6 . We find that the gauge contribution is subdominant, and the tuning is usually dominated by the top mixings $s_{q}, s_{t}$, the Yukawa $y_{T}$ and the mixing mass $m_{y_{T}}$ when present. Below we comment on the size of the tuning for the different models and discuss some details about its parameter dependence.

We find that the $\mathrm{MCHM}_{5}$ and $\mathrm{MCHM}_{5-5-10}$ have generically $\Delta \sim 5-40$, with the sensitivity parameter dominated by $m_{y_{T}}$ and sometimes by $s_{t}$. The second model shows some regions of parameter space with $\Delta \sim 100$ as well as some points where $s_{q}$ dominates the tuning. Notice that the $\mathrm{MCHM}_{5-5-10}$ has less freedom, since there is no $m_{y_{B}}$ and the $b_{L}$ mixing is controlled by the same parameter that controls the $t_{L}$ mixing, namely $s_{q}$, whereas for the $\mathrm{MCHM}_{5}$ there are two mixing parameters, $s_{q^{d}}$ and $s_{q^{u}}$. The $\mathrm{MCHM}_{10}$ has $\Delta \sim 5-80$, although there are points with $\Delta \sim 300$. The larger tuning of the $\mathrm{MCHM}_{10}$ could be related with the Clebsch-Gordan coefficient $\sqrt{2}$ suppressing $m_{t}$ in the latter model, that requires larger mixing and Yukawa coupling. In this model $\Delta$ is usually dominated by $s_{q}$ and sometimes by $s_{t}, y_{T}$ or $m_{y_{T}}$. As explained in the previous sections, $\mathrm{MCHM}_{5-10-10}$ and $\mathrm{MCHM}_{10-5-10}$ require a large degree of compositeness of at least one of the chiralities of the top, leading to the largest tuning of the models that we have studied with fermions in representations 5 and 10. We find $\Delta \sim 100-1000$, usually dominated by $s_{q}$ and sometimes by $s_{t}$. $\mathrm{MCHM}_{14-14-10}^{\text {simple }}$ and $\mathrm{MCHM}_{14-1-10}$ have $\Delta \sim 80-300$, dominated by $s_{t}$ for the first model and by $s_{q}$ for the second one. The main reason for the larger tuning of these models compared with $\mathrm{MCHM}_{5}$ and $\mathrm{MCHM}_{10}$ is that they generically predict a larger $m_{h}$ [54]. 
Thus, requiring $m_{h} \simeq 125 \mathrm{GeV}$ selects special regions of the parameter space with nonnatural cancellations in the Higgs potential. On the other hand, for the $\mathrm{MCHM}_{14-14-10}$ that has an extra proto-Yukawa coupling in the top sector, we find $\Delta \sim 10-150$, with the tuning dominated by $s_{t}$ and sometimes by $m_{y_{t}}$ or $f_{h}$.

We find that, after applying our selection criteria over the random scan, the models with larger tuning also show many points in a region of the parameter space with large composite scale, $f_{h} \gtrsim 4 \mathrm{TeV}$. In fact, for these models there are some points where the tuning is dominated by $f_{h}$.

\section{Conclusions}

We have used a simple two-site realization of the composite Higgs scenario [28] to systematically investigate the consequences of several fermion representations of the spontaneously broken symmetry leading to the Higgs as a pNGB. We have restricted ourselves to the $\mathrm{SO}(5) \rightarrow \mathrm{SO}(4)$ symmetry breaking pattern, which is denoted here as the "Minimal Composite Higgs Model", but we have explored several combination of the lowest-dimensional representations of $\mathrm{SO}(5)$ in the composite fermion sector. In particular, we have fully taken into account the dynamically generated Higgs potential, which receives crucial contributions from the states associated with the third family, especially the top quark. We can therefore consistently incorporate the measured mass of the resonance discovered at the LHC in 2012, interpreted as a SM-like Higgs boson, and investigate the restrictions imposed by the experimental information. We have also taken into account the effects of the bottom quark sector, which, although subdominant in determining the dynamics of EWSB, can have a non-negligible effect on the resulting Higgs phenomenology. We have assumed that the light families are mostly elementary, and therefore have a negligible effect on the Higgs potential. However, the couplings of a composite Higgs to all fermions can receive sizeable corrections leading to important deviations from the SM expectations. This can be important in the near future, as decays such as those into a $\tau$ pair are being measured with better precision $[51,52]$.

By including the "first level" of heavy (spin-1 and spin-1/2) resonances, we can also compute in detail the effects on loop-induced processes, such as the Higgs production through gluon fusion and the Higgs decays into $\gamma \gamma$ and $Z \gamma$. Such processes consist of two conceptually different, but related parts. First, the couplings of the Higgs to the SM fermions are modified w.r.t. the SM, and therefore when they run in the loop the corresponding contribution is different from the SM one. Second, the heavy resonances give an additional non-SM contribution to the loop diagrams. At zeroth-order and in the simplest models, the sum of the two effects for the dominant contributions (from the top-related states, as well as from the W-related ones in the case of $\gamma \gamma$ or $Z \gamma$ ) results in a "universal modification" that depends on the microscopic parameters only through $\epsilon=\sin v / f_{h}$. However, we find that the corrections to this leading order result, in particular those of the bottom sector, can have a qualitative impact on the Higgs properties.

Importantly, we find a generic suppression of the gluon fusion process in all the models we investigated. This is also the case for the $\mathrm{MCHM}_{14-14-10}$, which presents a richer 
structure of invariants and leads in general to a sum rule that has dependence on microscopic parameters beyond $\epsilon$. Although a priori there exists the potential for finding regions of parameter space with an enhanced gluon fusion Higgs production cross section [36], we find that all the phenomenologically viable points exhibit a rather significant suppression instead.

Due to the generic suppression of the various decay widths, in particular $\Gamma(h \rightarrow \bar{b} b)$ which dominates the total Higgs decay width, one can often find branching fractions that are larger than those in the SM. The experimental rates then result from competing effects between production and decay, and can present enhancements or suppressions in given channels, depending on the model under consideration. This offers an interesting handle -were a robust deviation from the SM to be established- to get indirect information about the composite fermion representations, which would constrain the nature of the underlying strongly interacting theory.

Another interesting decay channel is $h \rightarrow Z \gamma$. We have shown that the deviations are small and dominated by the corrections from loops of SM weak bosons, as expected if the $P_{L R}$ symmetry is not broken by the composite sector [40]. Moreover, the contributions from the heavy resonances are small and the deviations can be approximated at leading order by the corrections to the $h W^{+} W^{-}$coupling, that are given by a simple function of $\epsilon$.

We have also investigated the degree of fine-tuning, which is in general considerable but seems in most cases to compare favorably against the simplest SUSY scenarios (although this statement should not be taken as a rigorous one, given the lack of a proper UV completion for the composite Higgs scenarios). Interestingly, we find examples where the sensitivity of the weak scale to the underlying model parameters is below $10 \%$. However, models such as the $\mathrm{MCHM}_{5-10-10}$ and the $\mathrm{MCHM}_{10-5-10}$ present a sensitivity at the few per mille level. We also note that the models based on the 14 representation, which have been claimed to present little tuning [54] actually are tuned at the per cent or worse level (although we have not considered a purely composite $t_{R}$ ). These considerations may be suggestive of which case is more likely to be realized in nature, although of course experimentally the approach should be open-minded.

As the LHC and the experimental collaborations prepare for the (close to) $14 \mathrm{TeV}$ and higher luminosity run, the Higgs sector offers a unique window into physics beyond the SM. The possibility that the Higgs boson is a pNGB of some underlying strong dynamics remains as an attractive framework for understanding the breaking of the EW symmetry, and the opportunity of learning something about the detailed properties of such a theory from Higgs measurements can be a realistic one, as illustrated in this work. Eventually one should be able to produce the strong resonances, studying their properties directly, and start cross-checking against the previous low-energy information.

\section{Acknowledgments}

We thank Carlos Wagner for enlightening discussions in the beginning of this work and Álex Pomarol for useful suggestions. We also thank Abdelhak Djouadi, Joseph Lykken, Giuliano Panico, Gilad Perez and Francesco Riva for discussions. E.P. and L.D. wish 
to thank the Fermilab Theory Group for hospitality during various stages of this work. M.C. would like to thank the Aspen Center for Physics, where part of this work was completed. M.C. would like to thank ICTP-SAIFR and Centro Atómico Bariloche for hospitality. M.C. and E.P. would like to thank the MITP at the Johannes Gutenberg-University Mainz, where part of this work was completed. Fermilab is operated by Fermi Research Alliance, LLC under contract no. DE-AC02-07CH11359 with the United States Department of Energy. L.D. is partly supported by FONCYT-Argentina under the contract PICT-20101737 and CONICET-Argentina under the contract PIP 114220100100319. This work was supported by the São Paulo Research Foundation (FAPESP) under grant \# 2011/11973.

\section{A Representations of $\mathrm{SO}(5)$}

We consider the following $5 \times 5$ matrix representation of the generators $T^{B}$ of $\mathrm{SO}(5)$ :

$$
\begin{aligned}
& T_{L}^{1}=\left(\begin{array}{ccccc}
0 & 0 & 0 & -\frac{i}{2} & 0 \\
0 & 0 & -\frac{i}{2} & 0 & 0 \\
0 & \frac{i}{2} & 0 & 0 & 0 \\
\frac{i}{2} & 0 & 0 & 0 & 0 \\
0 & 0 & 0 & 0 & 0
\end{array}\right), \quad T_{L}^{2}=\left(\begin{array}{ccccc}
0 & 0 & \frac{i}{2} & 0 & 0 \\
0 & 0 & 0 & -\frac{i}{2} & 0 \\
-\frac{i}{2} & 0 & 0 & 0 & 0 \\
0 & \frac{i}{2} & 0 & 0 & 0 \\
0 & 0 & 0 & 0 & 0
\end{array}\right), \quad T_{L}^{3}=\left(\begin{array}{ccccc}
0 & -\frac{i}{2} & 0 & 0 & 0 \\
\frac{i}{2} & 0 & 0 & 0 & 0 \\
0 & 0 & 0 & -\frac{i}{2} & 0 \\
0 & 0 & \frac{i}{2} & 0 & 0 \\
0 & 0 & 0 & 0 & 0
\end{array}\right), \\
& T_{R}^{1}=\left(\begin{array}{ccccc}
0 & 0 & 0 & \frac{i}{2} & 0 \\
0 & 0 & -\frac{i}{2} & 0 & 0 \\
0 & \frac{i}{2} & 0 & 0 & 0 \\
-\frac{i}{2} & 0 & 0 & 0 & 0 \\
0 & 0 & 0 & 0 & 0
\end{array}\right), \quad T_{R}^{2}=\left(\begin{array}{ccccc}
0 & 0 & \frac{i}{2} & 0 & 0 \\
0 & 0 & 0 & \frac{i}{2} & 0 \\
-\frac{i}{2} & 0 & 0 & 0 & 0 \\
0 & -\frac{i}{2} & 0 & 0 & 0 \\
0 & 0 & 0 & 0 & 0
\end{array}\right), \quad T_{R}^{3}=\left(\begin{array}{ccccc}
0 & -\frac{i}{2} & 0 & 0 & 0 \\
\frac{i}{2} & 0 & 0 & 0 & 0 \\
0 & 0 & 0 & \frac{i}{2} & 0 \\
0 & 0 & -\frac{i}{2} & 0 & 0 \\
0 & 0 & 0 & 0 & 0
\end{array}\right), \\
& T^{\hat{1}}=\left(\begin{array}{ccccc}
0 & 0 & 0 & 0 & -\frac{i}{\sqrt{2}} \\
0 & 0 & 0 & 0 & 0 \\
0 & 0 & 0 & 0 & 0 \\
0 & 0 & 0 & 0 & 0 \\
\frac{i}{\sqrt{2}} & 0 & 0 & 0 & 0
\end{array}\right), \quad T^{\hat{2}}=\left(\begin{array}{ccccc}
0 & 0 & 0 & 0 & 0 \\
0 & 0 & 0 & 0 & -\frac{i}{\sqrt{2}} \\
0 & 0 & 0 & 0 & 0 \\
0 & 0 & 0 & 0 & 0 \\
0 & \frac{i}{\sqrt{2}} & 0 & 0 & 0
\end{array}\right) \\
& T^{\hat{3}}=\left(\begin{array}{ccccc}
0 & 0 & 0 & 0 & 0 \\
0 & 0 & 0 & 0 & 0 \\
0 & 0 & 0 & 0 & -\frac{i}{\sqrt{2}} \\
0 & 0 & 0 & 0 & 0 \\
0 & 0 & \frac{i}{\sqrt{2}} & 0 & 0
\end{array}\right), \quad T^{\hat{4}}=\left(\begin{array}{ccccc}
0 & 0 & 0 & 0 & 0 \\
0 & 0 & 0 & 0 & 0 \\
0 & 0 & 0 & 0 & 0 \\
0 & 0 & 0 & 0 & -\frac{i}{\sqrt{2}} \\
0 & 0 & 0 & \frac{i}{\sqrt{2}} & 0
\end{array}\right) .
\end{aligned}
$$

The generators $T^{B}$ act on the fundamental representation 5 of $\mathrm{SO}(5)$ as: $T^{B} \psi_{\mathbf{5}}$. One can label the components of a 5 by their transformation properties under $T_{L}^{3}$ and $T_{R}^{3}$. The 
following are eigenvectors of those generators:

$$
\begin{array}{ll}
v_{(--)}=\frac{1}{\sqrt{2}}\left(\begin{array}{c}
i \\
1 \\
0 \\
0 \\
0
\end{array}\right), & v_{(-+)}=\frac{1}{\sqrt{2}}\left(\begin{array}{l}
0 \\
0 \\
i \\
1 \\
0
\end{array}\right), \\
v_{(+-)}=\frac{1}{\sqrt{2}}\left(\begin{array}{c}
0 \\
0 \\
-i \\
1 \\
0
\end{array}\right), & v_{(++)}=\frac{1}{\sqrt{2}}\left(\begin{array}{c}
-i \\
1 \\
0 \\
0 \\
0
\end{array}\right), \quad v_{(00)}=\left(\begin{array}{l}
0 \\
0 \\
0 \\
0 \\
1
\end{array}\right),
\end{array}
$$

with the subindices $(i, j)$ labeling the $T_{L, R}^{3}$ value, \pm for $\pm 1 / 2$. Thus, a fermion $\psi$ in the fundamental representation can be written as:

$$
\psi_{\mathbf{5}}=\psi_{i} v_{(i)}=\frac{1}{\sqrt{2}}\left(\begin{array}{c}
i\left(\psi_{--}-\psi_{++}\right) \\
\psi_{--}+\psi_{++} \\
i\left(\psi_{-+}-\psi_{+-}\right) \\
\left.\psi_{-+}+\psi_{+-}\right) \\
\sqrt{2} \psi_{00}
\end{array}\right)
$$

By using the $5 \times 5$ matrix representation, the generators $T^{B}$ act on the adjoint representation 10 of $\mathrm{SO}(5)$ as: $T^{B} \psi_{\mathbf{1 0}}=\left[T^{B}, \psi_{\mathbf{1 0}}\right]$. The matrices defined in eq. (A.1) provide a basis for this representation. Other useful basis is one which can be labeled by the $T_{L, R}^{3}$ eigenvalues $v_{\left(t_{L}^{3}, t_{R}^{3}\right)}$. Since a $\mathbf{1 0}$ decomposes under $\mathrm{SO}(4) \simeq \mathrm{SU}(2)_{L} \times \mathrm{SU}(2)_{R}$ as $\mathbf{1 0} \sim(\mathbf{3}, \mathbf{1}) \oplus(\mathbf{1}, \mathbf{3}) \oplus(\mathbf{2}, \overline{\mathbf{2}})$, we obtain:

$$
\begin{aligned}
& (\mathbf{3}, \mathbf{1}): \quad v_{( \pm 1,0)}=\frac{1}{\sqrt{2}}\left(T_{L}^{1} \pm i T_{L}^{2}\right), \quad v_{(0,0)}=T_{L}^{3}, \\
& (\mathbf{1}, \mathbf{3}): \quad v_{(0, \pm 1)}=\frac{1}{\sqrt{2}}\left(T_{R}^{1} \pm i T_{R}^{2}\right), \quad v_{(0,0)}=T_{R}^{3}, \\
& (\mathbf{2}, \mathbf{2}): \quad v_{(-1 / 2,-1 / 2)}=\frac{1}{\sqrt{2}}\left(T^{\hat{1}}-i T^{\hat{2}}\right), \quad v_{(+1 / 2,+1 / 2)}=\frac{1}{\sqrt{2}}\left(T^{\hat{1}}+i T^{\hat{2}}\right), \\
& v_{(-1 / 2,+1 / 2)}=\frac{1}{\sqrt{2}}\left(T^{\hat{3}}-i T^{\hat{4}}\right), \quad v_{(+1 / 2,-1 / 2)}=\frac{1}{\sqrt{2}}\left(T^{\hat{3}}+i T^{\hat{4}}\right) .
\end{aligned}
$$

A field in the adjoint of $\mathrm{SO}(5)$ can be written as:

$$
\psi_{\mathbf{1 0}}=\psi_{i} v_{(i)}
$$

Similar to the $\mathbf{1 0}$ representation, the $\mathbf{1 4}$ representation of $\mathrm{SO}(5)$ can be written in terms of a $5 \times 5$ symmetric and traceless matrix. $T^{B}$ acts on the $\mathbf{1 4}$ as: $T^{B} \psi_{\mathbf{1 4}}=\left[T^{B}, \psi_{\mathbf{1 4}}\right]$. A 14 decomposes under $\mathrm{SU}(2)_{L} \times \mathrm{SU}(2)_{R}$ as $\mathbf{1 4} \sim(\mathbf{3}, \overline{\mathbf{3}})+(\mathbf{2}, \overline{\mathbf{2}})+(\mathbf{1}, \mathbf{1})$. One basis for this 
representation is

$$
\begin{aligned}
& (\mathbf{3}, 3): \quad T_{i j}^{a b}=\frac{1}{\sqrt{2}}\left(\delta_{i}^{a} \delta_{j}^{b}+\delta_{j}^{a} \delta_{i}^{b}\right), \quad a<b, a, b=1, \ldots 4, \\
& T_{i j}^{a a}=\frac{1}{\sqrt{2}}\left(\delta_{i}^{a} \delta_{j}^{a}-\delta_{i}^{a+1} \delta_{j}^{a+1}\right), \quad a=1,2,3, \\
& (\mathbf{2}, \mathbf{2}): \quad T_{i j}^{\hat{a}}=\frac{1}{\sqrt{2}}\left(\delta_{i}^{a} \delta_{j}^{5}+\delta_{j}^{a} \delta_{i}^{5}\right), \quad a=1, \ldots 4, \\
& (\mathbf{1}, \mathbf{1}): \quad T_{i j}^{0}=\frac{1}{2 \sqrt{5}} \operatorname{diag}(1,1,1,1,-4) .
\end{aligned}
$$

Using this basis one can define a new one labeled by the $T_{L, R}^{3}$ eigenvalues: $\left\{v_{\left(t_{L}^{3}, t_{R}^{3}\right)}\right\}$,

$(3,3):$

$$
\begin{aligned}
v_{(1,1)} & =\frac{1}{2 \sqrt{2}}\left(2 i T^{12}+T^{11}-T^{22}\right), & v_{(1,0)} & =\frac{1}{2}\left(-T^{13}-i T^{23}-i T^{14}+T^{24}\right), \\
v_{(1,-1)} & =\frac{1}{2 \sqrt{2}}\left(2 i T^{34}+T^{33}\right), & v_{(0,1)} & =\frac{1}{2}\left(-T^{13}-i T^{23}+i T^{14}-T^{24}\right), \\
v_{(0,0)} & =\frac{1}{2 \sqrt{2}}\left(-T^{11}-T^{22}+T^{33}\right), & v_{(0,-1)} & =\frac{1}{2}\left(T^{13}-i T^{23}+i T^{14}+T^{24}\right), \\
v_{(-1,1)} & =\frac{1}{2 \sqrt{2}}\left(-2 i T^{34}+T^{33}\right), & v_{(-1,0)} & =\frac{1}{2}\left(T^{13}-i T^{23}-i T^{14}-T^{24}\right), \\
v_{(-1,-1)} & =\frac{1}{2 \sqrt{2}}\left(-2 i T^{12}+T^{11}-T^{22}\right), & &
\end{aligned}
$$

$(2,2):$

$$
\begin{array}{ll}
v_{(+1 / 2,+1 / 2)}=\frac{1}{\sqrt{2}}\left(-T^{\hat{1}}-i T^{\hat{2}}\right), & v_{(+1 / 2,-1 / 2)}=\frac{1}{\sqrt{2}}\left(T^{\hat{3}}+i T^{\hat{4}}\right), \\
v_{(-1 / 2,+1 / 2)}=\frac{1}{\sqrt{2}}\left(T^{\hat{3}}-i T^{\hat{4}}\right), & v_{(-1 / 2,-1 / 2)}=\frac{1}{\sqrt{2}}\left(T^{\hat{1}}-i T^{\hat{2}}\right),
\end{array}
$$

$(1,1):$

$$
v_{(0,0)}^{\prime}=T^{0} \text {. }
$$

A field in the $\mathbf{1 4}$ representation can be written as

$$
\psi_{\mathbf{1 4}}=\psi_{i} v_{(i)}
$$

\section{B Bosonic mass matrices}

The charged (squared) mass matrix (in the basis $\left\{w_{L}^{+}, A_{L}^{+}, A_{R}^{+}, A^{\hat{+}}\right\}$ versus $\left\{w_{L}^{-}, A_{L}^{-}, A_{R}^{-}, A^{\text {无}}\right\}$, where $w_{L}^{ \pm}=\left(w_{L}^{1} \mp i w_{L}^{2}\right) / \sqrt{2}$, etc. $)$, is

$$
M_{C}^{2}=\left(\begin{array}{cccc}
\frac{1}{2} g_{0}^{2} f_{\Omega}^{2} & -\frac{1}{2} g_{0} g_{\rho} f_{\Omega}^{2} & 0 & 0 \\
-\frac{1}{2} g_{0} g_{\rho} f_{\Omega}^{2} & \frac{1}{4} g_{\rho}^{2}\left(2 f_{\Omega}^{2}+f_{1}^{2} s_{h}^{2}\right) & -\frac{1}{4} g_{\rho}^{2} f_{1}^{2} s_{h}^{2} & \frac{g_{\rho}^{2} f_{1}^{2} s_{h} c_{h}}{2 \sqrt{2}} \\
0 & -\frac{1}{4} g_{\rho}^{2} f_{1}^{2} s_{h}^{2} & \frac{1}{4} g_{\rho}^{2}\left(2 f_{\Omega}^{2}+f_{1}^{2} s_{h}^{2}\right) & -\frac{g_{\rho}^{2} f_{1}^{2} s_{h} c_{h}}{2 \sqrt{2}} \\
0 & \frac{g_{\rho}^{2} f_{1}^{2} s_{h} c_{h}}{2 \sqrt{2}} & -\frac{g_{\rho}^{2} f_{1}^{2} s_{h} c_{h}}{2 \sqrt{2}} & \frac{1}{2} g_{\rho}^{2}\left(f_{\Omega}^{2}+f_{1}^{2} c_{h}^{2}\right)
\end{array}\right),
$$


and the neutral (squared) mass matrix (in the basis $\left\{w_{L}^{3}, b, A_{L}^{3}, A_{R}^{3}, A^{\hat{3}}, A^{\hat{4}}, X\right\}$ ) is

$$
\left(\begin{array}{ccccccc}
\frac{g_{0}^{2}}{2} f_{\Omega}^{2} & 0 & -\frac{g_{0} g_{\rho}}{2} f_{\Omega}^{2} & 0 & 0 & 0 & 0 \\
0 & \frac{g_{0}^{2} g_{x}^{2}\left(f_{\Omega}^{2}+f_{\Omega_{X}}^{2}\right)}{2\left(g_{0}^{2}+g_{x}^{2}\right)} & 0 & -\frac{g_{0} g_{x} g_{\rho} f_{\Omega}^{2}}{2 \sqrt{g_{0}^{2}+g_{x}^{2}}} & 0 & 0 & -\frac{g_{0} g_{x} g_{X} f_{\Omega_{X}}^{2}}{2 \sqrt{g_{0}^{2}+g_{x}^{2}}} \\
-\frac{g_{0} g_{\rho}}{2} f_{\Omega}^{2} & 0 & \frac{g_{\rho}^{2}}{2}\left(f_{\Omega}^{2}+\frac{f_{1}^{2} s_{h}^{2}}{2}\right) & -\frac{g_{\rho}^{2}}{4} f_{1}^{2} s_{h}^{2} & \frac{g_{\rho}^{2} f_{1}^{2} s_{h} c_{h}}{2 \sqrt{2}} & 0 & 0 \\
0 & -\frac{g_{0} g_{x} g_{\rho} f_{\Omega}^{2}}{2 \sqrt{g_{0}^{2}+g_{x}^{2}}} & -\frac{g_{\rho}^{2}}{4} f_{1}^{2} s_{h}^{2} & \frac{g_{\rho}^{2}}{2}\left(f_{\Omega}^{2}+\frac{f_{1}^{2} s_{h}^{2}}{2}\right) & -\frac{g_{\rho}^{2} f_{1}^{2} s_{s} c_{h}}{2 \sqrt{2}} & 0 & 0 \\
0 & 0 & \frac{g_{\rho}^{2} f_{1}^{2} s_{h} c_{h}}{2 \sqrt{2}} & -\frac{g_{\rho}^{2} f_{1}^{2} s_{h} c_{h}}{2 \sqrt{2}} & \frac{g_{\rho}^{2}}{2}\left(f_{\Omega}^{2}+f_{1}^{2} c_{h}^{2}\right) & 0 & 0 \\
0 & 0 & 0 & 0 & 0 & \frac{g_{\rho}^{2}}{2}\left(f_{\Omega}^{2}+f_{1}^{2}\right) & 0 \\
0 & -\frac{g_{0} g_{x} g_{X} f_{\Omega_{X}}^{2}}{2 \sqrt{g_{0}^{2}+g_{x}^{2}}} & 0 & 0 & 0 & 0 & \frac{g_{X}^{2}}{2} f_{\Omega_{X}}^{2}
\end{array}\right) .
$$

\section{Correlators}

In this appendix we express the fermionic correlators of all the models in the $\mathrm{SO}(4)$ symmetric phase in terms of the following general functions.

$$
\begin{aligned}
A_{L}\left(m_{1}, m_{2}, m_{3}, m_{4}, \Delta\right)= & \Delta^{2}\left[m_{1}^{2} m_{2}^{2}+m_{1}^{2} m_{4}^{2}+m_{2}^{2} m_{3}^{2}\right. \\
& \left.-p^{2}\left(m_{1}^{2}+m_{2}^{2}+m_{3}^{2}+m_{4}^{2}\right)+p^{4}\right] ; \\
A_{R}\left(m_{1}, m_{2}, m_{3}, m_{4}, \Delta\right)= & \Delta^{2}\left[m_{1}^{2} m_{2}^{2}+m_{2}^{2} m_{3}^{2}-p^{2}\left(m_{1}^{2}+m_{2}^{2}+m_{3}^{2}+m_{4}^{2}\right)+p^{4}\right] ; \\
A_{M}\left(m_{1}, m_{2}, m_{3}, m_{4}, \Delta_{1}, \Delta_{2}\right)= & \Delta_{1} \Delta_{2} m_{1} m_{2} m_{4}\left(m_{3}^{2}-p^{2}\right) ; \\
B\left(m_{1}, m_{2}, m_{3}, m_{4}, m_{5}\right)= & m_{1}^{2} m_{2}^{2} m_{3}^{2}-p^{2}\left(m_{1}^{2} m_{2}^{2}+m_{1}^{2} m_{3}^{2}+m_{2}^{2} m_{3}^{2}+m_{2}^{2} m_{5}^{2}+m_{3}^{2} m_{4}^{2}\right) \\
& +p^{4}\left(m_{1}^{2}+m_{2}^{2}+m_{3}^{2}+m_{4}^{2}+m_{5}^{2}\right)-p^{6} .
\end{aligned}
$$

In the following expressions we use the notation $y_{T}=y_{u}, y_{B}=y_{d}, m_{y_{T}}=m_{y_{u}}$, $m_{y_{B}}=m_{y_{d}}, \Delta_{T}=\Delta_{u}$ and $\Delta_{B}=\Delta_{b}$ [where the Lagrangian parameters were defined for each model in section 3] to emphasize the role of the third generation.

\section{C.1 $\mathrm{MCHM}_{5}$}

$$
\begin{array}{ll}
\hat{\Pi}_{q^{u(1)}}=\frac{A_{L}\left(m_{T}, 0, m_{y_{T}}+y_{T}, 0, \Delta_{q} u\right)}{B\left(m_{Q^{u}}, m_{T}, 0, m_{y_{T}}+y_{T}, 0\right)}, & \hat{\Pi}_{q^{u(4)}}=\frac{A_{L}\left(m_{T}, 0, m_{y_{T}}, 0, \Delta_{q} u\right)}{B\left(m_{Q^{u}}, m_{T}, 0, m_{y_{T}}, 0\right)}, \\
\hat{\Pi}_{q^{d(1)}}=\frac{A_{L}\left(m_{B}, 0, m_{y_{B}}+y_{B}, 0, \Delta_{q}\right)}{B\left(m_{Q^{d}}, m_{B}, 0, m_{y_{B}}+y_{B}, 0\right)}, & \hat{\Pi}_{q^{d(4)}}=\frac{A_{L}\left(m_{B}, 0, m_{y_{B}}, 0, \Delta_{q} d\right.}{B\left(m_{Q^{d}}, m_{B}, 0, m_{y_{B}}, 0\right)}, \\
\hat{\Pi}_{u^{(1)}}=\frac{A_{R}\left(m_{Q^{u}}, 0, m_{y_{T}}+y_{T}, 0, \Delta_{t}\right)}{B\left(m_{Q^{u}}, m_{T}, 0, m_{y_{T}}+y_{T}, 0\right)}, & \hat{\Pi}_{u^{(4)}}=\frac{A_{R}\left(m_{Q^{u}}, 0, m_{y_{T}}, 0, \Delta_{t}\right)}{B\left(m_{Q^{u}}, m_{T}, 0, m_{y_{T}}, 0\right)}, \\
\hat{\Pi}_{d^{(1)}}=\frac{A_{R}\left(m_{Q^{d}}, 0, m_{y_{B}}+y_{B}, 0, \Delta_{b}\right)}{B\left(m_{Q^{d}}, m_{B}, 0, m_{y_{B}}+y_{B}, 0\right)}, & \hat{\Pi}_{d^{(4)}}=\frac{A_{R}\left(m_{Q^{d}}, 0, m_{y_{B}}, 0, \Delta_{b}\right)}{B\left(m_{Q^{d}}, m_{B}, 0, m_{y_{B}}, 0\right)}, \\
\hat{M}_{u^{(1)}}=\frac{A_{M}\left(m_{Q^{u}}, m_{T}, 0, m_{y_{T}}+y_{T}, \Delta_{q} u, \Delta_{t}\right)}{B\left(m_{Q^{u}}, m_{T}, 0, m_{y_{T}}+y_{T}, 0\right)}, & \hat{M}_{u^{(4)}}=\frac{A_{M}\left(m_{Q^{u}}, m_{T}, 0, m_{y_{T}}, \Delta_{q^{u}}, \Delta_{t}\right)}{B\left(m_{Q^{u}}, m_{T}, 0, m_{y_{T}}, 0\right)}, \\
\hat{M}_{d^{(1)}}=\frac{A_{M}\left(m_{Q^{d}}, m_{B}, 0, m_{y_{B}}+y_{B}, \Delta_{q^{d}}, \Delta_{b}\right)}{B\left(m_{Q^{d}}, m_{B}, 0, m_{y_{B}}+y_{B}, 0\right)}, \hat{M}_{d^{(4)}}=\frac{A_{M}\left(m_{Q^{d}}, m_{B}, 0, m_{y_{B}}, \Delta_{q^{d}}, \Delta_{b}\right)}{B\left(m_{Q^{d}}, m_{B}, 0, m_{y_{B}}, 0\right)},
\end{array}
$$




\section{C.2 $\mathrm{MCHM}_{10}$}

$$
\begin{aligned}
& \hat{\Pi}_{q^{(4)}}=\frac{A_{L}\left(m_{T}, m_{B}, m_{y_{T}}+y_{T} / 2, m_{y_{B}}+y_{B} / 2, \Delta_{q}\right)}{B\left(m_{Q}, m_{T}, m_{B}, m_{y_{T}}+y_{T} / 2, m_{y_{B}}+y_{B} / 2\right)}, \hat{\Pi}_{q^{(6)}}=\frac{A_{L}\left(m_{T}, m_{B}, m_{y_{T}}, m_{y_{B}}, \Delta_{q}\right)}{B\left(m_{Q}, m_{T}, m_{B}, m_{y_{T}}, m_{y_{B}}\right)}, \\
& \hat{\Pi}_{u^{(4)}}=\frac{A_{R}\left(m_{Q}, m_{B}, m_{y_{T}}+y_{T} / 2, m_{y_{B}}+y_{B} / 2, \Delta_{t}\right)}{B\left(m_{Q}, m_{T}, m_{B}, m_{y_{T}}+y_{T} / 2, m_{y_{B}}+y_{B} / 2\right)}, \hat{\Pi}_{u^{(6)}}=\frac{A_{R}\left(m_{Q}, m_{B}, m_{y_{T}}, m_{y_{B}}, \Delta_{t}\right)}{B\left(m_{Q}, m_{T}, m_{B}, m_{y_{T}}, m_{y_{B}}\right)}, \\
& \hat{\Pi}_{d^{(4)}}=\frac{A_{R}\left(m_{Q}, m_{T}, m_{y_{B}}+y_{B} / 2, m_{y_{T}}+y_{T} / 2, \Delta_{b}\right)}{B\left(m_{Q}, m_{T}, m_{B}, m_{y_{T}}+y_{T} / 2, m_{y_{B}}+y_{B} / 2\right)}, \quad \hat{\Pi}_{d^{(6)}}=\frac{A_{R}\left(m_{Q}, m_{T}, m_{y_{B}}, m_{y_{T}}, \Delta_{b}\right)}{B\left(m_{Q}, m_{T}, m_{B}, m_{y_{T}}, m_{y_{B}}\right)}, \\
& \hat{M}_{u^{(4)}}=\frac{A_{M}\left(m_{Q}, m_{T}, m_{B}, m_{y_{T}}+y_{T} / 2, \Delta_{q}, \Delta_{t}\right)}{B\left(m_{Q}, m_{T}, m_{B}, m_{y_{T}}+y_{T} / 2, m_{y_{B}}+y_{B} / 2\right)}, \quad \hat{M}_{u^{(6)}}=\frac{A_{M}\left(m_{Q}, m_{T}, m_{B}, m_{y_{T}}, \Delta_{q}, \Delta_{t}\right)}{B\left(m_{Q}, m_{T}, m_{B}, m_{y_{T}}, m_{y_{B}}\right)}, \\
& \hat{M}_{d^{(4)}}=\frac{A_{M}\left(m_{Q}, m_{B}, m_{T}, m_{y_{B}}+y_{B} / 2, \Delta_{q}, \Delta_{b}\right)}{B\left(m_{Q}, m_{T}, m_{B}, m_{y_{T}}+y_{T} / 2, m_{y_{B}}+y_{B} / 2\right)}, \quad \hat{M}_{d^{(6)}}=\frac{A_{M}\left(m_{Q}, m_{B}, m_{T}, m_{y_{B}}, \Delta_{q}, \Delta_{b}\right)}{B\left(m_{Q}, m_{T}, m_{B}, m_{y_{T}}, m_{y_{B}}\right)} .
\end{aligned}
$$

\section{C.3 $\mathrm{MCHM}_{10-5-10}$}

$$
\begin{array}{ll}
\hat{\Pi}_{q^{(4)}}=\frac{A_{L}\left(m_{T}, m_{B}, y_{T} / \sqrt{2}, m_{y_{B}}+y_{B} / 2, \Delta_{q}\right)}{B\left(m_{Q}, m_{T}, m_{B}, y_{T} / \sqrt{2}, m_{y_{B}}+y_{B} / 2\right)}, & \hat{\Pi}_{q^{(6)}}=\frac{A_{L}\left(0, m_{B}, 0, m_{y_{B}}, \Delta_{q}\right)}{B\left(m_{Q}, 0, m_{B}, 0, m_{y_{B}}\right)}, \\
\hat{\Pi}_{u^{(4)}}=\frac{A_{R}\left(m_{Q}, m_{B}, y_{T} / \sqrt{2}, m_{y_{B}}+y_{B} / 2, \Delta_{t}\right)}{B\left(m_{Q}, m_{T}, m_{B}, y_{T} / \sqrt{2}, m_{y_{B}}+y_{B} / 2\right)}, & \hat{\Pi}_{u^{(1)}}=\frac{A_{R}\left(0,0,0,0, \Delta_{t}\right)}{B\left(0, m_{T}, 0,0,0\right)}, \\
\hat{\Pi}_{d^{(4)}}=\frac{A_{R}\left(m_{Q}, m_{T}, m_{y_{B}}+y_{B} / 2, y_{T} / \sqrt{2}, \Delta_{b}\right)}{B\left(m_{Q}, m_{T}, m_{B}, y_{T} / \sqrt{2}, m_{y_{B}}+y_{B} / 2\right)}, & \hat{\Pi}_{d^{(6)}}=\frac{A_{R}\left(0, m_{Q}, 0, m_{y_{B}}, \Delta_{b}\right)}{B\left(m_{Q}, 0, m_{B}, 0, m_{y_{B}}\right.}, \\
\hat{M}_{u^{(4)}}=\frac{A_{M}\left(m_{Q}, m_{T}, m_{B}, y_{T} / \sqrt{2}, m_{y_{B}}, \Delta_{q}, \Delta_{t}\right)}{B\left(m_{Q}, m_{T}, m_{B}, y_{T} / \sqrt{2}, m_{y_{B}}+y_{B} / 2\right)}, & \\
\hat{M}_{d^{(4)}}=\frac{A_{M}\left(m_{Q}, m_{B}, m_{T}, m_{y_{B}}+y_{B} / 2, \Delta_{q}, \Delta_{b}\right)}{B\left(m_{Q}, m_{T}, m_{B}, y_{T} / \sqrt{2}, m_{y_{B}}+y_{B} / 2\right)}, & \hat{M}_{d^{(6)}}=\frac{A_{M}\left(m_{Q}, m_{B}, 0, m_{\left.y_{B}, \Delta_{q}, \Delta_{b}\right)}^{B\left(m_{Q}, 0, m_{B}, 0, m_{y_{B}}\right)} .\right.}{} .
\end{array}
$$

\section{C.4 $\mathrm{MCHM}_{5-5-10}$}

$$
\begin{aligned}
& \hat{\Pi}_{q^{(4)}}=\frac{A_{L}\left(m_{T}, m_{B}, m_{y_{T}}, y_{B} / \sqrt{2}, \Delta_{q}\right)}{B\left(m_{Q}, m_{T}, m_{B}, m_{y_{T}}, y_{B} / \sqrt{2}\right)}, \quad \hat{\Pi}_{q^{(1)}}=\frac{A_{L}\left(m_{T}, 0, m_{y_{T}}+y_{T}, 0, \Delta_{q}\right)}{B\left(m_{Q}, m_{T}, 0, m_{y_{T}}+y_{T}, 0\right)}, \\
& \hat{\Pi}_{u^{(4)}}=\frac{A_{R}\left(m_{Q}, m_{B}, m_{y_{T}}, y_{B} / \sqrt{2}, \Delta_{t}\right)}{B\left(m_{Q}, m_{T}, m_{B}, m_{y_{T}}, y_{B} / \sqrt{2}\right)}, \quad \hat{\Pi}_{u^{(1)}}=\frac{A_{R}\left(m_{Q}, 0, m_{y_{T}}+y_{T}, 0, \Delta_{t}\right)}{B\left(m_{Q}, m_{T}, 0, m_{y_{T}}+y_{T}, 0\right)}, \\
& \hat{\Pi}_{d^{(4)}}=\frac{A_{R}\left(m_{Q}, m_{T}, y_{B} / \sqrt{2}, m_{y_{T}}, \Delta_{b}\right)}{B\left(m_{Q}, m_{T}, m_{B}, m_{y_{T}}, y_{B} / \sqrt{2}\right)}, \quad \hat{\Pi}_{d^{(6)}}=\frac{A_{R}\left(0,0,0,0, \Delta_{b}\right)}{B\left(0,0, m_{B}, 0,0\right.}, \\
& \hat{M}_{u^{(4)}}=\frac{A_{M}\left(m_{Q}, m_{T}, m_{B}, m_{y_{T}}, \Delta_{q}, \Delta_{t}\right)}{B\left(m_{Q}, m_{T}, m_{B}, m_{y_{T}}, y_{B} / \sqrt{2}\right)}, \quad \hat{M}_{u^{(1)}}=\frac{A_{M}\left(m_{Q}, m_{T}, 0, m_{y_{T}}+y_{T}, \Delta_{q}, \Delta_{t}\right)}{B\left(m_{Q}, m_{T}, 0, m_{y_{T}}+y_{T}, 0\right)}, \\
& \hat{M}_{d^{(4)}}=\frac{A_{M}\left(m_{Q}, m_{B}, m_{T}, y_{B} / \sqrt{2}, \Delta_{q}, \Delta_{b}\right)}{B\left(m_{Q}, m_{T}, m_{B}, m_{y_{T}}, y_{B} / \sqrt{2}\right)} \text {. }
\end{aligned}
$$

\section{C.5 $\mathrm{MCHM}_{5-10-10}$}

$$
\begin{aligned}
& \hat{\Pi}_{q^{(4)}}=\frac{A_{L}\left(m_{T}, m_{B}, y_{T} / \sqrt{2}, y_{B} / \sqrt{2}, \Delta_{q}\right)}{B\left(m_{Q}, m_{T}, m_{B}, y_{T} / \sqrt{2}, y_{B} / \sqrt{2}\right)}, \quad \hat{\Pi}_{q^{(1)}}=\frac{A_{L}\left(0,0,0,0, \Delta_{q}\right)}{B\left(m_{Q}, 0,0,0,0\right)} \\
& \hat{\Pi}_{u^{(4)}}=\frac{A_{R}\left(m_{Q}, m_{B}, y_{T} / \sqrt{2}, y_{B} / \sqrt{2}, \Delta_{t}\right)}{B\left(m_{Q}, m_{T}, m_{B}, y_{T} / \sqrt{2}, y_{B} / \sqrt{2}\right)}, \quad \hat{\Pi}_{u^{(6)}}=\frac{A_{R}\left(0,0,0,0, \Delta_{t}\right)}{B\left(0, m_{T}, 0,0,0\right)} \\
& \hat{\Pi}_{d^{(4)}}=\frac{A_{R}\left(m_{Q}, m_{T}, y_{B} / \sqrt{2}, y_{T} / \sqrt{2}, \Delta_{b}\right)}{B\left(m_{Q}, m_{T}, m_{B}, y_{T} / \sqrt{2}, y_{B} / \sqrt{2}\right)}, \quad \hat{\Pi}_{d^{(6)}}=\frac{A_{R}\left(0,0,0,0, \Delta_{b}\right)}{B\left(0,0, m_{B}, 0,0\right)} \\
& \hat{M}_{u^{(4)}}=\frac{A_{M}\left(m_{Q}, m_{T}, m_{B}, y_{T} / \sqrt{2}, \Delta_{q}, \Delta_{t}\right)}{B\left(m_{Q}, m_{T}, m_{B}, y_{T} / \sqrt{2}, y_{B} / \sqrt{2}\right)} \\
& \hat{M}_{d^{(4)}}=\frac{A_{M}\left(m_{Q}, m_{B}, m_{T}, y_{B} / \sqrt{2}, \Delta_{q}, \Delta_{b}\right)}{B\left(m_{Q}, m_{T}, m_{B}, y_{T} / \sqrt{2}, y_{B} / \sqrt{2}\right)}
\end{aligned}
$$




\section{C.6 $\mathrm{MCHM}_{14-1-10}$}

$$
\begin{aligned}
& \hat{\Pi}_{q^{(9)}}=\frac{A_{L}\left(0,0,0,0, \Delta_{q}\right)}{B\left(m_{Q}, 0,0,0,0\right)}, \quad \hat{\Pi}_{q^{(4)}}=\frac{A_{L}\left(0, m_{B}, 0, y_{B} / 2, \Delta_{q}\right)}{B\left(m_{Q}, 0, m_{B}, 0, y_{B} / 2\right)}, \\
& \hat{\Pi}_{q^{(1)}}=\frac{A_{L}\left(m_{T}, 0, y_{T} \sqrt{4 / 5}, 0, \Delta_{q}\right)}{B\left(m_{Q}, m_{T}, 0, y_{T} \sqrt{4 / 5}, 0\right)}, \\
& \hat{\Pi}_{u^{(1)}}=\frac{A_{R}\left(m_{Q}, 0, y_{T} \sqrt{4 / 5}, 0, \Delta_{t}\right)}{B\left(m_{Q}, m_{T}, 0, y_{T} \sqrt{4 / 5}, 0\right)}, \quad \hat{\Pi}_{d^{(6)}}=\frac{A_{R}\left(0,0,0,0, \Delta_{b}\right)}{B\left(0, m_{B}, 0,0,0\right)} \\
& \hat{\Pi}_{d^{(4)}}=\frac{A_{R}\left(m_{Q}, 0, y_{B} / 2,0, \Delta_{b}\right)}{B\left(m_{Q}, 0, m_{B}, 0, y_{B} / 2\right)}, \\
& \hat{M}_{u^{(4)}}=-\frac{A_{M}\left(m_{Q}, m_{T}, 0, y_{T} \sqrt{4 / 5}, \Delta_{q}, \Delta_{t}\right)}{B\left(m_{Q}, m_{T}, 0, y_{T} \sqrt{4 / 5}, 0\right)}, \\
& \hat{M}_{d^{(4)}}=-i \frac{A_{M}\left(m_{Q}, m_{B}, 0, y_{B} / 2, \Delta_{q}, \Delta_{b}\right)}{B\left(m_{Q}, 0, m_{B}, 0, y_{B} / 2\right)},
\end{aligned}
$$

\section{C.7 $\mathrm{MCHM}_{14-14-10}$}

Here we use the notation $\bar{y}_{T}=y_{T}+\tilde{y}_{T}$ that includes the two Yukawa structures displayed in eqs. (3.36) and (3.37), which enters in the singlet terms below:

$$
\begin{array}{ll}
\hat{\Pi}_{q^{(9)}}=\frac{A_{L}\left(m_{T}, 0, m_{y_{T}}, 0, \Delta_{q}\right)}{B\left(m_{Q}, m_{T}, 0, m_{y_{T}}, 0\right)}, & \hat{\Pi}_{q^{(4)}}=\frac{A_{L}\left(m_{T}, m_{B}, m_{y_{T}}+y_{T} / 2, y_{B} / 2, \Delta_{q}\right)}{B\left(m_{Q}, m_{T}, m_{B}, m_{y_{T}}+y_{T} / 2, y_{B} / 2\right)} \\
\hat{\Pi}_{q^{(1)}}=\frac{A_{L}\left(m_{T}, 0, m_{y_{T}}+\bar{y}_{T} 4 / 5,0, \Delta_{q}\right)}{B\left(m_{Q}, m_{T}, 0, m_{y_{T}}+\bar{y}_{T} 4 / 5,0\right)}, & \hat{\Pi}_{u^{(4)}}=\frac{A_{R}\left(m_{Q}, m_{B}, m_{y_{T}}+y_{T} / 2, y_{B} / 2, \Delta_{t}\right)}{B\left(m_{Q}, m_{T}, m_{B}, m_{y_{T}}+y_{T} / 2, y_{B} / 2\right)}, \\
\hat{\Pi}_{u^{(9)}}=\frac{A_{R}\left(m_{Q}, 0, m_{y_{T}}, 0, \Delta_{t}\right)}{B\left(m_{Q}, m_{T}, 0, m_{y_{T}}, 0\right)}, & \hat{\Pi}_{u^{(1)}}=\frac{A_{R}\left(m_{Q}, 0, m_{y_{T}}+\bar{y}_{T} 4 / 5,0, \Delta_{t}\right)}{B\left(m_{Q}, m_{T}, 0, m_{y_{T}}+\bar{y}_{T} 4 / 5,0\right)}, \\
\hat{\Pi}_{d^{(4)}}=\frac{A_{R}\left(m_{Q}, m_{T}, y_{B} / 2, m_{y_{T}}+y_{T} / 2, \Delta_{b}\right)}{B\left(m_{Q}, m_{T}, m_{B}, m_{y_{T}}+y_{T} / 2, y_{B} / 2\right)}, & \hat{\Pi}_{d^{(6)}}=\frac{A_{R}\left(m_{Q}, 0,0,0, \Delta_{b}\right)}{B\left(m_{Q}, m_{B}, 0,0,0\right)}, \\
\hat{M}_{u^{(9)}}=\frac{A_{M}\left(m_{Q}, m_{T}, 0, m_{y_{T}}, \Delta_{q}, \Delta_{t}\right)}{B\left(m_{Q}, m_{T}, 0, m_{y_{T}}, 0\right)}, & \hat{M}_{u^{(4)}}=\frac{A_{M}\left(m_{Q}, m_{T}, m_{B}, m_{y_{T}}+y_{T} / 2, \Delta_{q}, \Delta_{t}\right)}{B\left(m_{Q}, m_{T}, m_{B}, m_{y_{T}}+y_{T} / 2, y_{B} / 2\right)} \\
\hat{M}_{u^{(1)}}=\frac{A_{M}\left(m_{Q}, m_{T}, 0, m_{y_{T}}+\bar{y}_{T} 4 / 5, \Delta_{q}, \Delta_{t}\right)}{B\left(m_{Q}, m_{T}, 0, m_{y_{T}}+\bar{y}_{T} 4 / 5,0\right)}, & \\
\hat{M}_{d^{(4)}}=-i \frac{A_{M}\left(m_{Q}, m_{B}, m_{T}, y_{B} / 2, \Delta_{q}, \Delta_{b}\right)}{B\left(m_{Q}, m_{T}, m_{B}, m_{y_{T}}+y_{T} / 2, y_{B} / 2\right)}, &
\end{array}
$$

\section{Loop-level processes}

We collect here the expressions for the processes $h \rightarrow g g, h \rightarrow \gamma \gamma$ and $h \rightarrow Z \gamma$. We focus on the amplitudes only, since the decay rates are obtained by rescaling the SM rates. This allows one to include the state of the art QCD corrections, under the assumption that the $\mathrm{K}$-factors for the SM and new physics diagrams are common. The full details for the SM expressions can be found, for instance, in [58].

For the $h \rightarrow g g$ and $h \rightarrow \gamma \gamma$ amplitudes the relevant loop functions are

$$
\begin{aligned}
A_{1 / 2}(\tau) & =2[\tau+(\tau-1) f(\tau)] \tau^{-2}, \\
A_{1}(\tau) & =-\left[2 \tau^{2}+3 \tau+3(2 \tau-1) f(\tau)\right] \tau^{-2},
\end{aligned}
$$


where

$$
f(\tau)=\left\{\begin{array}{ll}
\arcsin ^{2} \sqrt{\tau} & \tau \leq 1 \\
-\frac{1}{4}\left[\log \frac{1+\sqrt{1-\tau^{-1}}}{1-\sqrt{1-\tau^{-1}}}-i \pi\right]^{2} & \tau>1
\end{array} .\right.
$$

We obtain our amplitude for the gluon fusion process from

$$
\mathcal{A}(h \rightarrow g g) \propto v_{\mathrm{SM}} \sum_{\psi=t, b}\left\{\frac{4}{3}\left[\operatorname{tr}\left(Y_{\psi} M_{\psi}^{-1}\right)-\frac{y_{\psi}^{(0)}}{m_{\psi}^{(0)}}\right]+\frac{y_{\psi}^{(0)}}{m_{\psi}^{(0)}} A_{1 / 2}\left(\frac{m_{h}^{2}}{4 m_{\psi}^{(0) 2}}\right)\right\},
$$

where $y_{\psi}^{(0)}$ and $m_{\psi}^{(0)}$ are the Yukawa coupling and mass of the lightest state (identified with the SM fermion) in the corresponding tower, obtained by numerical diagonalization of the full Yukawa and mass matrices, $Y_{\psi}$ and $M_{\psi}$, respectively. The traces can be read for each model from table. 1. The SM amplitude, in the same normalization as eq. (D.4), reads $\mathcal{A}(h \rightarrow g g)^{\mathrm{SM}} \propto A_{1 / 2}\left(m_{h}^{2} / 4 m_{t}^{2}\right)+A_{1 / 2}\left(m_{h}^{2} / 4 m_{b}^{2}\right)$.

For the diphoton channel, we use

$$
\begin{aligned}
\frac{\mathcal{A}(h \rightarrow \gamma \gamma)}{v_{\mathrm{SM}}} \propto-7 & {\left[\frac{\cot \left(v / f_{h}\right)}{f_{h}}-\frac{g_{W}^{2} v_{\mathrm{SM}}}{4 m_{W}^{2}}\right]+\frac{g_{W}^{2} v_{\mathrm{SM}}}{4 m_{W}^{2}} A_{1}\left(m_{h}^{2} / 4 m_{W}^{2}\right) } \\
& +\sum_{\psi=t, b} N_{c} Q_{\psi}^{2}\left\{\frac{4}{3}\left[\operatorname{tr}\left(Y_{\psi} M_{\psi}^{-1}\right)-\frac{y_{\psi}^{(0)}}{m_{\psi}^{(0)}}\right]+\frac{y_{\psi}^{(0)}}{m_{\psi}^{(0)}} A_{1 / 2}\left(\frac{m_{h}^{2}}{4 m_{\psi}^{(0) 2}}\right)\right\}
\end{aligned}
$$

where for the $W$-tower we used that the analogue of the fermion trace is $\frac{1}{2} d \log \left(\operatorname{det} M_{C}^{2}\right) / d v=\cot \left(v / f_{h}\right) / f_{h}$ with $M_{C}^{2}$ the squared mass matrix in the charged sector [see eq. (B.1) of appendix B]. The $W$-mass squared, $m_{W}^{2}$, corresponds to the lightest eigenvalue of $M_{C}^{2}$, and the coupling $g_{W}^{2}$ is defined as the diagonal entry corresponding to this lightest state in the matrix $G_{C}^{2}=\left(2 / v_{\mathrm{SM}}\right) d M_{C}^{2} / d v$, after rotating to the mass eigenbasis. Both $m_{W}$ and $g_{W}^{2}$ are obtained numerically. The SM amplitude in the normalization of eq. (D.5) is $\mathcal{A}(h \rightarrow \gamma \gamma)^{\mathrm{SM}} \propto A_{1}\left(m_{h}^{2} / 4 m_{W}^{2}\right)+N_{c} Q_{t}^{2} A_{1 / 2}\left(m_{h}^{2} / 4 m_{t}^{2}\right)+N_{c} Q_{b}^{2} A_{1 / 2}\left(m_{h}^{2} / 4 m_{b}^{2}\right)$.

The new feature in the $h \rightarrow Z \gamma$ process compared to the previous ones [59] is that there can be two different particle species running in the loop (since the $Z$ vertex corresponds to a broken gauge symmetry). For the fermionic contributions we use the general formulas presented in appendix $\mathrm{F}$ of ref. [37], which allow to include such "non-diagonal" contributions. These expressions are written in terms of the Passarino-Veltman 1-loop functions and we use the package LoopTools [60] to evaluate them numerically.

For the charged $W$ and heavy partner loops in $h \rightarrow Z \gamma$ there is no analogue general formula for the non-diagonal contributions. Since we expect such effects to be negligible due to the large masses involved, we are satisfied with including only the diagonal gauge effects, which are completely dominated by the SM- $W$ loop itself (but the result is different from the SM one due to the modified couplings). The diagonal terms can be written in terms of

$$
A_{1}(\tau, \lambda)=4\left(3-\frac{s_{W}^{2}}{c_{W}^{2}}\right) I_{2}(\tau, \lambda)+\left[\left(1+\frac{2}{\tau}\right) \frac{s_{W}^{2}}{c_{W}^{2}}-\left(5+\frac{2}{\tau}\right)\right] I_{1}(\tau, \lambda),
$$


where

$$
\begin{aligned}
& I_{1}(\tau, \lambda)=\frac{\tau \lambda}{2(\tau-\lambda)}+\frac{\tau^{2} \lambda^{2}}{2(\tau-\lambda)^{2}}\left[f\left(\tau^{-1}\right)-f\left(\lambda^{-1}\right)\right]+\frac{\tau^{2} \lambda}{(\tau-\lambda)^{2}}\left[g\left(\tau^{-1}\right)-g\left(\lambda^{-1}\right)\right], \\
& I_{2}(\tau, \lambda)=-\frac{\tau \lambda}{2(\tau-\lambda)}\left[f\left(\tau^{-1}\right)-f\left(\lambda^{-1}\right)\right],
\end{aligned}
$$

with $f(\tau)$ as defined in eq. (D.3) and

$$
g(\tau)=\left\{\begin{array}{ll}
\sqrt{\tau^{-1}-1} \arcsin ^{2} \sqrt{\tau} & \tau \leq 1 \\
\frac{\sqrt{1-\tau^{-1}}}{2}\left[\log \frac{1+\sqrt{1-\tau^{-1}}}{1-\sqrt{1-\tau^{-1}}}-i \pi\right] & \tau>1
\end{array} .\right.
$$

The expression for the $h \rightarrow Z \gamma$ amplitude, keeping only the fermionic and the $W$ contributions, is:

$$
\begin{aligned}
\frac{\mathcal{A}(h \rightarrow Z \gamma)}{v_{\mathrm{SM}}} \propto\left(\frac{g_{W}^{2} v_{\mathrm{SM}}}{4 m_{W}^{2}}\right) g_{W^{+} W^{-} Z} \times A_{1}\left(m_{h}^{2} / 4 m_{W}^{2}, m_{Z}^{2} / 4 m_{W}^{2}\right) & \\
& +\sum_{\psi=t_{L}, t_{R}, b_{L}, b_{R}} \sum_{i j} 4 N_{c} Q_{\psi} \lambda_{\psi, i j}^{h} \lambda_{\psi, j i}^{Z} F\left(m_{i}, m_{j}, m_{h}, m_{Z}\right),
\end{aligned}
$$

where $g_{W}^{2}$ was defined above and $g_{W^{+} W^{-} Z}$ is the coupling of the $Z$ to a $W^{+} W^{-}$pair in the given model (in the SM one has $g_{W^{+} W^{-} Z}=g c_{W}$ ). It is obtained by projecting the appropriate mass eigenstates after diagonalization of the full system, and therefore includes the effects of mixing with the heavy spin-1 resonances. For the fermionic contribution: $\lambda_{\psi, i j}^{h}$ and $\lambda_{\psi, j i}^{Z}$ are the couplings to the Higgs and $Z$ of the fermion mass eigenstates $i$ and $j$ (see conventions in eq. (F.1) of ref. [37]), with masses $m_{i}$ and $m_{j}$. These are obtained numerically by writing the corresponding coupling matrices in the mass eigenbasis. The function $F\left(m_{i}, m_{j}, m_{h}, m_{Z}\right)$ is given in eq. (F.3) of ref. [37], and in the limit $m_{1}=m_{2} \equiv m$, reduces to $(1 / 2 m) A_{1 / 2}\left(m_{h}^{2} / 4 m^{2}, m_{Z}^{2} / 4 m^{2}\right)$, where $A_{1 / 2}(\tau, \lambda)$ is the standard fermionic loop function for this process (see e.g. [58]):

$$
A_{1 / 2}(\tau, \lambda)=I_{1}(\tau, \lambda)-I_{2}(\tau, \lambda) .
$$

Using the same normalization as above, we have $\mathcal{A}(h \rightarrow Z \gamma)^{\mathrm{SM}} \propto$ $g c_{W} A_{1}\left(m_{h}^{2} / 4 m_{W}^{2}, m_{Z}^{2} / 4 m_{W}^{2}\right)+\sum_{i=t, b} 2 N_{c} Q_{i}\left(g / c_{W}\right)\left(\frac{1}{2}-2 Q_{i} s_{W}^{2}\right) A_{1 / 2}\left(m_{h}^{2} / 4 m_{i}^{2}, m_{Z}^{2} / 4 m_{i}^{2}\right)$.

Open Access. This article is distributed under the terms of the Creative Commons Attribution License (CC-BY 4.0), which permits any use, distribution and reproduction in any medium, provided the original author(s) and source are credited.

\section{References}

[1] ATLAS collaboration, Observation of a new particle in the search for the standard model Higgs boson with the ATLAS detector at the LHC, Phys. Lett. B 716 (2012) 1 [arXiv: 1207.7214] [INSPIRE]. 
[2] CMS collaboration, Observation of a new boson at a mass of $125 \mathrm{GeV}$ with the CMS experiment at the LHC, Phys. Lett. B 716 (2012) 30 [arXiv:1207.7235] [INSPIRE].

[3] D.B. Kaplan and H. Georgi, $\mathrm{SU}(2) \times \mathrm{U}(1)$ breaking by vacuum misalignment, Phys. Lett. B 136 (1984) 183 [INSPIRE].

[4] D.B. Kaplan and H. Georgi, Composite Higgs scalars, Phys. Lett. B 136 (1984) 187 [INSPIRE].

[5] H. Georgi, D.B. Kaplan and P. Galison, Calculation of the composite Higgs mass, Phys. Lett. B 143 (1984) 152 [INSPIRE].

[6] H. Georgi and D.B. Kaplan, Composite Higgs and custodial SU(2), Phys. Lett. B 145 (1984) 216 [INSPIRE].

[7] M.J. Dugan, H. Georgi and D.B. Kaplan, Anatomy of a composite Higgs model, Nucl. Phys. B 254 (1985) 299 [INSPIRE].

[8] R. Contino, Y. Nomura and A. Pomarol, Higgs as a holographic pseudo-Goldstone boson, Nucl. Phys. B 671 (2003) 148 [hep-ph/0306259] [INSPIRE].

[9] G.F. Giudice, C. Grojean, A. Pomarol and R. Rattazzi, The strongly-interacting light Higgs, JHEP 06 (2007) 045 [hep-ph/0703164] [INSPIRE].

[10] M. Gillioz, R. Grober, C. Grojean, M. Muhlleitner and E. Salvioni, Higgs low-energy theorem (and its corrections) in composite models, JHEP 10 (2012) 004 [arXiv:1206.7120] [INSPIRE].

[11] T. Corbett, O.J.P. Eboli, J. Gonzalez-Fraile and M.C. Gonzalez-Garcia, Constraining anomalous Higgs interactions, Phys. Rev. D 86 (2012) 075013 [arXiv:1207.1344] [InSPIRE].

[12] D. Carmi, A. Falkowski, E. Kuflik, T. Volansky and J. Zupan, Higgs after the discovery: a status report, JHEP 10 (2012) 196 [arXiv:1207.1718] [INSPIRE].

[13] E.E. Jenkins, A.V. Manohar and M. Trott, Renormalization group evolution of the standard model dimension six operators I: formalism and $\lambda$ dependence, JHEP 10 (2013) 087 [arXiv: 1308.2627] [INSPIRE].

[14] R. Alonso, M.B. Gavela, L. Merlo, S. Rigolin and J. Yepes, The effective chiral lagrangian for a light dynamical "Higgs particle", Phys. Lett. B $\mathbf{7 2 2}$ (2013) 330 [Erratum ibid. B 726 (2013) 926] [arXiv: 1212.3305] [INSPIRE].

[15] G. Buchalla, O. Catá and C. Krause, Complete electroweak chiral lagrangian with a light Higgs at NLO, Nucl. Phys. B 880 (2014) 552 [arXiv:1307.5017] [InSPIRE].

[16] I. Brivio et al., Disentangling a dynamical Higgs, JHEP 03 (2014) 024 [arXiv:1311.1823] [INSPIRE].

[17] B.A. Dobrescu, Minimal composite Higgs model with light bosons, Phys. Rev. D 63 (2001) 015004 [hep-ph/9908391] [INSPIRE].

[18] K. Agashe, R. Contino and A. Pomarol, The minimal composite Higgs model, Nucl. Phys. B 719 (2005) 165 [hep-ph/0412089] [INSPIRE].

[19] A. Falkowski, Pseudo-goldstone Higgs production via gluon fusion, Phys. Rev. D 77 (2008) 055018 [arXiv: 0711.0828] [INSPIRE].

[20] I. Low, R. Rattazzi and A. Vichi, Theoretical constraints on the Higgs effective couplings, JHEP 04 (2010) 126 [arXiv:0907.5413] [INSPIRE]. 
[21] M. Montull, F. Riva, E. Salvioni and R. Torre, Higgs couplings in composite models, Phys. Rev. D 88 (2013) 095006 [arXiv: 1308.0559] [INSPIRE].

[22] M. Gillioz, R. Grober, A. Kapuvari and M. Muhlleitner, Vector-like bottom quarks in composite Higgs models, JHEP 03 (2014) 037 [arXiv:1311.4453] [INSPIRE].

[23] H.-C. Cheng, B.A. Dobrescu and J. Gu, Higgs mass from compositeness at a multi-TeV scale, arXiv:1311.5928 [INSPIRE].

[24] J. Barnard, T. Gherghetta and T.S. Ray, UV descriptions of composite Higgs models without elementary scalars, JHEP 02 (2014) 002 [arXiv: 1311.6562] [INSPIRE].

[25] R. Contino and A. Pomarol, Holography for fermions, JHEP 11 (2004) 058 [hep-th/0406257] [INSPIRE].

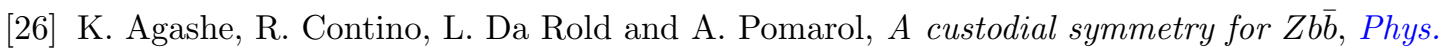
Lett. B 641 (2006) 62 [hep-ph/0605341] [INSPIRE].

[27] R. Contino, T. Kramer, M. Son and R. Sundrum, Warped/composite phenomenology simplified, JHEP 05 (2007) 074 [hep-ph/0612180] [INSPIRE].

[28] S. De Curtis, M. Redi and A. Tesi, The 4D composite Higgs, JHEP 04 (2012) 042 [arXiv:1110.1613] [INSPIRE].

[29] G. Panico and A. Wulzer, The discrete composite Higgs model, JHEP 09 (2011) 135 [arXiv:1106.2719] [INSPIRE].

[30] M.S. Carena, E. Ponton, J. Santiago and C.E.M. Wagner, Light kaluza klein states in Randall-Sundrum models with custodial SU(2), Nucl. Phys. B 759 (2006) 202 [hep-ph/0607106] [INSPIRE].

[31] M.S. Carena, E. Ponton, J. Santiago and C.E.M. Wagner, Electroweak constraints on warped models with custodial symmetry, Phys. Rev. D 76 (2007) 035006 [hep-ph/0701055] [INSPIRE].

[32] C. Csáki, A. Falkowski and A. Weiler, The flavor of the composite pseudo-Goldstone higgs, JHEP 09 (2008) 008 [arXiv:0804.1954] [INSPIRE].

[33] A. Pomarol and F. Riva, The composite Higgs and light resonance connection, JHEP 08 (2012) 135 [arXiv:1205.6434] [INSPIRE].

[34] D. Pappadopulo, A. Thamm and R. Torre, A minimally tuned composite Higgs model from an extra dimension, JHEP 07 (2013) 058 [arXiv: 1303.3062] [INSPIRE].

[35] I. Low and A. Vichi, On the production of a composite Higgs boson, Phys. Rev. D 84 (2011) 045019 [arXiv: 1010.2753] [INSPIRE].

[36] A. Azatov and J. Galloway, Light custodians and Higgs physics in composite models, Phys. Rev. D 85 (2012) 055013 [arXiv:1110.5646] [INSPIRE].

[37] A. Azatov, R. Contino, A. Di Iura and J. Galloway, New prospects for Higgs compositeness in $h \rightarrow Z \gamma$, Phys. Rev. D 88 (2013) 075019 [arXiv: 1308.2676] [INSPIRE].

[38] J. Mrazek, A. Pomarol, R. Rattazzi, M. Redi, J. Serra et al., The other natural two Higgs doublet model, Nucl. Phys. B 853 (2011) 1 [arXiv:1105.5403] [INSPIRE].

[39] J. Elias-Miro, J.R. Espinosa, E. Masso and A. Pomarol, Renormalization of dimension-six operators relevant for the Higgs decays $h \rightarrow \gamma \gamma, \gamma Z$, JHEP 08 (2013) 033 [arXiv:1302.5661] [INSPIRE]. 
[40] J. Elias-Miro, J.R. Espinosa, E. Masso and A. Pomarol, Higgs windows to new physics through $D=6$ operators: constraints and one-loop anomalous dimensions, JHEP 11 (2013) 066 [arXiv: 1308.1879] [INSPIRE].

[41] A. Pomarol and F. Riva, Towards the ultimate SM fit to close in on Higgs physics, JHEP 01 (2014) 151 [arXiv:1308.2803] [INSPIRE].

[42] ATLAS collaboration, Combined measurements of the mass and signal strength of the Higgs-like boson with the ATLAS detector using up to $25 \mathrm{fb}^{-1}$ of proton-proton collision data, ATLAS-CONF-2013-014 (2013).

[43] M. Redi and A. Weiler, Flavor and CP invariant composite Higgs models, JHEP 11 (2011) 108 [arXiv: 1106.6357] [INSPIRE].

[44] L. Da Rold, C. Delaunay, C. Grojean and G. Perez, Up asymmetries from exhilarated composite flavor structures, JHEP 02 (2013) 149 [arXiv:1208.1499] [INSPIRE].

[45] M. Redi, V. Sanz, M. de Vries and A. Weiler, Strong signatures of right-handed compositeness, JHEP 08 (2013) 008 [arXiv: 1305.3818] [INSPIRE].

[46] C. Delaunay et al., Light non-degenerate composite partners at the LHC, JHEP 02 (2014) 055 [arXiv: 1311.2072] [INSPIRE].

[47] A. Pomarol and J. Serra, Top quark compositeness: feasibility and implications, Phys. Rev. D 78 (2008) 074026 [arXiv:0806.3247] [INSPIRE].

[48] L. Da Rold, Solving the $A_{F B}^{b}$ anomaly in natural composite models, JHEP 02 (2011) 034 [arXiv: 1009.2392] [INSPIRE].

[49] ATLAS collaboration, An update of combined measurements of the new Higgs-like boson with high mass resolution channels, ATLAS-CONF-2012-170 (2012).

[50] ATLAS collaboration, Combined coupling measurements of the Higgs-like boson with the ATLAS detector using up to $25 \mathrm{fb}^{-1}$ of proton-proton collision data, ATLAS-CONF-2013-034 (2013).

[51] ATLAS collaboration, Evidence for Higgs boson decays to the $\tau^{+} \tau^{-}$final state with the ATLAS detector, ATLAS-CONF-2013-108 (2013).

[52] CMS collaboration, Search for the Standard-Model Higgs boson decaying to tau pairs in proton-proton collisions at $\sqrt{s}=7$ and 8 TeV, CMS-PAS-HIG-13-004 (2013).

[53] C. Anastasiou, E. Furlan and J. Santiago, Realistic composite Higgs models, Phys. Rev. D 79 (2009) 075003 [arXiv:0901.2117] [INSPIRE].

[54] G. Panico, M. Redi, A. Tesi and A. Wulzer, On the tuning and the mass of the composite Higgs, JHEP 03 (2013) 051 [arXiv: 1210.7114] [INSPIRE].

[55] R. Contino, L. Da Rold and A. Pomarol, Light custodians in natural composite Higgs models, Phys. Rev. D 75 (2007) 055014 [hep-ph/0612048] [InSPIRE].

[56] R. Barbieri and G.F. Giudice, Upper bounds on supersymmetric particle masses, Nucl. Phys. B 306 (1988) 63 [inSPIRE].

[57] G.W. Anderson and D.J. Castano, Measures of fine tuning, Phys. Lett. B 347 (1995) 300 [hep-ph/9409419] [INSPIRE].

[58] A. Djouadi, The anatomy of electro-weak symmetry breaking. I: the Higgs boson in the standard model, Phys. Rept. 457 (2008) 1 [hep-ph/0503172] [INSPIRE]. 
[59] A. Djouadi, V. Driesen, W. Hollik and A. Kraft, The Higgs photon $-Z$ boson coupling revisited, Eur. Phys. J. C 1 (1998) 163 [hep-ph/9701342] [INSPIRE].

[60] T. Hahn and M. Pérez-Victoria, Automatized one loop calculations in four-dimensions and D-dimensions, Comput. Phys. Commun. 118 (1999) 153 [hep-ph/9807565] [InSPIRE]. 